\title{
A UNIFIED MODEL OF THE FERMI BUBBLES, MICROWAVE HAZE, AND POLARIZED RADIO LOBES: REVERSE SHOCKS IN THE GALACTIC CENTER'S GIANT OUTFLOWS
}

\author{
Roland M. Crocker ${ }^{1,5}$, Geoffrey V. Bicknell ${ }^{1}$, Andrew M. Taylor ${ }^{2,6}$, and Ettore Carretti ${ }^{3,4}$ \\ Research School of Astronomy and Astrophysics, Australian National University, Canberra, ACT, Australia \\ ${ }^{2}$ Dublin Institute of Advanced Studies, Dublin, Ireland \\ ${ }^{3}$ INAF-Osservatorio di Cagliari, Via della Scienza 5, I-09047 Selargius, Italy \\ ${ }^{4}$ CSIRO Astronomy \& Space Science, Marsfield, N.S.W., Australia \\ Received 2015 April 17; accepted 2015 June 16; published 2015 July 27
}

\begin{abstract}
The Galactic center's giant outflows are manifest in three different, nonthermal phenomena: (1) the hard-spectrum, $\gamma$-ray "Fermi bubbles" emanating from the nucleus and extending to $|b| \sim 50^{\circ}$; (2) the hard-spectrum, totalintensity microwave ( $20-40 \mathrm{GHz})$ "haze" extending to $|b| \sim 35^{\circ}$ in the lower reaches of the Fermi bubbles; and (3) the steep-spectrum, polarized, "S-PASS" radio $(\sim 2-20 \mathrm{GHz})$ lobes that envelop the bubbles and extend to $|b| \sim 60^{\circ}$. We find that the nuclear outflows inflate a genuine bubble in each Galactic hemisphere that has the classical structure, working outward, of reverse shock, contact discontinuity (CD), and forward shock. Expanding into the finite pressure of the halo and given appreciable cooling and gravitational losses, the CD of each bubble is now expanding only very slowly. We find observational signatures in both hemispheres of giant, reverse shocks at heights of $\sim 1 \mathrm{kpc}$ above the nucleus; their presence ultimately explains all three of the nonthermal phenomena mentioned above. Synchrotron emission from shock-reaccelerated cosmic-ray electrons explains the spectrum, morphology, and vertical extent of the microwave haze and the polarized radio lobes. Collisions between shockreaccelerated hadrons and denser gas in cooling condensations that form inside the $\mathrm{CD}$ account for most of the bubbles' $\gamma$-ray emissivity. Inverse Compton emission from primary electrons contributes at the $10 \%-30 \%$ level. Our model suggests that the bubbles are signatures of a comparatively weak but sustained nuclear outflow driven by Galactic center star formation over $\gtrsim$ few $\times 10^{8} \mathrm{yr}$.
\end{abstract}

Key words: cosmic rays - gamma rays: diffuse background

\section{INTRODUCTION}

\subsection{Overview}

The Fermi bubbles are enigmatic, giant $\gamma$-ray structures emanating north and south from the nucleus of the Milky Way (Dobler et al. 2010; Su et al. 2010; Su \& Finkbeiner 2012; Ackermann et al. 2014; Yang et al. 2014) and extending to heights above the Galactic plane approaching $8 \mathrm{kpc}$. Features coincident with the bubbles or some component of the bubbles have been discovered in total-intensity radio and microwave emission, polarized radio and microwave emission, and X-ray emission (Bland-Hawthorn \& Cohen 2003; Finkbeiner 2004; Dobler \& Finkbeiner 2008; Dobler 2012a; Ade et al. 2013; Carretti et al. 2013; Kataoka et al. 2013; Tahara et al. 2015).

The energy source of the bubbles remains in contention, as does the $\gamma$-ray emission process that illuminates them. Broadly speaking, the bubbles might be the signatures of a fairly recent (within a $\sim$ Myr timescale) explosive outburst (Su et al. 2010; Mertsch \& Sarkar 2011; Zubovas et al. 2011; Guo \& Mathews 2012; Yang et al. 2012; Zubovas \& Nayakshin 2012; Fujita et al. 2013; Barkov \& Bosch-Ramon 2014; Mou et al. 2014; Wardle \& Yusef-Zadeh 2014) from the central supermassive black hole with their $\gamma$-ray emission originating in inverse Compton (IC) collisions of a nonthermal electron population accelerated in association with this activity. Alternatively, the bubbles may result from the integrated effect of more or less secular processes associated with the inner Galaxy (Thoudam 2013) or Galactic nucleus, e.g., tidal disruption events that occur there every $10^{4}-10^{5} \mathrm{yr}$ (Cheng

\footnotetext{
5 Future Fellow.

${ }^{6}$ Schrödinger Fellow.
}

et al. 2011, 2015) or the concentrated and sustained star formation activity (e.g., Lacki 2014) that occurs throughout the nuclear region (inner 200-300 pc diameter region around the black hole).

Invoking sustained nuclear processes as the origin of the bubbles complements the possibility that their $\gamma$-ray emission originates not from IC emission but from hadronic collisions experienced by a population of cosmic-ray protons and heavier ions (Crocker \& Aharonian 2011; Crocker 2012; Crocker et al. 2014). Such particles lose energy much more slowly than the high-energy electrons requisite for IC $\gamma$-ray production, thereby allowing the total current energy content of the bubbles to be accumulated more gradually from relatively less powerful processes such as star formation (the Eddington luminosity of the Galaxy's 4 million solar mass black hole is $5 \times 10^{44} \mathrm{erg} \mathrm{s}^{-1}$; in contrast, the Milky Way's nuclear star formation releases a mechanical power of $\sim 3 \times 10^{40} \mathrm{erg} \mathrm{s}^{-1}$; see below).

At present, there are strengths and weaknesses to either of these broad scenarios. IC scenarios share the attractive feature that the very same electron population that putatively supplies the IC $\gamma$-rays can, for magnetic field strengths approaching $10 \mu \mathrm{G}$, also supply the hard microwave haze emission via synchrotron radiation (Dobler et al. 2010; Su et al. 2010; Ackermann et al. 2014). On the other hand, if described by a single power law, synchrotron emission from this population of electrons would not explain the comparatively steep radio spectrum measured between 2.3 and $23 \mathrm{GHz}$ for the bubbles' polarized, radio lobe counterparts (Carretti et al. 2013). Furthermore, the fact that the $\gamma$-ray spectrum of the bubbles does not steepen (Ackermann et al. 2014)—rather, it seems to harden (Selig et al. 2014; Yang et al. 2014)—with increasing 
Galactic latitude is difficult to explain naturally within IC scenarios given the attenuation of the optical and infrared background relative to the cosmic microwave background $(\mathrm{CMB})$ as the distance from the Galaxy increases.

Another apparent weakness of many explosive, active galactic nucleus (AGN) related scenarios is that they require that we are viewing the bubbles at a privileged time at most a few million years since the outburst that created them, with the bubbles currently undergoing rapid expansion at speeds $\gtrsim 2000 \mathrm{~km} \mathrm{~s}^{-1}$. Rapid expansion is required in order either to transport the $\gtrsim \mathrm{TeV}$ electrons (required to produce $\gtrsim \mathrm{GeV} \gamma$ rays via IC on the CMB) from the nucleus to the bubbles' peripheries within their $\lesssim 10^{6} \mathrm{yr}$ loss times (in which case speeds $\gtrsim 7000 \mathrm{~km} \mathrm{~s}^{-1} \simeq 0.02 \times c$ are actually required) or to have sufficiently energetic shocks at the expanding edges of the bubbles that cascading turbulence injected there may energize similarly energetic electrons in situ (Mertsch \& Sarkar 2011). Yet X-ray observations of the bubbles have only indicated weak features associated with the bubble edges (Su et al. 2010; Kataoka et al. 2013; Fang \& Jiang 2014; Tahara et al. 2015), implying rather mild expansion speeds, $\lesssim 300 \mathrm{~km} \mathrm{~s}^{-1}$ (close to the sound speed). Closer to the base of the bubbles, data obtained in UV absorption observations by Fox et al. (2014) along the $(l, b)=(10.4,+11.2)$ sightline (to quasar PDS 456) reveal cool $\left(\sim 10^{4} \mathrm{~K}\right)$ gas entrained into a biconical, $\sim 900 \mathrm{~km} \mathrm{~s}^{-1}$ nuclear outflow 2.5-4.0 Myr ago.

Moreover, there are other indications that the bubbles are rather long-lived structures.

1. The fact that both bubbles lean away from the $l=0$ meridian toward Galactic west and, moreover, there are extended plumes of polarized, $2.3 \mathrm{GHz}$ emission leaving northwest (south west) from the high-latitude regions of the north (south) bubble (Carretti et al. 2013) suggests that the structures are distorted by a pressure gradient within the Galactic halo set up by the motion of the Milky Way through the local medium toward Andromeda. The $\sim 50 \mathrm{~km} \mathrm{~s}^{-1}$ relative speed (van der Marel et al. 2012) of this motion implies a rather gentle effect that requires long timescales/low bubble gas velocities to take effect, consistent with the $\sim 100-150 \mathrm{~km} \mathrm{~s}^{-1}$ flow velocities we determine below for gas in the bubbles (see Yang et al. 2012, where it was shown that an unreasonably large speed of the Galaxy through the local medium was required to produce the bubbles' distortion in the case that they are expanding rapidly owing to AGN activity).

2. The $\sim 100 \mathrm{~km} \mathrm{~s}^{-1}$ rotation of the bubbles' waist in the plane is apparently inscribed into giant radio ridges on the near surface of the bubbles (Carretti et al. 2013), again requiring that the structures not grow too quickly (here meaning $\left.\lesssim 10^{7} \mathrm{yr}\right)$.

3. The steep spectrum of the bubbles' polarized radio counterparts between 2.3 and $23 \mathrm{GHz}$ suggests that electrons emitting in this frequency range have had time to cool, mix, and accumulate; this requires $\gtrsim 3 \times 10^{7} \mathrm{yr}$ for a reasonable magnetic field amplitude.

4. Finally, observations of UV absorption features toward background quasar and AGB stars have established the existence of individual parcels of ionized gas at low Galactic longitude but varying latitude (Keeney et al. 2006; Zech et al. 2008). Kinematic analysis points to this gas participating in a nuclear fountain flow. Individual gas parcels seem to have been launched from the nucleus at different times in the past, ranging from 20-50 Myr to more than 800 Myr. The parcels are all now either rising to or falling from a maximum height of $\sim 12 \mathrm{kpc}$, similar to the vertical extent of the bubbles. Some of this material (Zech et al. 2008) appears significantly supersolar in metallicity. This phenomenology points to the existence of a long-standing, starformation-driven, nuclear fountain; as we expand on later in the paper, we believe that this material is condensing in the shell (R3 in Figure 1) leading up to the contact discontinuity (CD) in each bubble.

Turning now to the star-formation-driven hadronic model (Crocker \& Aharonian 2011; Crocker 2012; Crocker et al. 2014), this has the appealing feature that the power currently being delivered into cosmic-ray ions by nuclear star formation is elegantly sufficient to explain the bubbles' (putatively hadronic) $\gamma$-ray luminosity in steady state. On the other hand, in the original instantiation of this scenario, the low density of target gas nuclei in the bubble plasma implied a very long proton-proton loss time of $\mathrm{a} \gtrsim$ few $\mathrm{Gyr}$, this being the timescale required to establish a steady state in the first place (i.e., approximately the timescale required to accumulate a sufficient total energy in cosmic-ray ions in the bubbles to power their instantaneous $\gamma$-ray luminosity given the low gas density). Some of us have recently shown, however, that accumulation of hadronic cosmic rays into gas condensations formed by local thermal instability in the bubbles can significantly reduce the timescale associated with the hadronic scenario to $\sim$ few $\times$ $10^{8} \mathrm{yr}$ (Crocker et al. 2014). Another point concerning the hadronic scenario is that although secondary electrons (and positrons) are a natural concomitant of hadronic $\gamma$-ray production, it is difficult to explain the spectrum of the microwave haze with such secondaries since IC and synchrotron losses would be expected, in a single-zone model, to steepen the steady-state secondary distribution such that it is inconsistent with the hard haze emission (e.g., Ackermann et al. 2014; Cheng et al. 2014b). It seems, then, that even the hadronic scenario requires primary electrons at some level to explain the totality of giant outflow phenomenology.

Indeed, the power in cosmic-ray primary electrons accelerated in concert with nuclear star formation is a good match to the bubbles' $2.3 \mathrm{GHz}$ synchrotron luminosity (Carretti et al. 2013). The complement to these points is that the nucleus is underluminous in both diffuse $\gamma$-ray and radio continuum emission given how much star formation occurs there (Crocker et al. 2011a, 2011b; Crocker 2012), and this seems to be because both cosmic-ray electrons and ions are advected out of the region in the large-scale outflow before they can radiate. Thus, these particles radiate on the size scale of the bubbles into which the nuclear outflow delivers them. As we show below, however, the passage from nucleus to bubbles is not a smooth journey for these particles.

\subsection{Outline of Model}

Here we briefly explain our model for the bubbles' structure and nonthermal emission phenomenology. A schematic showing the configuration of the northern bubble according to our model is shown in Figure 1 for reference.

1. Co-entrained hot plasma, magnetic fields, and cosmic rays are energized within the central $\sim 100 \mathrm{pc}$ (in radius) region of the Galactic nucleus. The pressure gradient 


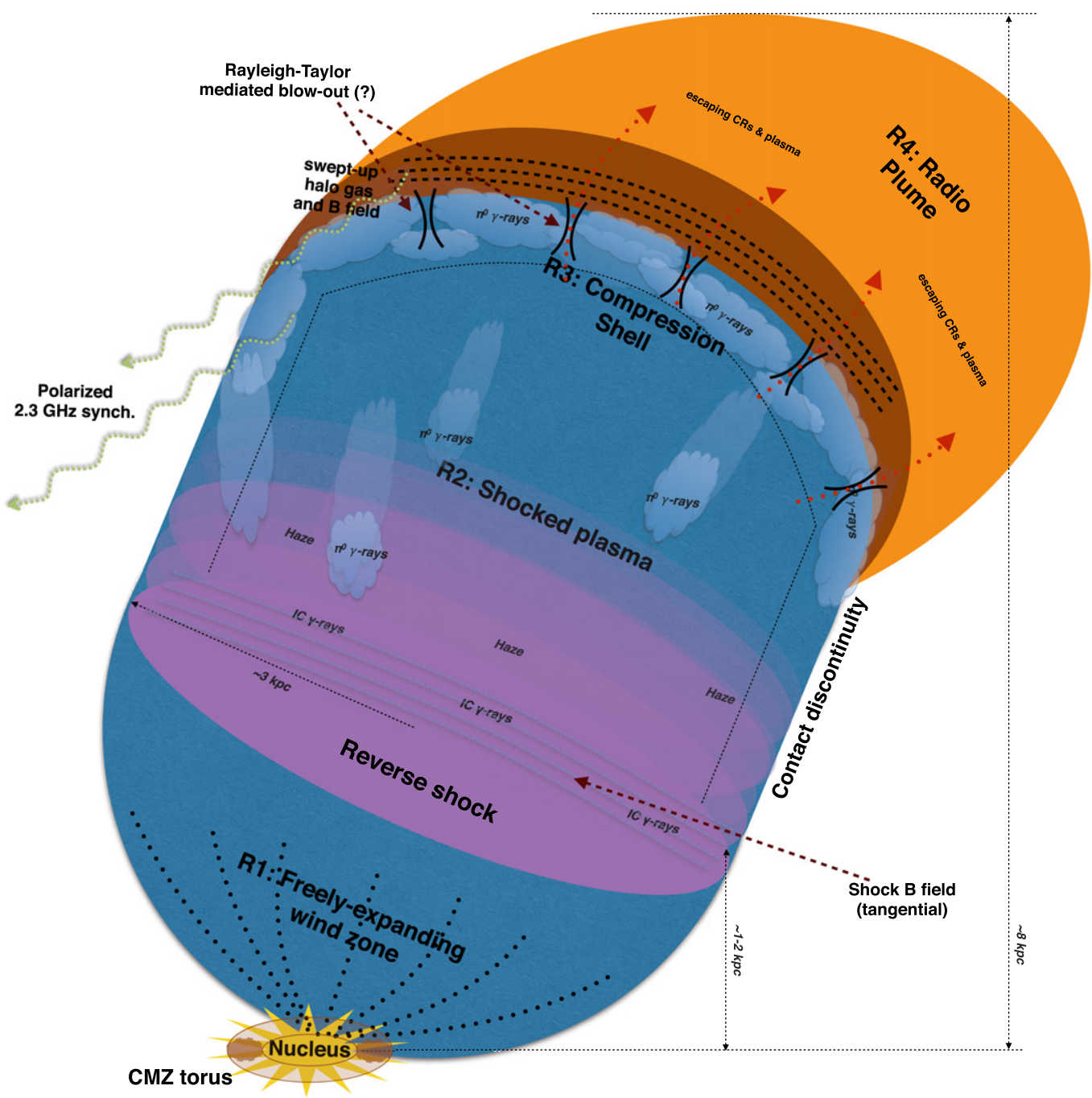

Figure 1. Schematic showing the main features of the north bubble according to our model.

supplied by these interstellar medium (ISM) phases (with the hot plasma dynamically dominant) accelerates a nuclear wind that quickly reaches $\gtrsim 500 \mathrm{~km} \mathrm{~s}^{-1}$. The dense and massive molecular gas torus circumscribing the injection region collimates this outflow and directs it perpendicular to the plane. Shocks driven into the hot ISM, which is more dense at low latitudes, may account for the X-ray edges observed by Bland-Hawthorn \& Cohen (2003). Radiative shocks driven into atomic and/ or molecular gas may also contribute.

2. The bubbles are inflated by the injection of hot plasma in the nuclear outflow over timescales of at least a few $x$ $10^{8} \mathrm{yr}$. Characteristic scales for the mean mechanical power and mass flux into the bases of the bubbles are $\sim 10^{40} \mathrm{erg} \mathrm{s}^{-1}$ and $\sim 0.1 M_{\odot} \mathrm{yr}^{-1}$, respectively.

3. This outflow, however, is not powerful enough-given the strong gravitational field, the thermal pressure of the overlying halo gas, and the occurrence of gas cooling - to break out of the Galaxy.

4. Instead, the outflow generates the two Fermi bubbles and associated phenomena. The bubbles inflate against an atmosphere of non-negligible pressure. An important ingredient of our scenario is that recent work indicates that the gas density and pressure in the halo, especially in the vicinity of the bubbles, are larger than has often been assumed in the past (e.g., Stocke et al. 2013; Miller \& Bregman 2014).

5. The bubbles develop the classical wind-blown bubble structure of (starting from the smallest scales and working outward) (i) a reverse shock, (ii) a CD, and (iii) a forward shock; see Figure 3.

6. The CD separates ISM material swept up and shocked in the downstream of the forward shock from shocked bubble material in the downstream of the internal reverse shock. (In our model, the $\gamma$-ray edge of the bubbles is identified with the CD. $)^{8}$

\footnotetext{
7 A further consideration is that it is increasingly likely that the gas around the bubbles is itself in a flow driven into the halo by star formation occurring in the Milky Way's molecular ring (at galactocentric radius of $\sim 4 \mathrm{kpc}$ ) and/or the bar (Breitschwerdt et al. 1991, 2002; Everett et al. 2008, 2010; Kretschmer et al. 2013; de Boer \& Weber 2014); halo density models that assume hydrostatic equilibrium may not convey the full story in the inner Galaxy.

8 We tentatively identify some of the $\gamma$-ray substructure in the base of the bubbles—e.g., the cocoon (Su \& Finkbeiner 2012; Ackermann et al. 2014) —as IC emission from upstream cosmic-ray electrons interacting with the dense photon fields close to the disk before reaching the shocks and from downstream electrons that have been reaccelerated upon reaching one of the shocks (but are not able to reach very far past them).
} 
7. The bubbles quickly (within $\sim 10^{7} \mathrm{yr}$ ) reach pressure equilibrium with their environment; at this point (neglecting, as we do in our current model, cooling in the region between forward shock and CD) the forward shock degenerates into a sound wave. The CD decelerates even faster and is subsonic after only $\lesssim 10^{6} \mathrm{yr}$; at present it is expanding at only $\sim$ few $\mathrm{km} \mathrm{s}^{-1}$.

8. Thermal plasma and cosmic rays initially suffer adiabatic and radiative energy losses as they are blown out of the nucleus on the conically expanding outflow (R1 in Figure 1). However, they encounter giant reverse shocks (one at $\sim 1 \mathrm{kpc}$ height in each bubble) where the ram pressure of this flow equilibrates with the thermal pressure of the shocked, downstream gas (contrast the model of Lacki 2014, where the ram pressure of the expanding outflow equilibrates with the thermal pressure of the background Galactic halo atmosphere). At these shocks, the plasma and cosmic rays are reheated/ reaccelerated. (In the remainder of this paper, unless otherwise noted, the shocks we refer to generically are these reverse shocks interior to the $\mathrm{CD}$.)

9. In the immediate downstream of the shocks, there is a systematic subsonic flow of $v_{\text {down }} \sim 1 / 4 v_{\mathrm{w}} \sim$ $100-150 \mathrm{~km} \mathrm{~s}^{-1}$ (with $v_{\mathrm{w}}$ the wind speed immediately upstream of the shock). There is energy-independent transport of the nonthermal particles into the bubbles mediated by this flow in combination with cosmic-ray streaming at approximately the Alfvén speed. Highenergy cosmic-ray electrons produce IC and synchrotron emission on photon and magnetic fields in the shock downstream. Given that there is a systematic flow (such that, at any particular distance from the shock, all electrons are the same "age"), the spectrum of these electrons (and their resultant emission) is hard (mirroring the injection distribution) up to some time-dependen$\mathrm{t}$ (and thus distance-dependent) maximal energy above which the spectrum is exponentially suppressed. Given the rather slow transport speeds and relatively short cooling times, microwave-synchrotron-emitting electrons reach only $\sim 1-2 \mathrm{kpc}$ past the shocks and $\gtrsim \mathrm{GeV}$ ICemitting electrons only reach a few $\times 10^{2} \mathrm{pc}$. (In contrast, cosmic-ray protons and $2.3 \mathrm{GHz}$ synchrotron-emitting electrons survive to reach the full extent of the bubbles.) Altogether, we show below that the hard-spectrum synchrotron radiation from cosmic-ray electrons reaccelerated on the shocks and transported downstream provides an excellent match to the luminosity, spectrum, distribution, and morphology of the microwave haze.

10. As the bubbles age and their volume slowly increases (more than compensating for their density decline), the radiative losses of the plasma gradually become more significant. In fact, there is a steady-state limit (at constant radius and mass) where the bubbles reach pressure equilibrium with the environment and the cooling radiation from their interior plasma completely saturates the injected mechanical energy. As this limit is approached, an increasingly large fraction of the hot plasma mass injected into the bubbles is lost by dropout as cooling leads to local thermal instability that causes individual parcels of gas to cool, collapse, lose buoyancy, and fall out of the bubbles under gravity.
11. Because of the density-squared dependence of cooling, this mass dropout process dominantly occurs in the somewhat overdense shell of material that forms inside of the $\mathrm{CD}$, where the slow flow of plasma away from the reverse shock is gradually arrested (R3 in Figure 1).

12. While the plasma evolves radiatively in the shell (as it cools down into localized, overdense condensations), cosmic-ray hadrons and magnetic fields, in constrast, continue to evolve adiabatically. Their compression in the shell material leads to adiabatic energy gain; they are compressed until they equilibrate with the thermal pressure of the volume-filling plasma in $\mathrm{R} 2$ between the shell and the reverse shock. In other words, adiabatic evolution of these nonthermal phases leads them to become the dominant dynamic agent in the shell.

13. The ultimate extent and mass of shell R3 are determined by the pressure balance condition explained above and the further condition that the shell's cooling radiation saturates the enthalpy flux represented by the slow flow of hot plasma into it from R2.

14. Given that both the mass of shell target material and the hadronic cosmic-ray energy density in the shell can be determined from these considerations, we can calculate the hadronic emission from the shell; we find that this essentially saturates at the observed $\gamma$-ray luminosity of the bubbles for reasonable parameter values. The fact that the dominant hadronic emission occurs mostly in the shell explains why the volumetric emission from the bubbles peaks toward their edges (as required to reproduce their flat projected surface brightness; $\mathrm{Su}$ et al. 2010).

15. As for the protons and heavier ions, the lower-energy cosmic-ray electrons that live long enough to reach the shell are also adiabatically compressed and reenergized there. At the same time, collisions between the adiabatically compressed hadron population and the shell gas inject secondary electrons (+positrons) into R3. Unlike for the region downstream of the reverse shocks, however, in the shells there is a mixture of electron populations with different ages and thus different characteristic energies. This leads to the integrated, steady-state shell electron spectrum being steepened by the canonical difference in spectral index of 1 associated with synchrotron/IC losses. This cooled primary + locally produced secondary electron population synchrotronradiates on the adiabatically compressed shell magnetic field, explaining the steep-spectrum, polarized emission measured between 2.3 and $23 \mathrm{GHz}$.

16. The shell/CD does not constitute a perfect boundary, however: observationally, in $2.3 \mathrm{GHz}$ polarization data, there are plumes of radio emission that extend northwest and southwest beyond the $\gamma$-ray boundaries of the bubbles (R4). Recent X-ray evidence (Tahara et al. 2015) seems to indicate hot plasma venting from the north bubbles, roughly coincident with the northern radio plume. We speculate that these plumes are symptomatic of the breakout of the light fluid of comixed cosmic rays, plasma, and magnetic fields through the heavy shell, mediated by the Rayleigh-Taylor instability. However, the CD generates the (required) $\gamma$ ray edge because, even if some cosmic-ray protons also escape through this boundary, this delimits the region of 
dense target gas accumulated into the shell from the interior flow.

\subsection{Outline of Paper}

The remainder of this paper is structured as follows. We describe the input data that frame the problem in Section 2. In Section 3 we show that, incorporating the effects of gravity, the bubbles should contain giant, reverse shocks at heights of $\sim 1 \mathrm{kpc}$ into the outflows north and south from the nucleus. In Section 4 we set out a semianalytic calculation describing the evolution of a bubble fed by an outflow of fixed mechanical power and mass flux expanding into an atmosphere of finite, non-negligible pressure. We show that, adopting values for these quantities apposite to the Fermi bubbles, such a bubble reaches the size scale of the Fermi bubbles after a few $\times 10^{8} \mathrm{yr}$ and that the asymptotic radius of such a bubble for these parameters is not much larger than that of the observed Fermi bubbles. This finding removes any "Why now?" problem and justifies our subsequent steady-state treatment of the bubbles' nonthermal emission. In Section 5 we describe our procedure for delimiting the region of the parameter space defined by the energy and mass fluxes ( $\dot{E}_{0}$ and $\dot{M}$, respectively) injected at the nucleus that describe the present-day bubbles. In Section 6 we demonstrate explicitly that, in the small region of parameter space consistent with prior constraints, the bubbles' (i) predicted hadronic $\gamma$-ray emission, (ii) predicted microwave synchrotron emission, and (iii) radio continuum synchrotron emission all match the observed values. In other words, we show that our scenario consistently explains, in a unified model, the nonthermal phenomena described as the "Fermi bubbles," the "microwave haze," and the "S-PASS lobes." In Section 7 we review specific observations that seem to support (or, in one case, may challenge) our model. Finally, in Section 8 we set out implications of our model and future work.

\section{PRELIMINARIES}

\subsection{Geometry}

Adopting a distance to the Galactic center of $8 \mathrm{kpc}$, geometrically the bubbles can be approximated as a pair of spheres with radii $\sim 3.2 \mathrm{kpc}$ with a total volume of $V_{\mathrm{FB}} \simeq$ $8.4 \times 10^{66} \mathrm{~cm}^{3}$ and a combined surface area of $A_{\mathrm{FB}} \simeq$ $2.5 \times 10^{45} \mathrm{~cm}^{2}$. A better characterization of the bubbles' real geometry seems to be that they are bi-cones emanating north and south from the nucleus to heights of $2-3 \mathrm{kpc}$ above the plane, above which they collimate into cylinders (BlandHawthorn \& Cohen 2003; Carretti et al. 2013). The Fermi bubbles reach $\sim 7 \mathrm{kpc}$ maximal height above the Galactic center accounting for projection effects; the polarized radio lobes extend up to $\sim 8 \mathrm{kpc}$. A schematic showing the configuration of the northern bubble according to our model is shown in Figure 1.

\subsection{Other Parameters}

Kataoka et al. (2013) have performed scans across the limbs of both the northern and southern bubbles at high latitudes with the Suzaku X-ray satellite. In neither scan do these authors find evidence for a change of temperature between the exterior and interior plasma; rather, the plasma temperature seems constant at $\sim(3.0-3.5) \times 10^{6} \mathrm{~K}$. For the northern scan, starting in the bright North Polar Spur (NPS) feature (located east in projection from the northern bubble) and scanning inward across the bubble edge, these authors find a drop in emission measure $(\mathrm{EM})$ at the edge from $(5-6) \times 10^{-2} \mathrm{~cm}^{-6} \mathrm{pc}$ outside to (3-4) $\times 10^{-2} \mathrm{~cm}^{-6} \mathrm{pc}$ inside. In the south, the emission measure is approximately constant at $(1-2) \times 10^{-2} \mathrm{~cm}^{-6} \mathrm{pc}$ across the scan. Adopting a total cross-sectional area of $A_{\mathrm{FB}, \mathrm{X}}=7.0 \times 10^{44} \mathrm{~cm}^{2}$, we find an average line-of-sight distance through the bubbles of $l_{\mathrm{LOS}} \equiv V_{\mathrm{FB}} / A_{\mathrm{FB}, \mathrm{X}} \simeq 3.7 \mathrm{kpc}$. With this distance scale and assuming for the moment a uniform density distribution for the bubble plasma, a characteristic ionized hydrogen number density in the bubbles can be estimated as $\left\langle n_{\mathrm{H}^{+}}^{\mathrm{FB}}\right\rangle \sim \sqrt{\mathrm{EM} / l_{\mathrm{LOS}}} \simeq(1-3) \times 10^{-3} \mathrm{~cm}^{-3}$. Using the volumetric density estimate, the total plasma mass of the bubbles is then estimated as

$$
M_{\mathrm{FB}} \simeq 1.4 m_{\mathrm{p}} n_{\mathrm{H}} V_{\mathrm{FB}}=2.1 \times 10^{7} M_{\odot}\left(\frac{\left\langle n_{\mathrm{H}^{+}}^{\mathrm{FB}}\right\rangle}{0.003 \mathrm{~cm}^{-3}}\right)
$$

In reality, we show below that most of the emission measure through the bubbles (and therefore most of their observed thermal X-ray emission) is contributed by gas in a shell whose real density is larger than the volumetric average density estimated above by a factor $\left\langle n_{\mathrm{H}^{+}}^{\text {shell }}\right\rangle /\left\langle n_{\mathrm{H}^{+}}^{\mathrm{FB}}\right\rangle \sim$ $\left(2 \Delta r_{\text {shell }} / l_{\text {LOS }}\right)^{-1 / 2}$, and thickness (conservatively) $r_{\text {shell }} \lesssim$ $2 \mathrm{kpc}$, inside the CD. The fitted X-ray temperature is thus likely to be characteristic of this shell; we expect the volumefilling, shocked plasma between the shell and the reverse shock to be hotter and more rarefied.

Whatever the exact configuration of plasma within the bubbles, consistent with our modeling, they are not strongly overpressured with respect to the surrounding halo (consistent with the absence of obvious X-ray limb brightening in their higher reaches; Kataoka et al. 2013; Tahara et al. 2015). Again working with the characteristic, volumetric-average number density for the moment, the pressure in the bubbles $P_{\mathrm{FB}}=$ $n_{\text {tot }}^{\mathrm{FB}} k_{B} T_{\mathrm{FB}} \simeq 2.0 \mathrm{eV} \mathrm{cm}^{-3} n_{\mathrm{H}}^{\mathrm{FB}} /\left(0.003 \mathrm{~cm}^{-3}\right) T_{\mathrm{FB}} /\left(3.5 \times 10^{6} \mathrm{~K}\right)$ with the total plasma number density $n_{\text {tot }}^{\mathrm{FB}} \simeq 2.3 n_{\mathrm{H}}^{\mathrm{FB}}$ (adopting a universal $n_{\mathrm{He}}=0.1 n_{\mathrm{H}}$ in a totally ionized, electrically neutral plasma and ignoring metals for the moment).

This bubble pressure should be compared with the determination of halo pressures derived from the recent modeling of Miller \& Bregman (2014) of O vIII emission line data. Adopting halo metallicities of $\sim 0.3 Z_{\odot}$ as suggested by independent evidence, these authors determine a halo density profile that gives $n_{e} \simeq 2 \times 10^{-3}$ at a halo height of $z=8 \mathrm{kpc}$ and $n_{e} \simeq 6 \times 10^{-3}$ at $4 \mathrm{kpc}$. Together with their determination of a fairly constant halo gas temperature of $\sim 2 \times 10^{6} \mathrm{~K}$, these results suggest a total atmospheric pressure around the bubbles of $P_{\text {ext }}=n_{\text {tot }}^{\text {halo }} T^{\text {halo }} \sim 2.3 / 1.2 \times\left(6 \times 10^{-3} \mathrm{~cm}^{-3}\right) \times 2 \times 10^{6} \mathrm{~K}=$ $3.2 \times 10^{-12} \mathrm{dyn} \mathrm{cm}^{-2}=2.0 \mathrm{eV} \mathrm{cm}^{-3}$ at their $\sim 4 \mathrm{kpc}$ halfheight.

\subsection{Discussion of Total Energetics and Other Parameters}

In the limit that radiative losses can be neglected and the atmospheric density treated as constant, the total enthalpy of a slowly inflated bubble is

$$
H \equiv \frac{\gamma}{\gamma-1} P_{\mathrm{ext}} V=\dot{E} t
$$


where $\dot{E}$ is the average mechanical power fed into the bubble over time $t$ and $\gamma$ is the effective adiabatic index of the bubble fluid. Employing the atmospheric pressure of $P_{\text {ext }}=2.0 \mathrm{eV} \mathrm{cm}^{-3}$ motivated above, this suggests $H_{\mathrm{FB}} \simeq$ $6.7 \times 10^{55} \mathrm{erg}$ for the bubbles (probably a lower limit as it neglects the $z$-dependence of the halo density and pressure). Accounting only for core-collapse supernovae $(\mathrm{SNe})$, the minimum mechanical power injected by nuclear star formation can be estimated from the Galactic center (GC) star formation rate $\mathrm{SFR}_{\mathrm{GC}}$ as

$$
\begin{aligned}
\dot{E}_{\mathrm{GC} \mathrm{SF}} & \gtrsim \mathrm{SFR}_{\mathrm{GC}} \times 1 \mathrm{SN} /\left(90 M_{\odot}\right) \times 10^{51} \mathrm{erg} / \mathrm{SN} \\
& =2.8 \times 10^{40} \mathrm{erg} \mathrm{s}^{-1}\left(\frac{\mathrm{SFR}_{\mathrm{GC}}}{0.08 M_{\odot} \mathrm{yr}^{-1}}\right),
\end{aligned}
$$

where a single core-collapse $\mathrm{SN}$ requires the formation of $\sim 90 M_{\odot}$ in stars (for a Kroupa 2001 initial mass function if the least massive star to explode has zero-age main-sequence mass of $8 M_{\odot}$ ), we assume $10^{51} \mathrm{erg}$ mechanical energy release per $\mathrm{SN}$, and we have normalized to a nuclear star formation rate of $\mathrm{SFR}_{\mathrm{GC}}=0.08 M_{\odot} \mathrm{yr}^{-1}$ (Crocker 2012).

Note that there are other significant star-formation-related sources of mechanical energy, including stellar winds and protostellar outflows, that could, in a full accounting, double this estimate. However, we adopt Equation (2.3) as our fiducial value for the injected power (see Lacki 2014). With this power, a conservative minimum timescale to inflate the bubbles (derived assuming that an unrealistically high $100 \%$ of mechanical energy injected in the nucleus is transferred to the bubbles and neglecting cooling and gravitational losses, which we show below are both actually important) is $t=H_{\mathrm{FB}} / \dot{E}_{\mathrm{GC} \mathrm{SF}} \simeq 8 \times 10^{7} \mathrm{yr}$. Given the current surface area of the bubbles, the expansion speed where $\dot{H}_{\mathrm{FB}}$ saturates the star formation mechanical power is $\sim 14 \mathrm{~km} \mathrm{~s}^{-1}$; this is an upper limit on the current expansion velocity of the bubbles' CD.

The total plasma mass in the bubbles of $M_{\mathrm{FB}} \sim$ $2.1 \times 10^{7} M_{\odot}$, if fed by the nuclear outflow at a mass inflow rate, $\dot{M}$, similar to the SFR, requires a minimum timescale to establish of $M_{\mathrm{FB}} / \dot{M}=2.6 \times 10^{8} \mathrm{yr} \times\left(0.08 M_{\odot} \mathrm{yr}^{-1}\right) / \dot{M}$. Normalizing to volumetric average values for plasma temperature and density obtained in Section 2.2, the cooling time of the plasma in the bubbles is $t_{\text {cool }}=3 / 2 k_{B} T /\left(\Lambda[T] n_{\text {tot }}\right) \simeq$ $5 \times 10^{8} \mathrm{yr} \times T_{\mathrm{FB}} /\left(3.5 \times 10^{6} \mathrm{~K}\right) \times\left(0.007 \mathrm{~cm}^{-3} / n_{\mathrm{tot}}^{\mathrm{FB}}\right)$, where ${ }^{9}$ $\Lambda[T]$ is the cooling function (Dopita et al. 2013; Sutherland et al. 2013) for plasma at temperature $T$. Thus, even the minimal inflation time is comparable to the radiative cooling time, indicating that an adiabatic treatment is inadequate. Note the confluence of timescales around the formation/maintenance of the bubbles at order $\sim$ few $\times 10^{8} \mathrm{yr}$ (see Crocker et al. 2014).

We show below (Section 4) that the bubbles approach a steady state such that their radii do not increase and that the freshly heated plasma mass flowing into them is matched by the dropout of cooling plasma, which condenses under a local thermal instability.

In such a steady state, material is advected from the cylindrical acceleration zone in the nucleus, of volume $V_{\mathrm{GC}}$

\footnotetext{
9 This timescale is a factor $\sim 2$ longer than that found by Crocker et al. (2014) the revised number is founded on the latest cooling curve calculation using the MAPPINGS IV v 4.2.5 NEQ cooling functions (R. Sutherland 2015, private communication; also see Dopita et al. 2013; Sutherland et al. 2013).
}

and radius $r_{0}$, by the wind, with speed $v_{\mathrm{w}}$, in a timescale $t_{\mathrm{w}}$, implying that a rate of mass loss of

$$
\dot{M}_{\mathrm{GC}} \equiv \mu \mathrm{m} n_{\mathrm{tot}}^{\mathrm{GC}} V_{\mathrm{GC}} / t_{\mathrm{w}}=\mu \mathrm{m} n_{\mathrm{tot}}^{\mathrm{GC}} 2 v_{\mathrm{w}} \pi r_{0}^{2}
$$

(where $m$ is the atomic mass unit and $\mu m$ is the mean mass per particle in the plasma) is identical to the rate of mass injection into the bubbles, which is, in turn, identical to the rate at which mass is cooling out of the structures,

$$
\dot{M}_{\text {cool }}=\mu \mathrm{m} n_{\text {tot }}^{\mathrm{FB}} V_{\mathrm{FB}} / t_{\text {cool }} \text {. }
$$

Hence, we estimate the radius of the nuclear region from which the outflow feeding the bubbles emerges as

$$
\begin{aligned}
r_{0} \simeq & \sqrt{\frac{V_{\mathrm{FB}} n_{\mathrm{tot}}^{\mathrm{FB}}}{2 \pi n_{\mathrm{tot}}^{\mathrm{GC}} v_{\text {wind }} t_{\mathrm{cool}}}} \simeq 70\left(\frac{v_{\text {wind }}}{500 \mathrm{~km} \mathrm{~s}^{-1}}\right)^{-1 / 2} \\
& \times\left(\frac{n_{\mathrm{tot}}^{\mathrm{FB}}}{0.004 \mathrm{~cm}^{-3}}\right)^{1 / 2}\left(\frac{n_{\mathrm{tot}}^{\mathrm{GC}}}{0.1 \mathrm{~cm}^{-3}}\right)^{-1 / 2} \mathrm{pc},
\end{aligned}
$$

where we normalize $n_{\text {tot }}^{\mathrm{GC}}$ to a value informed by GC X-ray observations (Muno et al. 2004). This is close to the radius of the central molecular zone (CMZ) molecular torus, $80-100 \mathrm{pc}$ (Molinari et al. 2011). The CMZ is the region of enhanced molecular gas density and star formation activity surrounding the central supermassive black hole, and the good agreement of our estimate of the outflow radius and the radius of this region supports our model that the bubbles are inflated by star formation over this region.

\section{LOCATION OF REVERSE SHOCKS}

In Section 4, we describe a numerical model for the expansion of a radiative bubble. An important feature of any bubble model is the location of the reverse shock, and in the case of the Fermi bubbles, the presence of such shocks has ramifications for the production of microwave and radio emission. We also show, in this section, that the reverse shock position affects our estimate of the energy flux into the bubbles, which is an important parameter in our later numerical modeling.

\subsection{Condition for Reverse Shock}

Let $\rho_{\mathrm{w}}$ and $v_{\mathrm{w}}$ be the density and velocity, respectively, of the Galactic center wind. The location of the reverse shock is determined from the condition that the ram pressure of the wind and the internal bubble pressure, $p_{\mathrm{b}}$, are related by the Rankine-Hugoniot condition for a strong shock ${ }^{10}$

$$
p_{\mathrm{b}}=\frac{2}{\gamma+1} \rho_{\mathrm{w}} v_{\mathrm{w}}^{2}
$$

where $\gamma=5 / 3$. On each side of the nucleus, we approximate the wind as conically expanding with solid angle $\Omega_{\mathrm{w}}$ so that the mass flux is

$$
\dot{M}=\Omega_{\mathrm{w}} R^{2} \rho_{\mathrm{w}} v_{\mathrm{w}},
$$

\footnotetext{
${ }^{10}$ Our modeling below demonstrates that the real shocks are, as assumed here, relatively strong, with the upstream flow having a Mach number in the range $6-8$.
} 
where $R$ is the spherical radius. Hence, the location of the shock is given by

$$
R_{\text {shk }}=\left(\frac{2}{\gamma+1} \frac{\dot{M} v_{\mathrm{w}}}{p_{\mathrm{b}} \Omega}\right)^{1 / 2} .
$$

Normally for a starburst wind, with initial power $\dot{E}$ and mass flux $\dot{M}$, we would assume that the wind velocity, $v_{\mathrm{w}} \simeq(2 \dot{E} / \dot{M})^{1 / 2}$, is constant. In this case the shock location would be given by

$$
R_{\text {shk }}=\frac{2^{3 / 4}}{(\gamma+1)^{1 / 2}} \frac{(\dot{E} \dot{M})^{1 / 4}}{\left(p_{\mathrm{b}} \Omega\right)^{1 / 2}} .
$$

However, the Galactic center wind, with a kinetic power $\sim 10^{40} \mathrm{ergs} \mathrm{s}^{-1}$, is relatively weak, compared to, say, the $\sim 10^{42} \mathrm{erg} \mathrm{s}^{-1}$ wind from the dwarf starburst galaxy M82 (Shopbell \& Bland-Hawthorn 1998; Cooper et al. 2008), and in the case of the Galactic center, gravity reduces the velocity of the wind on scales $\sim \mathrm{kpc}$.

\subsection{Effect of Gravitational Potential on the Wind Energy Flux and Velocity}

In order to estimate the effect of gravity on the energy flux and the wind velocity, we begin with the equation for the conservation of energy. In the following $\epsilon_{\mathrm{w}}$ is the internal energy density of the wind, $\phi$ is the gravitational potential, and $h_{\mathrm{w}}$ is the specific enthalpy. We have

$$
\begin{aligned}
& \frac{\partial}{\partial t} {\left[\frac{1}{2} \rho_{\mathrm{w}} v_{\mathrm{w}}^{2}+\epsilon_{\mathrm{w}}+\rho_{\mathrm{w}} \phi\right] } \\
&+\frac{\partial}{\partial x_{i}}\left[\left(\frac{1}{2} v_{\mathrm{w}}^{2}+h_{\mathrm{w}}+\phi\right) \rho_{\mathrm{w}} v_{\mathrm{w}, i}\right]=0 .
\end{aligned}
$$

Integrating over a volume bounded by streamlines and capped at the top by the reverse shock $(S)$ and below by a surface near the base of the flow $\left(S_{0}\right)$, we have, for a stationary flow, a conserved energy flux,

$$
\begin{aligned}
F_{E} & =\int_{S}\left(\frac{1}{2} v_{\mathrm{w}}^{2}+h_{\mathrm{w}}+\phi\right) \rho_{\mathrm{w}} v_{\mathrm{w}, i} n_{i} d S \\
& =\int_{S_{0}}\left(\frac{1}{2} v_{\mathrm{w}, 0}^{2}+h_{\mathrm{w}, 0}+\phi_{0}\right) \rho_{\mathrm{w}, 0} v_{\mathrm{w}, i, 0} n_{i, 0} d S_{0} .
\end{aligned}
$$

Denoting the hydrodynamic part of the energy flux by

$$
\dot{E}=\int_{S}\left(\frac{1}{2} v_{\mathrm{w}}^{2}+h_{\mathrm{w}}\right) \rho_{\mathrm{w}} v_{\mathrm{w}, i} n_{i} d S,
$$

we have that

$$
\dot{E}=\dot{E}_{0}-\int_{S} \rho_{\mathrm{w}} \phi v_{\mathrm{w}, i} n_{i} d S+\int_{S_{0}} \rho_{\mathrm{w}, 0} \phi_{0} v_{\mathrm{w}, 0, i} n_{i, 0} d S_{0} .
$$

Assuming that the bounding surfaces are approximately equipotential, and taking $\Delta \phi=\phi-\phi_{0}$ as the difference in potential between the two surfaces, we have

$$
\dot{E}=\dot{E}_{0}-\dot{M} \Delta \phi=\dot{E}_{0}\left(1-\frac{\dot{M}}{\dot{E}_{0}} \Delta \phi\right) .
$$

The factor $1-\dot{M} \Delta \phi / \dot{E}_{0}$ determines the reduction in hydrodynamic power resulting from the gravitational field.
We now use Bernoulli's equation to relate the velocity, specific enthalpy, and potential on $S$ with the corresponding

$$
\frac{1}{2} v_{\mathrm{w}}^{2}+h_{\mathrm{w}}+\phi=\frac{1}{2} v_{\mathrm{w}, 0}^{2}+h_{\mathrm{w}, 0}+\phi_{0}
$$

and approximate $\dot{E}_{0}$ by

$$
\dot{E}_{0} \simeq \dot{M} \times\left(\frac{1}{2} v_{\mathrm{w}, 0}^{2}+h_{\mathrm{w}, 0}\right) .
$$

This implies that

$$
v_{\mathrm{w}}^{2}+2 h_{\mathrm{w}} \simeq 2 \frac{\dot{E}_{0}}{\dot{M}}-2 \Delta \phi .
$$

As the flow expands, the enthalpy term diminishes in importance compared to the kinetic term (i.e., $h_{\mathrm{w}} \ll v_{\mathrm{w}}^{2} / 2$ ). Hence,

$$
v_{\mathrm{w}} \simeq\left(\frac{2 \dot{E}_{0}}{\dot{M}}\right)^{1 / 2}\left(1-\frac{\dot{M}}{\dot{E}_{0}} \Delta \phi\right)^{1 / 2} .
$$

Without the gravitational field, the asymptotic wind speed would be $\left(2 \dot{E}_{0} / \dot{M}\right)^{1 / 2} \simeq 630\left(\dot{E}_{0} / 5 \times 10^{39} \mathrm{ergs} \mathrm{s}^{-1}\right)^{1 / 2}$ $\left(\dot{M} / 0.04 M_{\odot} \mathrm{yr}^{-1}\right)^{-1 / 2} \mathrm{~km} \mathrm{~s}^{-1}$. The factor $\left(1-\dot{M} \Delta \phi / \dot{E}_{0}\right)^{1 / 2}$ determines the reduction in wind speed resulting from the gravitational potential.

\subsection{Location of the Reverse Shocks in the Presence of Gravity}

The factor $\left(1-\dot{M} \Delta \phi / \dot{E}_{0}\right)$, appearing in Equation (3.13), depends on the location of the reverse shock through the dependence of the potential difference $\Delta \phi$ on cylindrical coordinates, based on the Galactic center. We now describe how we determine the approximate location of the reverse shock and the hydrodynamic energy flux there.

Using Equation (3.1), relating the bubble pressure $\left(p_{\mathrm{b}}\right)$ and the wind ram pressure, and (3.2) for the mass flux, together with Equation (3.13) for the wind velocity, we have for the radial location of a spherical shock in a conically expanding wind:

$$
r_{\mathrm{shk}}=\left(\frac{2}{\gamma+1}\right)^{1 / 2} \frac{\left(2 \dot{E}_{0} \dot{M}\right)^{1 / 4}}{\left(p_{\mathrm{b}} \Omega\right)^{1 / 2}}\left(1-\frac{\dot{M}}{\dot{E}_{0}} \Delta \phi\right)^{1 / 4} .
$$

For the potential of the Galaxy, we use the analytic forms and parameters provided by Breitschwerdt et al. (1991). For the combined disk and bulge potential difference from the center of the Galaxy, expressed in $\left(\mathrm{km} \mathrm{s}^{-1}\right)^{2}$, as a function of cylindrical coordinates $r$ and $z$, both in $\mathrm{kpc}$, this gives

$$
\begin{aligned}
\Delta \phi[r, z] \simeq & 1.60 \times 10^{5}-\frac{8.82 \times 10^{4}}{\sqrt{r^{2}+z^{2}+0.245}} \\
& -\frac{1.10 \times 10^{6}}{\sqrt{r^{2}+\left(\sqrt{z^{2}+0.270}+7.26\right)^{2}}} \\
& +\frac{5.81 \times 10^{5}}{13+\sqrt{r^{2}+z^{2}}}+4.47 \\
& \times 10^{4} \ln \left[13+\sqrt{r^{2}+z^{2}}\right]\left(\mathrm{km} \mathrm{s}^{-1}\right)^{2} .
\end{aligned}
$$




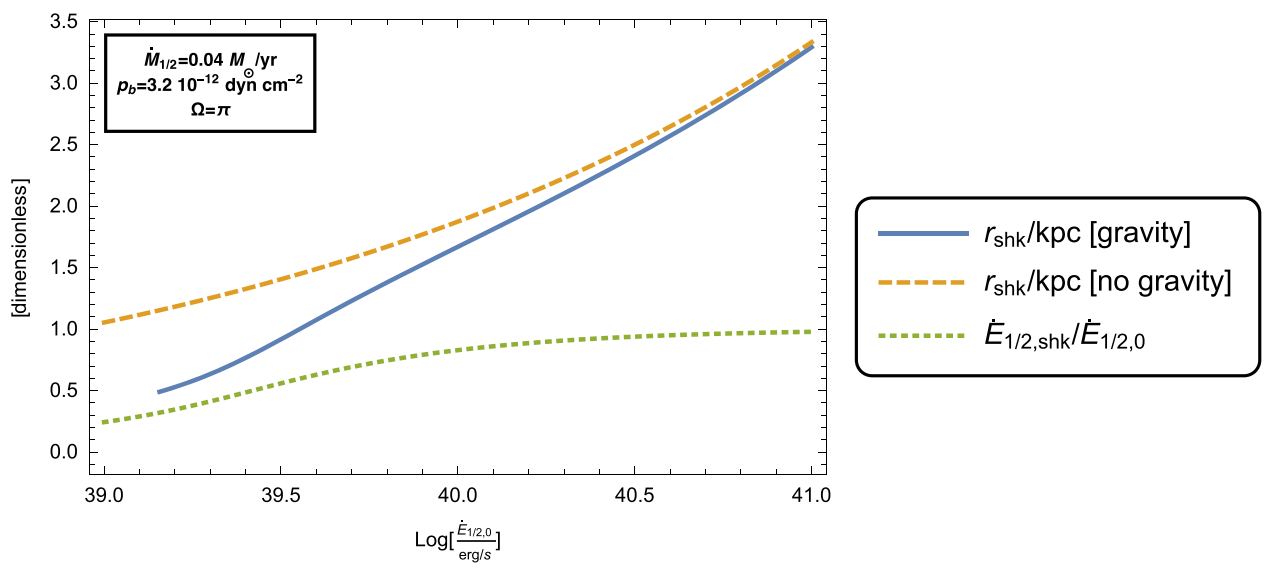

Figure 2. Distance to the shock and relative mechanical power at the shock as a function of mechanical power injected at the nucleus into one hemisphere, $\dot{E}_{1 / 2,0}$. The downstream thermal pressure in the bubble is $2 \mathrm{eV} \mathrm{cm}^{-3} \simeq 3.2 \times 10^{-12} \mathrm{dyn} \mathrm{cm}^{-2}$, the mass flux into the flow is $0.04 M_{\odot} \mathrm{yr}^{-1}$, and we adopt $\Omega \simeq \pi($ Lacki 2014 ), corresponding to an expansion cone angle of $\sim 120^{\circ}$.

Notwithstanding the expression for $\Delta \phi$ being in terms of cylindrical coordinates, the contours of $\Delta \phi$ are approximately spherical in the region of interest $\left(r_{\mathrm{shk}} \lesssim 1.5 \mathrm{kpc}\right)$. Hence, in solving Equation (3.14) we have approximated $\Delta \phi$ by its value on the $z$-axis, i.e., $\Delta \phi\left[0, r_{\text {shk }}\right]$.

In Figure 2 we show, as a function of the mechanical power injected at the nucleus into one hemisphere (for the parameters specified in the caption), our numerical solution for (i) the distance to the shock in $\mathrm{kpc}$ (with and without gravitational deceleration of the flow) and (ii) the relative mechanical power available at the shock (given gravitational deceleration). It is evident that for the chosen parameter values, which are apposite to the nuclear outflow that feeds the Fermi bubbles, the flow can lose $30 \%-50 \%$ of its mechanical power to gravity before reaching the shock.

\section{EXPANSION OF A RADIATIVE BUBBLE}

We now describe our calculation of the expansion of a bubble into an atmosphere of finite pressure incorporating the dynamical effect of energy losses due to cooling radiation emitted by the shocked material located between reverse shock and $\mathrm{CD}$. We demonstrate here that, for parameters relevant to the Fermi bubbles, we expect the $\mathrm{CD}$ to be very slowly expanding (in line with the X-ray data) at only $\sim$ few $\mathrm{km} \mathrm{s}^{-1}$ and for the structures to have reached a size very similar to that observed.

\subsection{Spherical Bubble Model}

As a first approximation to modeling the Fermi bubbles, we treat them as two hemispheres of a single spherical bubble driven by a spherically symmetric wind propagating into a constant-density, constant-pressure medium. In the observed Fermi bubbles the morphology close to the Galactic plane is probably constrained by the larger density and pressure there. In comparing the radius of this model with the extent of the Fermi bubbles, we calculate an equivalent spherical radius $r_{\mathrm{FB}}=\left[3 /(4 \pi) V_{\mathrm{FB}}\right]^{1 / 3} \simeq 4.1 \mathrm{kpc}$, where $V_{\mathrm{FB}}=285 \mathrm{kpc}^{3}$ is the estimated volume. Our model generalizes the usual treatment of a wind-driven bubble (e.g., Weaver et al. 1977), in that the bubble is not assumed to be strongly overpressured with respect to the ambient medium and is also subject to significant

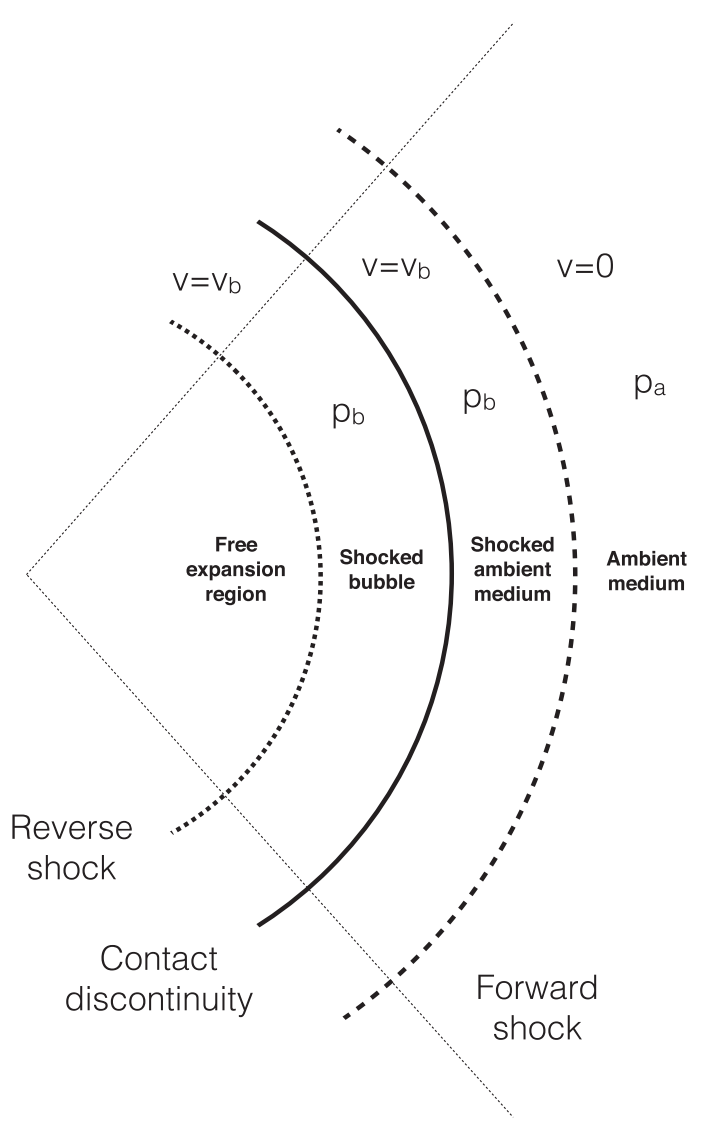

Figure 3. Illustration of the key features of a bubble.

optically thin radiative cooling. The model in this section does not incorporate a gravitational potential (so that there is no internal or external pressure stratification) and, in common with other bubble models, neglects the small but finite fraction of the total bubble volume in the region upstream of the internal reverse shock.

Figure 3 lays out the main features of such a bubble: there is a reverse shock interior to the bubble, where the decreasing ram pressure of the spherically expanding, supersonic nuclear wind 


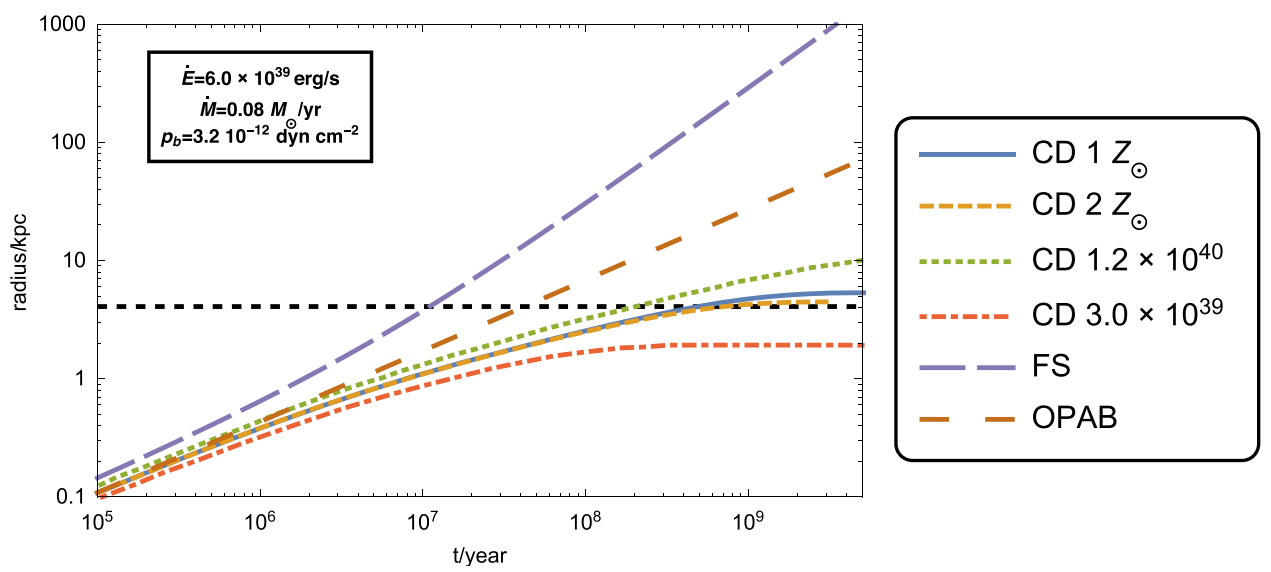

Figure 4. Radius of the bubble contact discontinuity (CD) and forward shock (FS) vs. time for $\dot{E}=6 \times 10^{39} \mathrm{erg} \mathrm{s}^{-1}$ and solar metallicity (unless otherwise marked) and $\dot{M}=0.08 M_{\odot} \mathrm{yr}^{-1}$ (fixed); the atmosphere has pressure $2.0 \mathrm{eV} \mathrm{cm}^{-3} \simeq 3.2 \times 10^{-12} \mathrm{dyn} \mathrm{cm}^{-2}$. CD curves are (i) solid blue for $\dot{E}=6.0 \times 10^{39} \mathrm{erg} \mathrm{s}^{-1}$; (ii) dashed orange for $\dot{E}=6.0 \times 10^{39} \mathrm{erg} \mathrm{s}^{-1}$ and cooling with twice solar metallicity $\left(Z=2 Z_{\odot}\right)$; (iii) dotted green for $\dot{E}=1.2 \times 10^{40} \mathrm{erg} \mathrm{s}^{-1}$; and (iv) dot-dashed orange for $\dot{E}=3.0 \times 10^{39} \mathrm{erg} \mathrm{s}^{-1}$. The long-dashed violet curve is for the forward shock. The brown long-dashed curve shows the analytical result for the expansion of an overpressured, adiabatic bubble (OPAB; see the Appendix); this very substantially overestimates the bubble radius for $t \gtrsim$ few $\times 10^{7}$ yr. The horizontal black dashed line represents the equivalent spherical radius of $4.1 \mathrm{kpc}$ corresponding to the total volume of the Fermi bubbles; for the fiducial model the bubble has attained this size by $\sim 5 \times 10^{8} \mathrm{yr}$.

is balanced by the internal pressure of the bubble as described in Section 3, and a region of shock-heated bubble material downstream of the reverse shock, in which the internal energy dominates the kinetic energy. This region is bounded by a CD, external to which is a shell of shocked, ambient ISM between the forward shock, which is expanding into the external atmosphere, and CD. We approximate this region as one of constant spatial pressure and velocity and neglect cooling there. We also in this section approximate the region between $C D$ and reverse shock as constant in density. The bubble is fed by mechanical energy injected at a rate $\dot{E}$ and mass at a rate $\dot{M}$. We assume a constant $\gamma$-law equation of state, with the pressure, $p$, and internal energy density, $\epsilon$, related by $p=(\gamma-1) \epsilon$ and $\gamma=5 / 3$. It is important to note that the growth of the total mass of the bubble $M_{\mathrm{b}}$ does not satisfy $\dot{M}_{\mathrm{b}}=\dot{M}$ because of the phenomenon of mass dropout driven by cooling (Crocker et al. 2014). We estimate the rate of mass dropout by equating the post-reverse-shock enthalpy flux of that mass with the cooling inside the bubble.

The equations governing the pressure, radius, mass, and density of the bubble are derived in the Appendix, using energy, mass, and momentum conservation integrated over the various regions identified above. We initiate the evolution of the bubble at $t=10^{5} \mathrm{yr}$ with initial values defined by an adiabatic bubble (see the Appendix for details).

Cooling sets up local thermal instability of the bubble plasma, leading it to collapse into overdense condensations that, having lost buoyancy (in a real situation that includes a gravitational potential), fall out of the bubble (see Crocker et al. 2014 and references therein).

As the bubble's pressure decreases as a result of the combined effect of the work done by expansion and the radiant loss of energy, it can reach a steady state where its pressure equilibrates with the surrounding atmosphere, the energy input is balanced by thermal radiation, and the mass flux is balanced by mass dropout. We show below that, for parameters appropriate to the Fermi bubbles, the system is approaching this limit.

\subsection{Evolution of a Spherical Bubble with Parameters Relevant to the Galactic Center}

On the basis of the numbers presented in Section 2.3, we choose as representative values for the total mass and mechanical power injected at the nucleus $\left(\dot{E}_{0}, \dot{M}\right) \sim$ $\left(10^{40} \mathrm{erg} \mathrm{s}^{-1}, 0.08 M_{\odot} \mathrm{yr}^{-1}\right)$ (so that $\dot{E}_{1 / 2,0} \equiv 1 / 2 \times \dot{E}_{0}$ and $\dot{M}_{1 / 2} \equiv 1 / 2 \times \dot{M}$ is injected into the flow into each hemisphere); the exact parameter range allowable for these quantities is delimited in Section 5. One must remember, however, that in Section 3.3 we showed that, for parameter values in this range, the flow into each bubble can lose $~ 30 \%-$ $50 \%$ of its mechanical power to gravity before reaching the shocks. For purposes of comparing our current modelingwhich neglects gravity-with our previous (and subsequent) results, we now therefore choose an injected mechanical power that is reduced by $40 \%$ with respect to the fiducial value nominated above, namely, $6 \times 10^{39} \mathrm{erg} \mathrm{s}^{-1}$. Our purpose here is to show that, for parameters relevant to the Fermi bubbles, the expansion of a radiative bubble (into an atmosphere of pressure similar to that of the Galactic halo) quickly slows down (consistent with the lack of X-ray brightening at the edges of the Fermi bubbles) and, moreover, there is an asymptotic radius for such a bubble that is not much larger than the equivalent radius of the observed Fermi bubbles.

As justified above, another relevant parameter is an ambient pressure of $2.0 \mathrm{eV} \mathrm{cm}^{-3}\left(=3.2 \times 10^{-12} \mathrm{dyn} \mathrm{cm}^{-2}\right)$. Note that we assume that the plasma has solar metallicity but verify that, given that the metallicity affects the cooling, assuming twice solar metallicity does not change our results significantly (see Figure 4).

Figure 4 shows the evolution of the bubble radius (i.e., $\mathrm{CD}$ ) for input parameters $\dot{E}=6.0 \times 10^{39} \mathrm{erg} \mathrm{s}^{-1}$ and $\dot{M}=0.08 M_{\odot}$. $\mathrm{yr}^{-1}$ (i.e., $1.2 \times 10^{15} \mathrm{erg} \mathrm{g}^{-1}$ as the energy per mass content of the outflow), as well as the locus of the forward shock. The radius of a highly overpressured bubble and the equivalent spherical radius of the Fermi bubbles are also shown for comparison. Clearly, for the fiducial parameters the bubble radius asymptotes to approximately $5 \mathrm{kpc}$ after about 


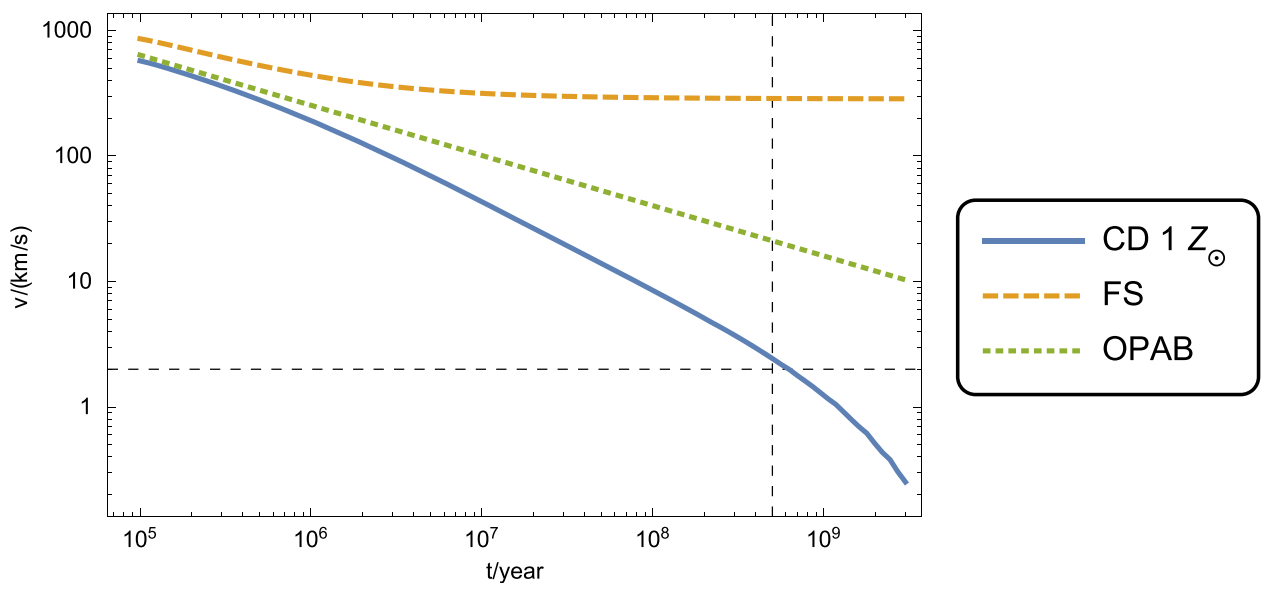

Figure 5. Velocity of the bubble contact discontinuity $(\mathrm{CD})$ and forward shock (FS) vs. time for cooling with $Z=Z_{\odot}$. Parameters as for Figure 4 . The dotted (green) curve shows the analytical result for the expansion of the $\mathrm{CD}$ of an overpressured, adiabatic bubble (OPAB). The expansion velocity of the forward shock asymptotes to the sound speed as it degenerates into a sound wave. For the chosen parameters, at the age $\left(\sim 5 \times 10^{8} \mathrm{yr}\right.$ indicated by the vertical line $)$ where the bubble has attained a volume equivalent to the observed Fermi bubbles (see Figure 4), its CD is expanding at $2-3 \mathrm{~km} \mathrm{~s}^{-1}$.

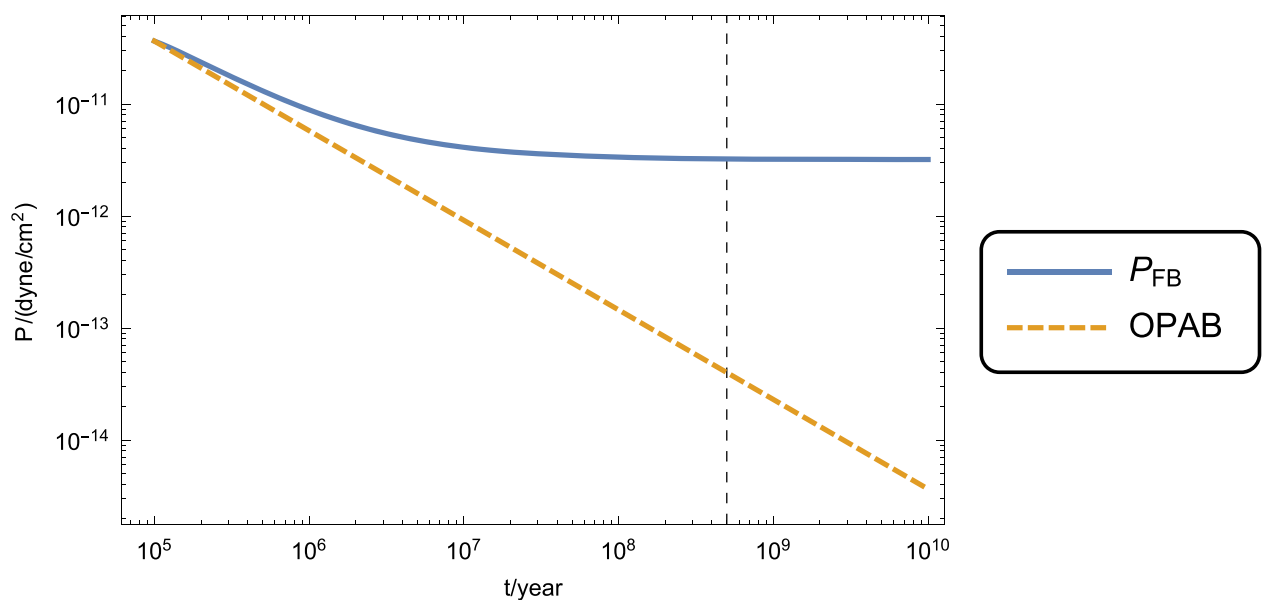

Figure 6. Pressure interior to the $\mathrm{CD}$ (solid blue); this asymptotes to the assumed atmospheric pressure $3.2 \times 10^{-12}$ dyn $\mathrm{cm}^{-2}$. Parameters as for Figure 4 . The vertical line indicates when the bubble has attained a volume equivalent to the observed Fermi bubbles (see Figure 4). The dashed orange curve is for an overpressured, adiabatic bubble.

$1 \mathrm{Gyr}$, and from about $3 \times 10^{8} \mathrm{yr}$ onward, the bubble radius is not far from the spherical equivalent radius $\simeq 4.1 \mathrm{kpc}$ of the observed bubbles. For comparison, for fixed $\dot{M}=0.08 M_{\odot}$ $\mathrm{yr}^{-1}$, cases of $\dot{E}=1.2 \times 10^{40} \mathrm{erg} \mathrm{s}^{-1}\left(2.4 \times 10^{15} \mathrm{erg} \mathrm{g}^{-1}\right)$ and $\dot{E}=3.0 \times 10^{39} \mathrm{erg} \mathrm{s}^{-1}\left(6.0 \times 10^{14} \mathrm{erg} \mathrm{g}^{-1}\right)$ are also shown in Figure 4. The former does not asymptote to a final radius of $\sim 10 \mathrm{kpc}$ until $10 \mathrm{Gyr}$ (with volume $\sim 15 \times V_{\mathrm{FB}}$ ), and it spends only a brief time in the vicinity of the currently observed size, tending to suggest a return to the "Why now?" problem encountered by explosive scenarios. The latter never reaches the size (or minimum temperature) of the observed Fermi bubbles.

The expansion of the bubble decelerates from approximately $2 \times 10^{6} \mathrm{yr}$ on, and this is clearly shown in Figure 5. From about $10^{8} \mathrm{yr}$ on, the bubble expansion velocity decelerates to less than $10 \mathrm{~km} \mathrm{~s}^{-1}$ as it approaches pressure equilibrium with the environment. The approach to pressure equilibrium is shown in Figure 6. At the same time, the forward shock becomes a sound wave propagating into the background medium at a sound speed of $\simeq 280 \mathrm{~km} \mathrm{~s}^{-1}$ (again see Figure 5).
When the bubble's growth saturates, its total mass and energy content are fixed, with the post-reverse-shock enthalpy flux balanced by the radiative luminosity. Figure 7 shows that the mass dropout saturates at the injection rate of $0.08 M_{\odot} \mathrm{yr}^{-1}$ and that, consistent with this, the net mass injection into the bubbles saturates at zero, maintaining the total mass interior to the bubbles at a constant value.

For comparison with other sections, we can estimate the final, asymptotic volume, $V_{\mathrm{f}}$, in this circumstance, with the input hydrodynamic energy flux, $\dot{E} \equiv 5 / 2 \dot{M} /(\mu \mathrm{m}) k_{B} T$, arriving at and processed through the shocks, balanced by radiative cooling and with the pressure in the downstream bubble region in equilibrium with the halo atmosphere. Here the post-reverseshock bubble temperature is $T=3 / 16 \mu \mathrm{m} / k_{B} v_{\mathrm{w}}^{2} \simeq$ $3.5 \times 10^{6}\left(v_{\mathrm{w}} / 500 \mathrm{~km} \mathrm{~s}^{-1}\right)^{2} \mathrm{~K}$; the total post-shock number density in the bubble is $n_{\text {tot }}=4 \dot{M} /\left(\mu \mathrm{m} \Omega R_{\mathrm{sh}}^{2} v_{\mathrm{w}}\right)$ (see Section 3 for the definitions of these symbols). Balancing the energy injected into the bubble with thermal cooling and normalizing to fiducial values for temperature and number density (such that pressure equilibrium with the halo is satisfied), we then 


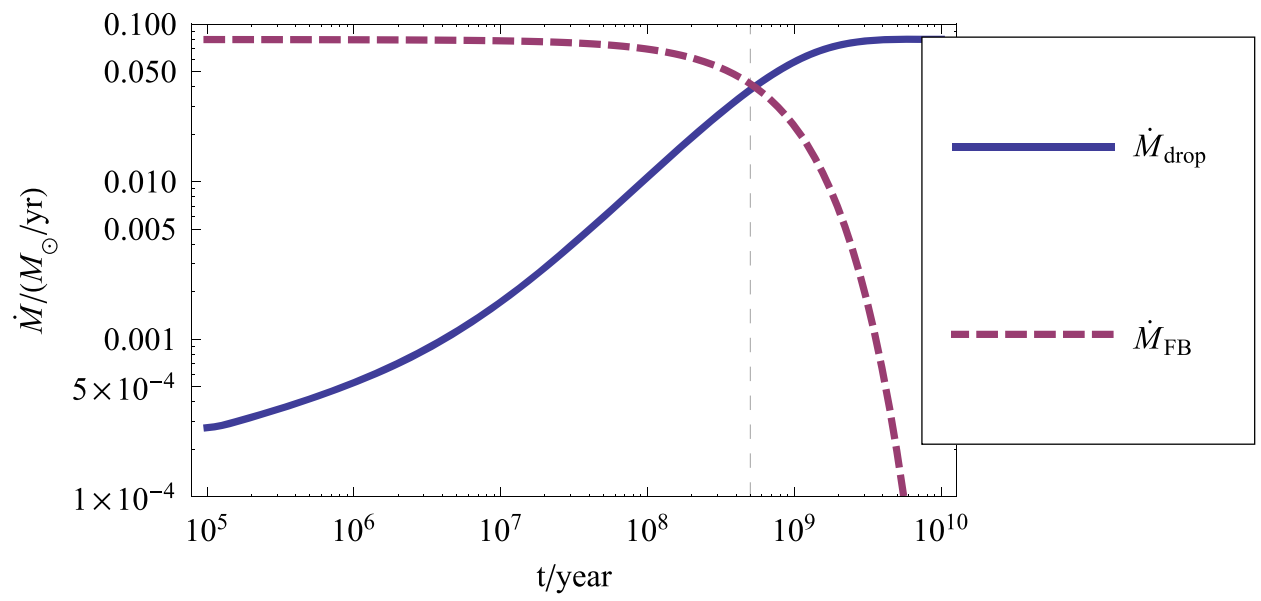

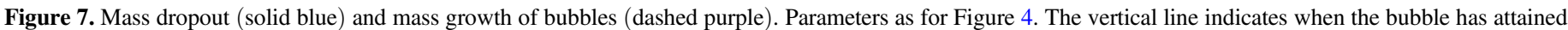
a volume equivalent to the observed Fermi bubbles (see Figure 4). At times $\gtrsim 1$ Gyr mass dropout by itself saturates the injection rate of freshly heated plasma.

have that the final volume of the bubble is

$$
\begin{aligned}
V_{\mathrm{f}} \simeq & \frac{\dot{E}}{n_{\mathrm{tot}}^{2} \Lambda[T]}=\frac{5 / 2 \dot{M} /(\mu \mathrm{m}) k_{B} T_{\mathrm{FB}}}{n_{\mathrm{tot}}^{2} \Lambda[T]} \\
= & 2.0 \times 10^{67} \mathrm{~cm}^{3}\left(\frac{T_{\mathrm{FB}}}{3.5 \times 10^{6} \mathrm{~K}}\right)\left(\frac{\dot{M}}{0.08 M_{\odot} \mathrm{yr}^{-1}}\right) \\
& \times\left(\frac{n_{\mathrm{tot}}}{0.0066 \mathrm{~cm}^{-3}}\right)^{-2}\left(\frac{\Lambda[T]}{10^{-23.15} \mathrm{erg} \mathrm{cm}^{3} \mathrm{~s}^{-1}}\right)^{-1}
\end{aligned}
$$

where we normalize to an $\dot{M}$ favored by the modeling presented in Section 5. This is $\sim 2.3 \times$ the observationally estimated volume of the Fermi bubbles, $V_{\mathrm{FB}}=8.2 \times 10^{66} \mathrm{~cm}^{-3}$, consistent with our expectation that the bubbles are still slowly expanding. Note that the estimated volume would be smaller if the increase in density of cooling material in the shell region (R3) were taken into account.

\section{SCAN OVER PARAMETER SPACE}

We have shown in the previous section that (i) the bubbles are expected to contain giant, reverse shocks at heights of $\sim 1 \mathrm{kpc}$ above the nucleus and (ii) the bubbles are likely to be slowly expanding structures close to pressure equilibrium with the surrounding halo. We now explore the implications of this general picture more quantitatively. In particular, in this section we determine the allowable ranges for the rate at which mechanical energy and mass are fed into the bubbles and thermalized at the reverse shocks; in the subsequent section we examine acceleration of cosmic rays at these shocks and their attendant, nonthermal radiation.

\subsection{Procedure}

We now explain our procedure for scanning over the steady mass and mechanical energy flux, $\dot{M}$ and $\dot{E}_{0}$, respectively, into the central acceleration region. As shown above, these parameters determine the temperature reached within the central acceleration region following thermalization of the mechanical energy. The high pressure in this region accelerates the plasma into a flow that is collimated into a biconical wind transverse to the Galactic disk by the surrounding dense molecular torus of the CMZ. In this expanding flow, the plasma cools adiabatically until it reaches the reverse shock in each bubble, where it is shock (re)heated. For every $\left\{\dot{E}_{0}, \dot{M}\right\}$ in the parameter space, the asymptotic velocity of the wind, $v_{\text {asym }}$, is determined. Note that $v_{\text {asym }}$ is reached soon after the flow escapes from the central acceleration zone; subsequent to reaching $v_{\text {asym }}$, the adiabatically cooling flow decelerates in response to the gravitational potential, $\phi$ (Breitschwerdt et al. 1991), as described above. Given the high (i.e., supersonic) upstream flow speeds in which we are interested, we treat the flow evolution in the ballistic limit, where we ignore small corrections due to the temperature evolution of the upstream gas.

In our modeling, for every sampled $\left\{\dot{E}_{0}, \dot{M}\right\}$ point, we determine (i) the height of the reverse shocks $R_{\text {sh }}$ and (ii) the temperature of the shocked material, $T_{\mathrm{sh}}$. Given that, observationally, the bubbles do not expand latitudinally very much at heights greater than the expected position of each shock, we neglect further possible adiabatic plasma temperature losses with height above the shocks. We also assume a uniform plasma temperature in $\mathrm{R} 2$ given by that for a strong shock with upstream flow velocity $v_{\mathrm{w}}$ and $\gamma=5 / 3$. Hence, $T_{\mathrm{FB}}=T_{\mathrm{sh}}=3 / 16 \mu \mathrm{m} v_{\mathrm{w}}^{2} / k_{B}$.

The shocks deliver a fraction, $\epsilon_{\mathrm{CR}}$, of their total mechanical power (accounting for gravitational losses), $\dot{E}$, into freshly accelerated cosmic rays: $L_{\mathrm{CR}} \equiv \epsilon_{\mathrm{CR}} \times \dot{E}$; the spectral index of the cosmic rays' power-law distribution is controlled by the Mach number of the shocks. We shall see subsequently that, a posteriori, we are warranted in assuming strong shocks: the hard, nonthermal spectra of both the $\gamma$-ray emission from the Fermi bubbles and the microwave haze require shock Mach numbers of $M \gtrsim 4$ (see Section 6). We also find (see Section 6.2.2) that given that nonthermal ISM phases dominate the dynamics of the compression shell R3, its size is dependent on the efficiency with which the shocks accelerate cosmic rays, $\epsilon_{\mathrm{CR}}$ (see Equation (6.9)). We have explored a range of values for this parameter. Also important are the pressure of the atmosphere into which the bubbles are expanding and the effective temperature of the compression shell. For the former our fiducial value is $P_{\text {halo }}=2 \mathrm{eV} \mathrm{cm}^{-3}=3.2 \times 10^{-12} \mathrm{erg} \mathrm{cm}^{-2}$ adopted from Section 2.2, and, for the latter, following Section 2.2, we fix $T_{\text {shell }}=3.5 \times 10^{6} \mathrm{~K}$ (with $T_{\mathrm{FB}}$ somewhat larger in general). 


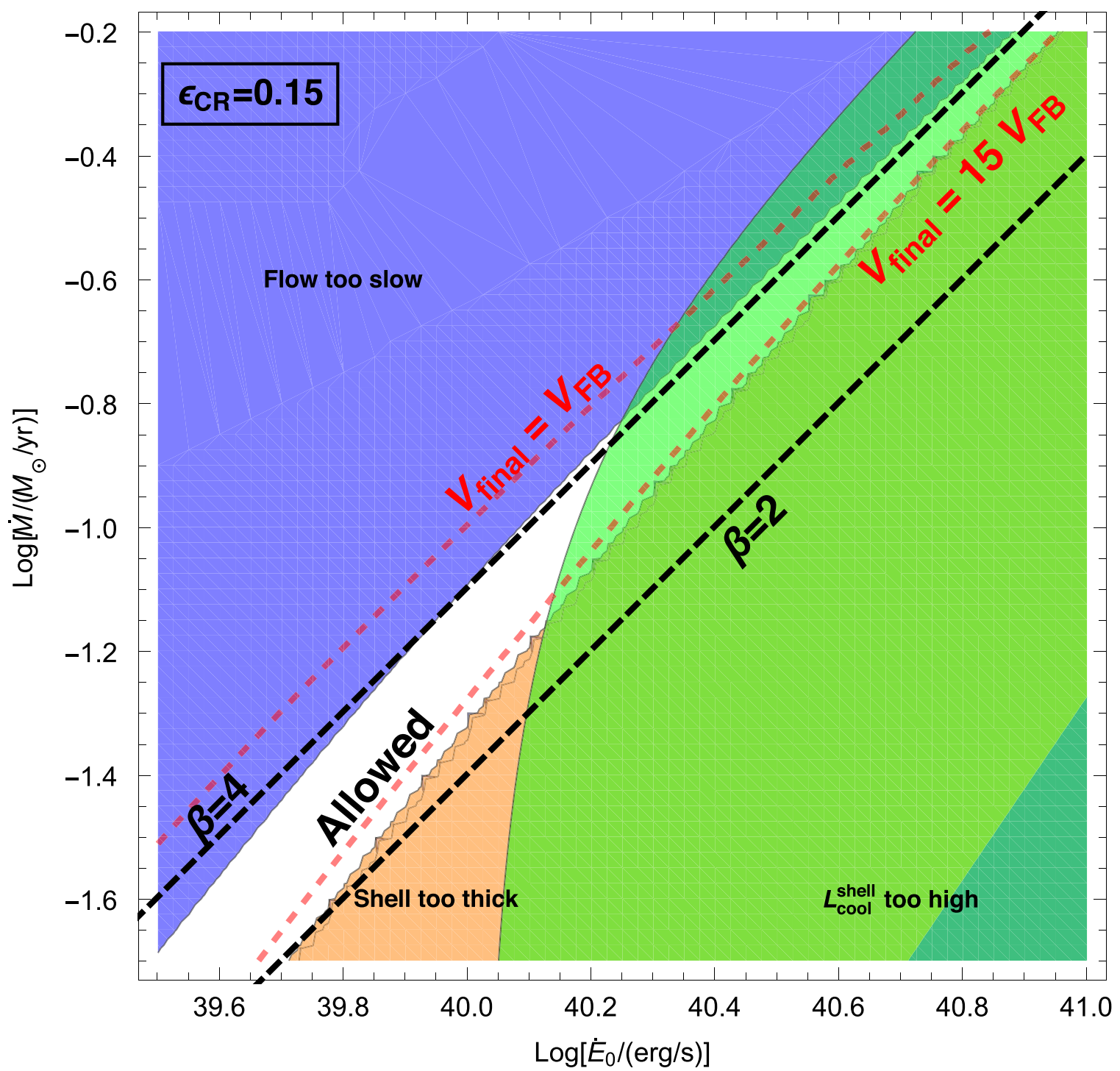

Figure 8. Explored $\left\{\dot{E}_{0}, \dot{M}\right\}$ parameter space and excluded regions for the case that $P_{\text {halo }}^{F B}=2 \mathrm{eV} \mathrm{cm}^{-3}, \epsilon_{\mathrm{CR}}=0.15$, and $T_{\text {shell }}=3.5 \times 10^{6} \mathrm{~K}$. White space shows the allowed region. The region masked out in green predicts a bolometric cooling luminosity $\left(>10^{40} \mathrm{erg} \mathrm{s}^{-1}\right)$ from the compression shell (R3) that is too high to be compatible with X-ray constraints from ROSAT (Snowden et al. 1997; Almy et al. 2000). The region masked out in orange predicts a compression shell that is too thick $(>1.4 \mathrm{kpc})$. The region masked out in blue predicts that the flow from the interior region into the shell is too slow, i.e., the transport time from R2 to R3 is longer than the plasma cooling time in R2, so the plasma cools before it reaches the shell, at variance with the assumptions of the model. The thick black dashed curves delineate the star-formation-driven mass efflux vs. mechanical energy input into the giant outflows for mass loading rates of $\beta=2$ and 4 , scaling the results of Strickland \& Heckman (2009). The dashed red lines trace the locus of $\dot{E}_{0} / \dot{M}$ points that generate asymptotic volumes of, respectively, $V_{\mathrm{FB}}$ and $15 \times V_{\mathrm{FB}}$ obtained from Equation (4.1).

\subsection{Restriction of Parameter Space}

With the downstream temperature and shock height corresponding to every $\left\{\dot{E}_{0}, \dot{M}\right\}$ point determined, we can determine a number of subsequent quantities, including the Mach number of the shock, the bolometric thermal luminosity of the bubbles, and the thickness of cooling material in the compression shell leading up to the $\mathrm{CD}$ from the inside (region $\mathrm{R} 3$ in Figure 1). By confronting these modeled values with physical or observational constraints, we can exclude much of the $\left\{\dot{E}_{0}, \dot{M}\right\}$ parameter space; see Figure 8 . In this figure we show the case that the downstream, shocked plasma in region R2 has reached pressure equilibrium with an exterior atmosphere of $P_{\text {halo }}=2 \mathrm{eV} \mathrm{cm}^{-3}$, the fraction of mechanical power available at the shocks going into cosmic rays is $\epsilon_{\mathrm{CR}}=0.15$, and the compression shell at R3 has an effective temperature of
$T_{\text {shell }}=3.5 \times 10^{6} \mathrm{~K}$. Most of the potential parameter space for these parameters can be excluded by only three considerations.

1. The compression shell should not be too thick; the requirement of a flat, projected surface brightness in $\gamma$ rays leads to a geometric restriction that the shell thickness, $d_{\text {shell }}$, satisfies $d_{\text {shell }}<0.456 r_{B} \sim 1.4 \mathrm{kpc}$ (see Section 6.2.5). This excludes the green region in Figure 8. Note that the shell thickness is determined by the generalization of Equation (6.10) below.

2. The time for the gas to flow from the shocked interior (R2) into the compression shell (R3) (at speed given by Equation (6.4) below) should be shorter than the radiative cooling time in R2 (otherwise, cooling takes place in R2, not in R3, at variance with the assumptions of the model). 
3. The total cooling luminosity, $L_{\text {cool }}^{\text {shell }}$ of the compression shell, R3, should not exceed the upper limit on the bolometric luminosity of the X-ray bulge, which, conservatively, is $10^{40} \mathrm{erg} \mathrm{s}^{-1}$ (Snowden et al. 1997; Almy et al. 2000).

Note that while, in principle, the allowed parameter space seems to extend off the plot toward the lower left, there are two considerations that exclude $\dot{E}_{0} \lesssim 3 \times 10^{39} \mathrm{erg} \mathrm{s}^{-1}$, at least in the context of the current work: (i) within a hadronic model, the minimum (thick target) cosmic-ray luminosity necessary to sustain the bubbles' $\gamma$-ray luminosity is $\sim 3 \times 10^{38} \mathrm{erg} \mathrm{s}^{-1}$ (Crocker \& Aharonian 2011); a mechanical power at least a factor of $\sim 3$ (for high cosmic-ray acceleration efficiency) larger than this has to be be delivered at the shocks in order to sustain their emission, and one must then factor in gravitational losses (see Figure 2); (ii) we show below (see Figure 12) that, in order to reproduce the giant outflows' synchrotron luminosity, an unreasonably large fraction of the total cosmic-ray luminosity of the shocks has to go into cosmic-ray electrons for $\dot{E}_{0} \lesssim 3 \times 10^{39} \mathrm{erg} \mathrm{s}^{-1}$.

We show below that, within the restricted parameter space we have mapped out, our model well reproduces the $\gamma$-ray, microwave, and radio phenomenology of the bubbles. First, however, we remark on three interesting findings.

First, the $\dot{M} / \dot{E}_{0}$ ratio indicated by this restricted parameter space is again entirely consistent with-and, indeed, indicative of - the bubbles being driven by nuclear star formation. In Figure 8 the overplotted black curves delineate expected starformation-driven mass flux versus star-formation-driven mechanical energy input for mass loading rates, as marked, of $\beta=2$ and 4 that bracket our allowable parameter space (scaling results of Strickland \& Heckman 2009). Here mass loading is defined with respect to the mass injection rate from $\mathrm{SN}$ and stellar winds, $\dot{M}_{\mathrm{SN}+\mathrm{Sw}}$, that accompanies a given rate of star formation, SFR, not with respect to this star formation rate itself: $\dot{M}_{\mathrm{SN}+\mathrm{SW}}=\dot{M}_{\mathrm{SN}+\mathrm{SW}}[\mathrm{SFR}]$ with $\dot{M}_{\mathrm{tot}} \equiv \beta \times \dot{M}_{\mathrm{SN}+\mathrm{SW}}$ (Strickland \& Heckman 2009). From modeling of the diffuse, hard X-ray emission emanating from the starburst exemplar M82, Strickland \& Heckman (2009) find-for centralized mass loading only-mass loading rates in the range $1<\beta<2.8$ with a practical upper limit at $\beta \sim 10$ for a very hot, $(30-70) \times 10^{6} \mathrm{~K}$, wind. The near coincidence of our allowable parameter space with a scale for the $\beta$ parameter derived from modeling other star-formation-driven outflows (Strickland \& Heckman 2009, and references therein) supports the notion that the outflow is star formation driven; a priori there is no reason for an AGN-driven outflow to be anywhere close to this $\beta$ range.

Note that there is, in general, the possibility for an outflow to also be subjected to distributed (in addition to centralized) mass loading. The population of $\mathrm{H}$ I clouds entrained into the nuclear outflow detected by McClure-Griffiths et al. (2013) and the high-speed, warm ionized material found by Fox et al. (2014) together constitute plausible evidence that exactly such distributed mass loading is taking place in the nuclear outflow, presumably as it scrapes along the inside of the $\mathrm{CMZ}$ molecular gas torus. The fact that our allowable parameter space extends into regions corresponding to very slightly higher values of $\beta$ than determined by Strickland \& Heckman (2009) for M82 may reflect the occurrence of both centralized and distributed mass loading in generating the final effective $\beta$. Note that the pressure in the $\mathrm{CMZ} \mathrm{H}_{2}$ is very close to that in the central
X-ray-emitting plasma; the molecular gas is thus able to collimate this plasma and cause it to escape down the steepest density gradient, into the Galactic halo. ${ }^{11}$

A second interesting finding comes from consideration of the dashed red curves in Figure 8 . These show where the final volumes (obtained from the conditions of pressure equilibrium and balance between cooling radiation and incoming enthalpy flux according to Equation (4.1)) of spherical, radiative bubbles fed with energy and mass at the nominated rates are, respectively, equal to the current estimated volume of the observed Fermi bubbles and $15 \times$ the current volume. Left and upward of the $V_{\mathrm{FB}}$ curve, radiative bubbles never reach the current size of the observed Fermi bubbles, therefore also ruling out this region of parameter space. With increasing distance right and downward of the $15 V_{\mathrm{FB}}$ curve, there is an increasingly severe temporal fine-tuning problem as radiative bubbles spend only a brief time over their entire evolution close to the current observed size. We emphasize, then, that for the region of $\left\{\dot{E}_{0}, \dot{M}\right\}$ parameter space delimited by the independent considerations set out above, the Fermi bubbles are naturally found to have a size close to that observed. In other words, there is no "Why now?" problem in this model.

A final interesting finding is that allowable values of $P_{\text {halo }}$ and $\epsilon_{\mathrm{CR}}=0.15$ are quite tightly constrained within our model: we are not able to reproduce the observed $\gamma$-ray luminosity of the bubbles (see Section 6 below) for $P_{\text {halo }} \lesssim 1.5 \mathrm{eV} \mathrm{cm}^{-3}$; for $\epsilon_{\mathrm{CR}} \gtrsim 0.3$ we find that the parameter space allowed by the three considerations listed above closes off completely, and for $\epsilon_{\mathrm{CR}}$ $\lesssim 0.05$ we cannot simultaneously reproduce both the observed $\gamma$-ray and radio+microwave luminosities of the giant outflows (see below). This allowable range for $\epsilon_{\mathrm{CR}}$ is consistent with independent determinations of the fraction of shock mechanical power that goes into such particles in other systems (e.g., Zirakashvili \& Völk 2006, on the nuclear outflow from local starburst NGC 253).

We lastly note that, in the allowed parameter space, cooling in the shell does not saturate the mechanical energy injected in the nucleus; this means that the $\mathrm{CD}$ must be expanding and thereby doing work on its surroundings at a rate $P \dot{V}$ that saturates the residual mechanical energy. The bubbles, therefore, have not yet reached their asymptotic, final volume. In fact, we find that the CD must be expanding at about $2 \mathrm{~km} \mathrm{~s}^{-1}$ to saturate the injected power not lost to cooling radiation in the shell. Comparing against the results from Section 4, the spherical radiative bubble is expanding this quickly at an age of $5 \times 10^{8} \mathrm{yr}$ when, self-consistently, it has reached a volume equivalent to the observed Fermi bubbles (see Figure 5); thus, the model hangs together in this parameter region.

\section{NONTHERMAL PARTICLES ACCELERATED AT THE SHOCKS AND THEIR EMISSION}

In the previous section we delimited the range of allowable $\left\{\dot{E}_{0}, \dot{M}\right\}$ parameter space for the GC's giant outflows. In this section we want to determine what our model predicts for the nonthermal emission associated with these outflows, particularly the emission associated with the cosmic-ray electron and cosmic-ray proton and heavier ion populations accelerated at the giant, reverse shocks and subsequently carried into the bubbles' shocked downstream regions (R2) and, if they survive

\footnotetext{
11 At the same time, the CMZ torus has the necessary weight to anchor the Fermi bubble field lines.
} 
long enough, further into the compression shell leading up to the CD (R3). We will determine the hadronic luminosity of the nonthermal protons and heavier ions colliding with gas in R2 and, dominantly, R3, and the radio+microwave synchrotron and $\gamma$-ray IC emission from the nonthermal electrons within each point of the $\left\{\dot{E}_{0}, \dot{M}\right\}$ parameter space. Remarkably, we show below that-within the range of $\dot{E}_{0}$ and $\dot{M}$ allowable according to the analysis of Section 5-our model reproduces the radio, microwave, and $\gamma$-ray nonthermal phenomenology of the giant outflows.

\subsection{Nonthermal Particle Spectra and Energetics}

In the test particle limit (where the dynamics are completely dominated by thermal particles), the spectral index of the nonthermal particle population accelerated at a shock is given by (Bell 1978)

$$
\gamma_{\mathrm{CR}}=\frac{R+2}{R-1}=\frac{(\gamma+1) M^{2}}{(\gamma-1) M^{2}+2},
$$

where $R$ is the shock compression ratio. Observations of $\gamma$-ray and microwave emission reviewed below indicate populations of nonthermal protons and electrons with power-law distributions $d N / d E \propto E^{-\gamma}$ with spectral indices of $\gamma<2.3$ requiring Mach numbers $M \gtrsim 4$. Consistent with this, our modeling favors upstream flows at the giant shocks with Mach numbers in the range 6-9. This will generate nonthermal particle populations with spectral indices of $\sim 2.1$, close to the central values required to explain both the bubbles' $\gamma$-ray and the haze's hard microwave emission.

Energy scales for individual, nonthermal particles of interest to us can be reckoned as follows. Adopting a fiducial magnetic field of $7 \mu \mathrm{G}$ downstream of the shock (Section 6.3.3), the characteristic energy of electrons synchrotron-radiating at $\nu=23 \mathrm{GHz}$ is $E_{\mathrm{c}}=18 \mathrm{GeV}(B / 7 \mu \mathrm{G})^{-1 / 2}(\nu / 23 \mathrm{GHz})^{1 / 2}$. For IC emission, $\gamma$-rays at $E_{\gamma}=1 \mathrm{GeV}$ are upscattered by primary electrons of energy $E_{e} \simeq 640 \mathrm{GeV}\left(E_{\gamma} / 1 \mathrm{GeV}\right)^{1 / 2}\left(E_{\gamma, 0} /\right.$ $\left.6.3 \times 10^{-4} \mathrm{eV}\right)^{-1 / 2}$ adopting an energy for the photon background characteristic of the CMB. Finally, proton primaries of hadronic $\gamma$-rays in the observed range of $1-100 \mathrm{GeV}$ have energies covering the approximate range $10-1000 \mathrm{GeV}$.

These characteristic energies should be compared with upper limits to the energies that the giant shocks might accelerate nonthermal particles. The Hillas criterion that a particle's acceleration time be at least equal to the maximal diffusive escape time from an acceleration region, of fiducial size $r_{\mathrm{acc}} \sim$ $100 \mathrm{pc},{ }^{12}$ sets a maximum energy limit of $E_{\max } \simeq\left(10^{15} \mathrm{eV}\right)$ $\left(r_{\text {acc }} / 100 \mathrm{pc}\right)(B / 7 \mu \mathrm{G})\left(v / 500 \mathrm{~km} \mathrm{~s}^{-1}\right)$. For synchrotron-loss-limited acceleration of electrons over a timescale $t_{\mathrm{acc}} \equiv 4 D / v^{2}$, where $D$ is the diffusion coefficient near the shock and $v$ is a characteristic velocity, in the most optimistic case of Bohm diffusion $D_{\mathrm{Bohm}}=1 / 3 r_{\mathrm{g}} \mathrm{c}$, with $r_{\mathrm{g}}$ the particle gyroradius, we find a maximum upper limit to the accelerated electron energy of $E_{e}^{\max } \simeq\left(4 \times 10^{13} \mathrm{eV}\right)(B / 7 \mu \mathrm{G})^{-1 / 2}\left(v / 500 \mathrm{~km} \mathrm{~s}^{-1}\right)$. In this case, the shocks can easily accelerate electrons up to the requisite energies to produce the observed microwave emission and even the IC $\gamma$-rays by upscattering the CMB (though

\footnotetext{
12 This scale corresponds to the approximate size of linear features found in the $2.3 \mathrm{GHz}$ polarization map that, below, we identify with plausible signatures of the shocks.
}

whether such high-energy electrons can reach the top of the bubbles is another matter).

In our modeling we have explored different values for the fraction, $\epsilon_{\mathrm{CR}}$, of shock mechanical power that is injected into freshly accelerated, nonthermal particles, $L_{\mathrm{CR}}$, finding that ranges of $\sim 5 \%-20 \%$ are acceptable. For the magnetic field amplitudes explored below, we find that electrons are required to take up a fraction $5 \%-30 \%$ of $L_{\mathrm{CR}}$ in order to explain the radio and microwave emission from the bubbles, with the remainder going into ions, predominantly protons.

\subsection{Gamma-Ray Emission from the Bubbles}

\subsubsection{Observations}

The measured $\gamma$-ray spectrum of the bubbles is rather hard, approximately $F_{\gamma} \propto E_{\gamma}^{-2.1}$ between 1 and $100 \mathrm{GeV}(\mathrm{Su}$ et al. 2010), but with a hardening below $\sim 1 \mathrm{GeV}$. The bubbles have a rather flat projected surface brightness ( $\mathrm{Su}$ et al. 2010). A recent paper from the Fermi Collaboration (Ackermann et al. 2014) gives the $100 \mathrm{MeV}-500 \mathrm{GeV}$ luminosity of the bubbles as $\left(4.4 \pm 0.1[\mathrm{stat}]_{-0.9}^{+2.4}[\mathrm{sys}]\right) \times 10^{37} \mathrm{erg} \mathrm{s}^{-1}$. This publication also reports that the bubbles' $\gamma$-ray spectrum cuts off above $\sim 100 \mathrm{GeV}$.

\subsubsection{Expected Hadronic Gamma-Ray Emission}

A successful model of the bubbles' $\gamma$-ray phenomenology needs to explain their luminosity, spectrum, and morphology (and, most generally, spectral morphology). Generic to any hadronic model is a $\sim \mathrm{GeV}$-scale low-energy downturn feature in the spectral energy distribution, whose position in energy is fixed by the mass of the neutral pion. This is broadly consistent with the spectral phenomenology alluded to above (Crocker \& Aharonian 2011).

We show immediately below that our model provides a good match to the overall $\gamma$-ray luminosity of the bubbles. In addition, for acceptable regions of the $\left\{\dot{E}_{0}, \dot{M}\right\}$ parameter space we find moderately high Mach numbers (6-9) at the giant shocks, naturally accounting for the $1-100 \mathrm{GeV}$ hard spectrum of the bubbles (and the hard, nonthermal microwave haze, as we later explain). We also show below that our model predicts that the volumetric emission from the bubbles should peak toward their edges; this is consistent with their flat (projected) surface brightness. We do not explicitly deal with the observed high-energy cutoff here; this may indicate either a high-energy cutoff in the accelerated proton spectrum emerging from the shocks or a loss of confinement of the cosmic-ray hadrons in the bubbles at $\sim \mathrm{TeV}$ energies.

\subsubsection{Hadronic Gamma-Ray Luminosity}

Adapting the calculations presented by Crocker et al. (2014), we can calculate the hadronic luminosity of the bubbles. Our essential argument is that, given the expansion timescale, the evolution of the plasma in the bubbles cannot be treated as adiabatic, but rather the implication of plasma cooling must be considered. This cooling leads to local thermal instability and the formation of overdense, cool condensations that, having lost buoyancy, fall down through the bubbles under gravity. (In the steady-state limit the dropout of these condensations completely saturates the injection rate of fresh plasma mass.) Globally there is a slow flow in the bubbles of material downstream of the reverse shock and toward the $\mathrm{CD}$. As the 
$\mathrm{CD}$ is approached, this flow must be decelerated with a consequent increase in plasma density. Given the $n^{2}$ dependence of the cooling, it is in this overdense shell region (R3 in Figure 1) that thermal instability will preferentially form the condensations. Thus, there will be a flow of gas at a rate (saturating $\dot{M}$ in the steady-state limit) of shocked plasma from the interior volume of the bubbles behind the reverse shocks, $\mathrm{R} 2$, into this shell, R3, with subsequent thermal collapse, and dropout under gravity. We review the observational evidence for formation of cool gas condensations near the edges of the bubbles in Section 7.2.

In our model, in the interior region, R2, the gas evolves adiabatically, whereas in the denser shell, R3, it evolves radiatively. However, a crucial point is that cosmic rays and magnetic fields evolve adiabatically in both shell and volume gas. Adiabatic compression of these nonthermal ISM components thus raises their relative pressure within the shell. In fact, compression of the shell by the plasma in R2 is arrested when the adiabatic energy gain of magnetic fields and cosmic rays results in their reaching pressure equilibrium with the interior plasma. The size of the shell is dually controlled by this and the requirement that the cooling radiation taking place within it (strictly, in the steady-state limit) balances the enthalpy flux represented by the flow of hot plasma from the hot interior.

As we now show, we can use this pressure equilibrium between the adiabatically compressed cosmic rays and magnetic fields in R3 and the thermal plasma in R2 and the size of the shell determined by the enthalpy flux argument to determine the hadronic $\gamma$-ray luminosity of the bubbles. This is dominated by collisions in the shell between the adiabatically compressed cosmic rays and the compressed, cooling gas, which can be calculated as shown below. The Fermi-LAT band hadronic ("pp") luminosity of the shell is

$$
\begin{aligned}
L_{\gamma}^{\mathrm{pp}} & \simeq \frac{3}{2} \times \frac{1}{3} \times f_{\mathrm{bol}} u_{\mathrm{p}}^{\text {shell }} V_{\text {shell }} n_{\mathrm{H}}^{\text {shell }} \sigma_{\mathrm{pp}} \kappa_{\mathrm{pp}} c \\
& =\frac{f_{\text {bol }}}{2} u_{\mathrm{p}}^{\text {shell }} \frac{M_{\text {shell }}}{\mu \mathrm{m}} \frac{n_{\mathrm{H}}^{\text {shell }}}{n_{\text {tot }}^{\text {shell }}} \sigma_{\mathrm{pp}} \kappa_{\mathrm{pp}} c .
\end{aligned}
$$

Here, in the first line, the first $3 / 2$ prefactor (approximately) corrects for the presence of heavy ions among beam and target nuclei (Mori 1997), 1/3 comes from the relative multiplicity of $\pi^{0}$ among all daughter pions, $f_{\mathrm{bol}}$ is the fraction of the bolometric luminosity emitted in the relevant $\gamma$-ray band, $u_{\mathrm{p}}^{\text {shell }}$ is the energy density of relativistic protons in the shell, $\sigma_{\mathrm{pp}} \simeq 4 \times 10^{-26} \mathrm{~cm}^{2}$ is the total hadronic cross section, and $\kappa_{\mathrm{pp}}$ $\simeq 0.5$ is the hadronic inelasticity. In the second line $n_{\mathrm{H}}^{\text {shell }} / n_{\text {tot }}^{\text {shell }}=0.44578$ is the $\mathrm{H}^{+}$to total plasma number density ratio in the shell at solar metallicity, $m$ is the atomic mass unit, and $\mu=0.6039$ is the mean mass of the particles in a solar-metallicity plasma.

We now assume pressure balance between the relativistic shell components ("p" for protons and heavier ions and " $B$ " for the magnetic field), $P_{\mathrm{p}}^{\text {shell }}+P_{B}^{\text {shell }}$, and the thermal plasma in the interior, $P_{\mathrm{FB}}$, as justified above (and also adopt cosmic-ray/ magnetic field equipartition in the shell as justified observationally by Carretti et al. $2013, P_{\mathrm{p}}^{\text {shell }} \simeq P_{B}^{\text {shell }}$ ), so that the pressures represented by these various components are related by

$$
P_{\mathrm{p}}^{\text {shell }} \equiv \frac{1}{3} u_{\mathrm{p}}^{\text {shell }}=\frac{1}{3} u_{B}^{\text {shell }}=\frac{1}{2} P_{\mathrm{FB}} .
$$

We now calculate $M_{\text {shell }}$ as follows: mass conservation implies that the flux of mass from interior volume, of number density $n_{\text {tot }}^{\mathrm{FB}}$, into the shell is equal (in the steady-state limit) to the mass flux into the bubbles, $\dot{M}$. Thus, the effective speed, $v$, with which plasma is carried across the surface, of area $A_{\text {shell }}$, separating the interior region $\mathrm{R} 2$ from the shell $\mathrm{R} 3$ is

$$
\begin{aligned}
v \equiv & \frac{\dot{M}}{\mu \mathrm{m} A_{\text {shell }} n_{\mathrm{tot}}^{\mathrm{FB}}} \simeq 13 \mathrm{~km} \mathrm{~s}^{-1}\left(\frac{\dot{M}}{M_{\odot} \mathrm{yr}^{-1}}\right) \\
& \times\left(\frac{n_{\mathrm{tot}}^{\mathrm{FB}}}{0.002 \mathrm{~cm}^{-3}}\right)^{-1}\left(\frac{A_{\text {shell }}}{2.5 \times 10^{45} \mathrm{~cm}^{2}}\right)^{-1} .
\end{aligned}
$$

The enthalpy flux represented by this flow is dissipated in cooling radiation from the shell; thus,

$$
\frac{5}{2} n_{\mathrm{tot}}^{\mathrm{FB}} k_{B} T_{\mathrm{FB}} v A_{\text {shell }}=\left(n_{\mathrm{tot}}^{\text {shell }}\right)^{2} \Lambda\left[T_{\text {shell }}\right] V_{\text {shell }},
$$

where $\Lambda\left[T_{\text {shell }}\right]$ is the cooling function at the shell temperature and metallicity (the latter assumed to be solar as for the hot plasma) and, geometrically, we assume a thin shell. As for the shell temperature, as we have emphasized, the X-ray spectrum across the edge of the bubbles, encompassing the region of the shell, has been successfully fit with a single-temperature, collisional ionization equilibrium plasma model of temperature $\sim 3.5 \times 10^{6} \mathrm{~K}$, and we therefore adopt $T_{\text {shell }}=3.5 \times 10^{6} \mathrm{~K}$ (so that the temperature in the interior volume is necessarily $>3.5 \times 10^{6} \mathrm{~K}$ ). In reality, the gas distribution in the shell is more complicated as the gas cascades from the high injection temperature, where it starts to cool, down to optical emission line temperatures. Such a multitemperature distribution is inferred, e.g., from the coincident X-ray and $\mathrm{H} \alpha$ emission observed from filaments around the bright central galaxies in clusters (e.g., Fabian et al. 2011) and is common to cooling flows. In any case, here we ignore the complication of the multitemperature nature of the shell gas and assume that the effective temperature apposite to the calculation of shell cooling losses is precisely that found from fitting to the observed X-ray radiation for a single-temperature model.

Rearranging and combining Equations (6.4) and (6.5), we find

$$
\begin{aligned}
V_{\text {shell }} & =\frac{5}{2} \frac{k_{B} T_{\mathrm{FB}} \dot{M}}{\left(n_{\mathrm{tot}}^{\text {shell }}\right)^{2} \Lambda\left[T_{\text {shell }}\right] \mu \mathrm{m}} \\
& =\frac{5}{2} \frac{k_{B} T_{\mathrm{FB}} \dot{M}}{\left(n_{\mathrm{tot}}^{\mathrm{FB}}\right)^{2} \Lambda\left[T_{\text {shell }}\right] \mu \mathrm{m}}\left(\frac{n_{\mathrm{tot}}^{\mathrm{FB}}}{n_{\mathrm{tot}}^{\text {shell }}}\right)^{2} .
\end{aligned}
$$

Following Equation (6.3), specifiying pressure balance between the adiabatically compressed shell magnetic field and cosmic rays and the thermal gas in the interior, and given adiabatic evolution of the cosmic rays,

$$
u_{\mathrm{p}}^{\text {shell }}=\left(\frac{n_{\mathrm{tot}}^{\text {shell }}}{n_{\mathrm{tot}}^{\mathrm{FB}}}\right)^{4 / 3} u_{\mathrm{p}}^{\mathrm{FB}},
$$

then for $u_{\text {therm }}^{\mathrm{FB}}$ as the internal energy density of the plasma in the bubbles, and $L_{\mathrm{CR}}$ and $\dot{E}_{\text {therm }}$ the power fed, respectively, into freshly accelerated cosmic rays and freshly heated plasma 
at the reverse shocks, we have that

$$
\frac{n_{\mathrm{tot}}^{\mathrm{FB}}}{n_{\mathrm{tot}}^{\text {shell }}}=\left(\frac{u_{\mathrm{p}}^{\mathrm{FB}}}{u_{\mathrm{therm}}^{\mathrm{FB}}}\right)^{3 / 4} \simeq\left(\frac{L_{\mathrm{CR}}}{\dot{E}_{\mathrm{therm}}}\right)^{3 / 4} \simeq \epsilon_{\mathrm{CR}}^{3 / 4},
$$

where the last two near equalities rely on adiabatic evolution of both cosmic rays and thermal plasma downstream of the shocks in the interior volume R2. We thus find, implicitly defining the thickness of the shell, $d_{\text {shell }}$ (assumed to be composed of two concentric shells, one around each spherical bubble)

$$
\begin{aligned}
V_{\text {shell }} & \equiv V_{\mathrm{FB}}-2 \frac{4 \pi}{3}\left(r_{\mathrm{FB}}-d_{\text {shell }}\right)^{3} \\
& \simeq \frac{5}{2} \frac{k_{B} T_{\mathrm{FB}} \dot{M}}{\left(n_{\mathrm{tot}}^{\mathrm{FB}}\right)^{2} \Lambda\left[T_{\text {shell }}\right] \mu \mathrm{m}}\left(\frac{L_{\mathrm{CR}}^{\mathrm{FB}}}{\dot{E}_{\mathrm{therm}}^{\mathrm{FB}}}\right)^{3 / 2},
\end{aligned}
$$

which implies a characteristic thickness of the shell in the geometrically thin limit

$$
\begin{aligned}
d_{\text {shell }} \simeq & 510 \mathrm{pc}\left(\frac{T_{\mathrm{FB}}}{6.0 \times 10^{6} \mathrm{~K}}\right)\left(\frac{\dot{M}}{0.1 M_{\odot} \mathrm{yr}^{-1}}\right) \\
& \times\left(\frac{n_{\mathrm{tot}}^{\mathrm{FB}}}{0.004 \mathrm{~cm}^{-3}}\right)^{-2}\left(\frac{L_{\mathrm{CR}}^{\mathrm{FB}}}{\dot{E}_{\mathrm{therm}}^{\mathrm{FB}}} / 0.1\right)^{3 / 2},
\end{aligned}
$$

where we set $A_{\text {shell }}=2.5 \times 10^{45} \mathrm{~cm}^{2}$. The total mass of the shell, in all generality, is

$$
\begin{aligned}
M_{\text {shell }} \simeq & \frac{5}{2} \frac{k_{B} T_{\mathrm{FB}} \dot{M}}{n_{\mathrm{tot}}^{\mathrm{FB}} \Lambda\left[T_{\text {shell }}\right]}\left(\frac{L_{\mathrm{CR}}^{\mathrm{FB}}}{\dot{E}_{\mathrm{therm}}^{\mathrm{FB}}}\right)^{3 / 4} \\
\simeq & 2.5 \times 10^{7} M_{\odot}\left(\frac{T_{\mathrm{FB}}}{6.0 \times 10^{6} \mathrm{~K}}\right)\left(\frac{\dot{M}}{0.1 M_{\odot} \mathrm{yr}^{-1}}\right) \\
& \times\left(\frac{n_{\mathrm{tot}}^{\mathrm{FB}}}{0.004 \mathrm{~cm}^{-3}}\right)^{-1}\left(\frac{L_{\mathrm{CR}}^{\mathrm{FB}}}{\dot{E}_{\mathrm{therm}}^{\mathrm{FB}}} / 0.1\right)^{3 / 4},
\end{aligned}
$$

and we may finally write the $1-100 \mathrm{GeV}$ hadronic luminosity of the shell as

$$
\begin{aligned}
L_{\gamma}^{\mathrm{pp}} \simeq & \frac{15 f_{\mathrm{bol}}}{8} \frac{\left(k_{B} T_{\mathrm{FB}}\right)^{2} \dot{M}}{\mu \mathrm{m} \Lambda\left[T_{\text {shell }}\right]} \frac{n_{\mathrm{H}}}{n_{\mathrm{tot}}} \\
& \times\left(\frac{L_{\mathrm{CR}}^{\mathrm{FB}}}{\dot{E}_{\mathrm{therm}}^{\mathrm{FB}}}\right)^{3 / 4} \sigma_{\mathrm{pp}} \kappa_{\mathrm{pp}} c \\
\simeq & 2.4 \times 10^{37} \mathrm{erg} \mathrm{s}^{-1}\left(\frac{T_{\mathrm{FB}}}{6.0 \times 10^{6} \mathrm{~K}}\right)^{2}\left(\frac{\dot{M}}{0.1 M_{\odot} \mathrm{yr}^{-1}}\right) \\
& \times\left(\frac{L_{\mathrm{CR}}^{\mathrm{FB}}}{\dot{E}_{\mathrm{therm}}^{\mathrm{FB}}} / 0.1\right)^{3 / 4},
\end{aligned}
$$

which compares favorably to the $\sim 2 \times 10^{37} \mathrm{erg} \mathrm{s}^{-1} 1-100 \mathrm{GeV}$ luminosity of the bubbles ( $\mathrm{Su} \&$ Finkbeiner 2012) if $\gtrsim 10 \%$ of the mechanical power at the shock is delivered to cosmic rays. Note that in the interior region, we are normalizing to a somewhat hotter temperature than the $3.5 \times 10^{6} \mathrm{~K}$ measured in
$\mathrm{X}$-rays, assuming that this is characteristic of the cooling shell and that here $f_{\text {bol }} \simeq 0.4$.

The results of a full calculation of the hadronic luminosity of the filaments in the downstream are plotted in Figure 9. Note that there is a subdominant component of the bubbles' hadronic $\gamma$-ray emission due to collisions between the cosmic-ray protons and the rarefied, volume-filling plasma, but, for fiducial numbers, this provides only $5 \%-20 \%$ of the integrated shell luminosity.

\subsubsection{IC Gamma-Ray Emission}

As has been noted, the same population of nonthermal electrons synchrotron-radiating at microwave frequencies to explain the haze may emit IC $\gamma$-rays in the Fermi band with the correct bubble spectrum. Within our scenario, however, we find that this process is subdominant to the hadronic $\gamma$-ray emission explaining $\sim 10 \%-30 \%$ of the total observed $100 \mathrm{MeV}-$ $500 \mathrm{GeV} \gamma$-ray luminosity of the bubbles for the fiducial case that the power fed into freshly accelerated electrons is $10 \%$ of the power fed into all CRs (which is, itself, assumed to be $15 \%$ of the mechanical power at the shock in this figure): $L_{e}=0.1 L_{\mathrm{CR}}=0.1 \times 0.15 \times \dot{E}$; see Figure 9. Of course, this conclusion is contingent on the assumed $L_{e} / L_{\mathrm{CR}}$, but significantly higher values of this ratio can be rejected on two considerations.

1. Within our model, IC cannot reproduce the observed morphology of the bubbles' overall $\gamma$-ray emission alone as the high-energy electrons never reach more than $\sim 300 \mathrm{pc}$ downstream of the shocks (see Figure 10). On the other hand, this expected concentration of IC emission does imply that, within a few degrees of the shocks, it can contribute easily more than half of the total $100 \mathrm{MeV}-500 \mathrm{GeV} \quad \gamma$-ray intensity even for $L_{e} / L_{\mathrm{CR}} \sim 0.1$, and we thus expect $\gamma$-ray substructure around the shocks; preliminarily, we find evidence for such (see Section 7.1.1).

2. Values $L_{e} / L_{\mathrm{CR}} \gtrsim 0.1$ are disfavored on theoretical grounds (Bell 1978), on the basis of analysis of the Galaxy's global cosmic-ray and nonthermal radiation budget (Strong et al. 2010), and from observations and analysis of nonthermal radiation associated with giant cluster shocks (Pinzke et al. 2013; Brunetti \& Jones 2014, and references therein).

\subsubsection{Projected Surface Brightness}

As has been remarked, the bubbles exhibit a rather flat surface brightness. We consider a toy, two-zone geometric model of a spherical shell of luminosity $L_{\text {shell }} \equiv \epsilon_{\text {shell }} V_{\text {shell }}$ enveloping a spherical interior region of luminosity $L_{\text {interior }} \equiv \epsilon_{\text {interior }} V_{\text {interior }}$. The outer radius of the shell is $r_{B}$, and the inner radius of the shell/outer radius of the interior is $r_{\text {shell }}$. In the far field limit, we find from elementary geometric considerations that a roughly flat projected surface brightness for such a two-zone model requires that the volumetric emissivities are related as

$$
\left.\frac{\epsilon_{\text {shell }}}{\epsilon_{\text {interior }}}\right|_{\text {flat }} \simeq\left[1+\left(\frac{r_{B}}{r_{\text {shell }}}\right)^{3}\left(\sqrt{1-\left(\frac{r_{\text {shell }}}{r_{B}}\right)^{2}}-1\right)\right]^{-1}
$$




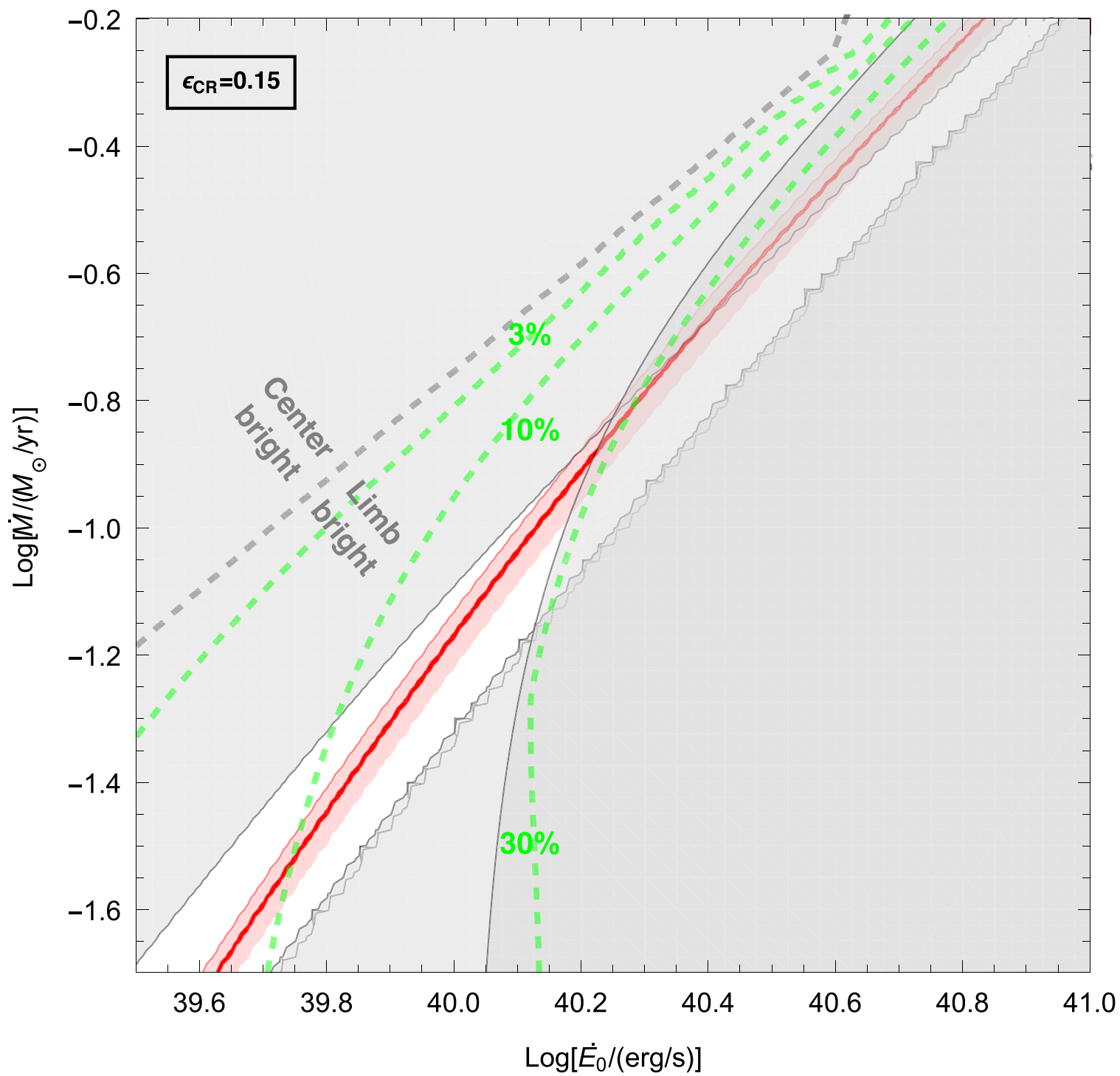

Figure 9. Predicted hadronic and IC $\gamma$-ray luminosity within the allowed parameter space for the case that $P_{\text {halo }}=2 \mathrm{eV} \mathrm{cm}^{-3}, \epsilon_{\mathrm{CR}}=0.15$, and $T_{\text {shell }}=3.5 \times 10^{6} \mathrm{~K}$. Regions that are masked out in gray do not obey all the physical or observational constraints as in Figure 8. The pink/red band is the locus of parameter space points for which the predicted hadronic luminosity is close to that observed (central value of $4.4 \times 10^{37} \mathrm{erg} \mathrm{s}^{-1}$ over $100 \mathrm{MeV}<E_{\gamma}<500 \mathrm{GeV}$ with $1 \sigma$ statistical+systematic errors shown as the pink regions; Ackermann et al. 2014). The green dashed curves report the total $100 \mathrm{MeV}-500 \mathrm{GeV}$ inverse Compton (IC) luminosity as a proportion $\left(3 \%, 10 \%\right.$, or $30 \%$ as labeled) of $4.4 \times 10^{37} \mathrm{erg} \mathrm{s}^{-1}$. The IC curves assume that, of the $15 \%$ of the mechanical energy at each shock that goes into freshly accelerated cosmic rays, $10 \%$ goes into primary electrons, $L_{e}=0.1 L_{\mathrm{CR}}=0.1 \times 0.15 \times \dot{E}$. The gray dashed curve shows where the naive flat surface brightness condition (Equation (6.13)) is exactly satisfied.

This implies the geometric restriction that $r_{\text {shell }}>0.544 r_{B} \sim$ $1.7 \mathrm{kpc}$ (or $d_{\text {shell }} \lesssim 1.4 \mathrm{kpc}$; see orange exclusion region in Figure 8). Over the range $0.544 r_{B}<r_{\text {shell }} \leqslant r_{B}$, one finds $\epsilon_{\text {shell }} /\left.\epsilon_{\text {interior }}\right|_{\text {flat }}>4.3$. In Figure 9 the gray dashed curve shows where Equation (6.13) is satisfied exactly; left and upward of the curve, the overall $\gamma$-ray surface brightness morphology tends to center brightened, and right and downward of the curve, the morphology tends to limb brightened according to our simplistic analysis. A constant-volume emissivity model would produce a center-brightened morphology, and the original instantiation of the hadronic model (Crocker \& Aharonian 2011) was criticized on this ground. The updated hadronic model presented here, if anything, tends toward limb brightening in the favored parameter space according to Equation (6.13). However, we do not believe that this is a problem for our model for three reasons: (i) the observed surface brightness is not exactly flat, indeed there is, e.g., a large region close to the east edge of the southern bubble that appears brightened ("the cocoon"; Su \& Finkbeiner 2012; Ackermann et al. 2014); (ii) we have assumed an unrealistically simple geometry in deriving Equation (6.13); and (iii) we expect, in reality, some relative diminution of shell hadronic emission with a compensating increase in interior emission due to condensations falling down through the bubbles under gravity, still emitting $\gamma$-rays (see Figure 1). A more accurate treatment of this geometrical aspect of the problem requires numerical modeling.

\subsection{Synchrotron Emission \\ 6.3.1. Observations}

The $23-44 \mathrm{GHz}$ microwave haze is found (Finkbeiner 2004; Dobler \& Finkbeiner 2008; Dobler 2012a; Ade et al. 2013) in 


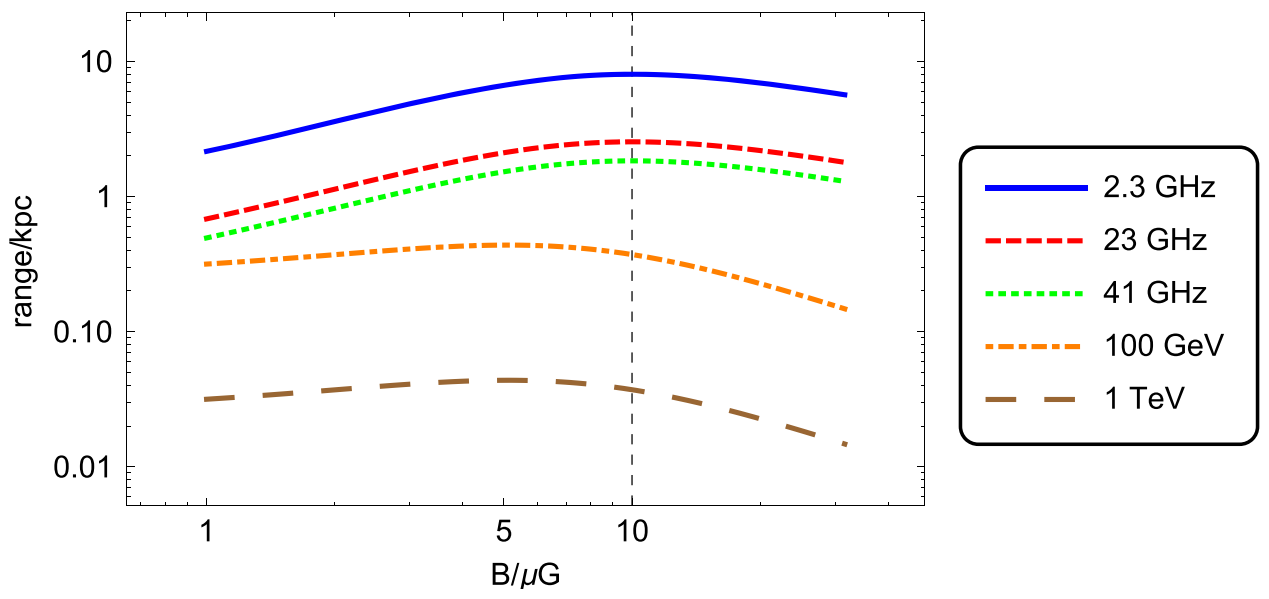

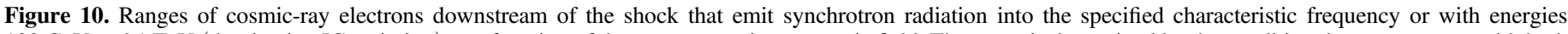

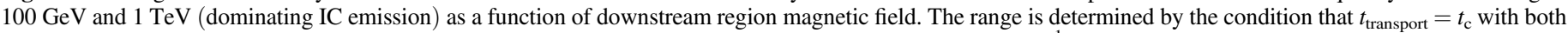

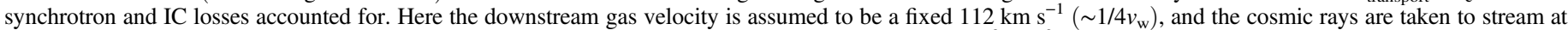

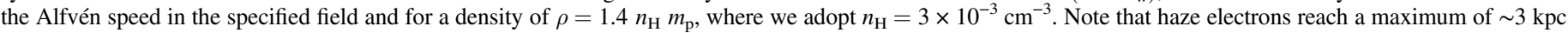

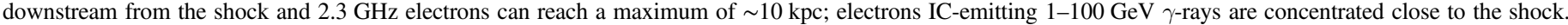

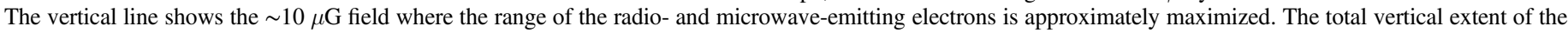

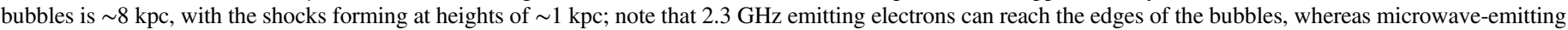

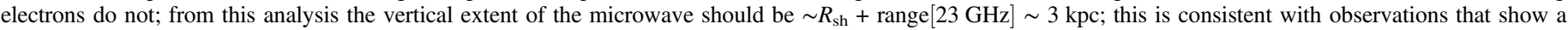
drop-off in the intensity of the microwave emission at $\sim 35^{\circ}$, corresponding to the distance of $2.7 \mathrm{kpc}$ for the near surface of the bubbles at $5 \mathrm{kpc}$ distance.

total-intensity data up to Galactic latitudes $|b| \lesssim 35^{\circ}$, above which it becomes severely attenuated, though there has been a claim (Dobler 2012b) that faint, haze-like emission is detected up to the latitudinal extremities of the bubbles. Here we adopt $0.4 \mathrm{sr}$ for the solid angle of the haze emission. The haze has a hard spectrum $F_{\nu} \propto \nu^{-0.55}$ (Ade et al. 2013), characteristic of synchrotron emission from a population of electrons with spectrum $d N_{e} / d E_{e} \propto E_{e}^{-2.1}$ freshly accelerated at a strong shock.

It has also been claimed (Gold et al. 2011) that the haze exhibits little coincident polarized emission. On the other hand, regions of enhanced, polarized emission coincident with substructure within the bubbles have actually been found, in particular, a bright region coincident with part of the eastern edge of the north bubble (Jones et al. 2012) and the counterparts of the strongly magnetized ridges found in polarized intensity at $2.3 \mathrm{GHz}$. Indeed, there is diffuse, polarized $23 \mathrm{GHz}$ emission evident over most of the solid angle of the bubbles (see Figure 3 of Carretti et al. 2013).

The polarization ridges appear to wrap around the near surface of the roughly biconical structure circumscribing the bubbles in a fashion consistent with the rotation of the Galaxy. There is diffuse, polarized $2.3 \mathrm{GHz}$ emission enveloping the entire bubbles, stretching beyond them, in fact, at high latitudes in two plumes that extend toward Galactic northwest and southwest. In contrast to the haze detected in total intensity at microwave frequencies, the spectrum of polarized intensity between 2.3 and $23 \mathrm{GHz}$ is steep, $F_{\nu} \propto \nu^{-1.1}$ within the solid angle of the bubbles (Carretti et al. 2013), consistent with an electron population of mixed ages that has been cooled by synchrotron and/or IC emission; beyond the bubbles and extending into the plumes, the spectrum further steepens (see Figure S4 of the supplementary material of Carretti et al. 2013).

\subsubsection{Synchrotron Emission in Our Model}

Before proceeding to detailed discussion, we explain briefly how our model accounts for the complicated synchrotron emission phenomenology of the GC's giant outflows. For clarity, note that in general we shall refer to the $23-44 \mathrm{GHz}$ band as "microwave" and $2.3 \mathrm{GHz}$ emission as "radio."

The nucleus directly accelerates a population of hardspectrum primary electrons that is advected from the region before it can lose much energy radiatively in situ (Crocker et al. 2011a, 2011b; Crocker 2012; Carretti et al. 2013). These electrons cool adiabatically and radiatively in the expanding outflow (R1) until they encounter the giant reverse shock in either bubble where they are reaccelerated.

At latitudes in the bubbles higher than the shocks, there are then effectively three zones for synchrotron emission in our model.

1. A zone downstream of each reverse shock, partially encompassing R2, where the electrons are being conveyed systematically downstream by the combination of the downstream flow and their streaming along magnetic field lines. In this zone, there is a one-to-one correspondence between distance from the shock and electron "age." The frequency-dependent extent of this region is given approximately by the requirement that the transport time from the shock be less than the total (synchrotron+IC) cosmic-ray electron loss time. The synchrotron spectrum within this zone is thus "uncooled," $F_{\nu} \sim \nu^{-0.5}$, reflecting that the electron spectrum itself is simply the hard injection spectrum produced at the shock, $d N_{e} / d E_{e} \sim E_{e}^{-2}$, up to some (position- and frequencydependent) maximum energy above which it is exponentially cut off (see Figures 10 and 11). The hard-spectrum microwave haze is largely attributable to the synchrotron radiation from this electron population that is radiating on the volume-filling magnetic field in the base of R2.

2. A zone corresponding to the compression shell that covers, in projection, the entire solid angle of the $\gamma$-ray bubbles out to the $\mathrm{CD}$, i.e., R3. In this zone, the adiabatically amplified magnetic field pervading the cooling gas is rather strong and regular. Synchrotron 


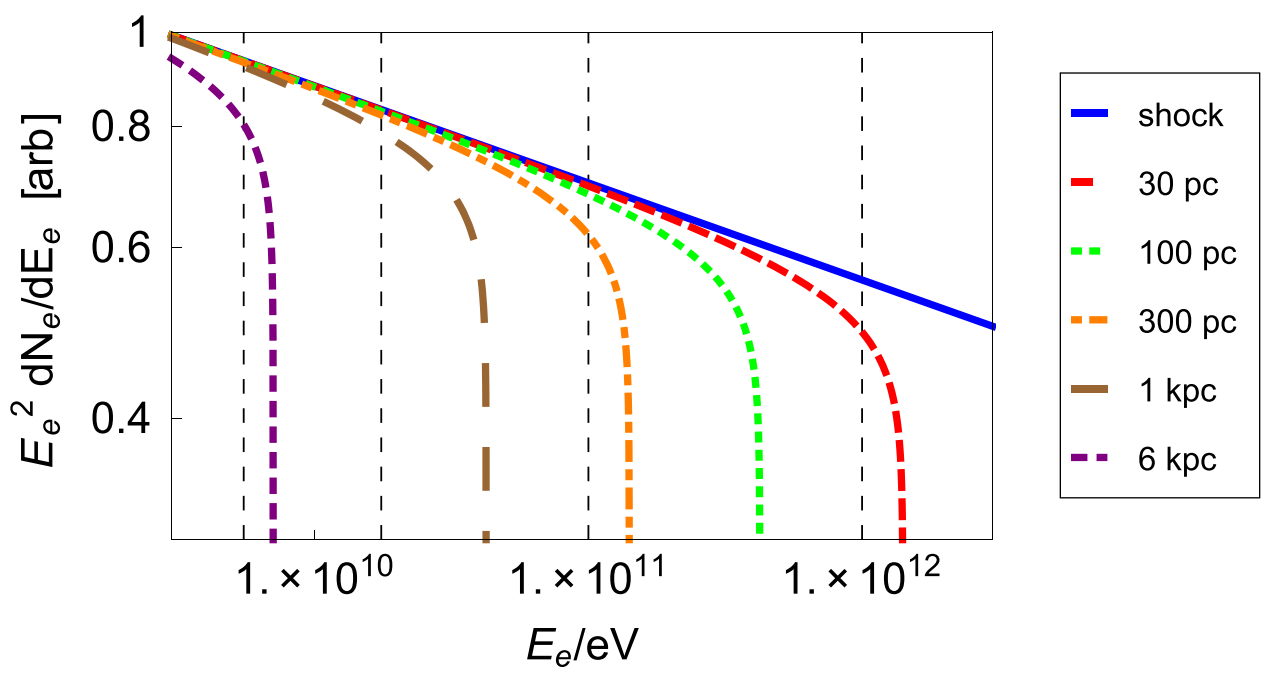

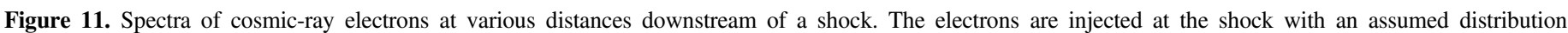

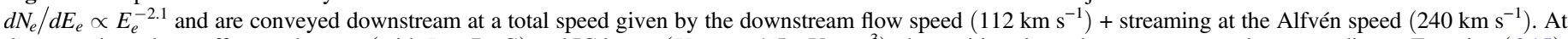

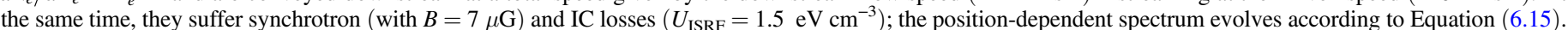

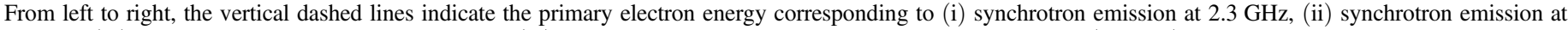

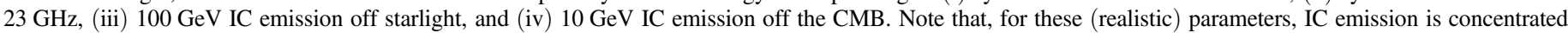

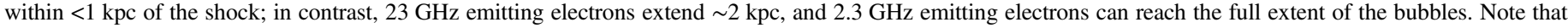

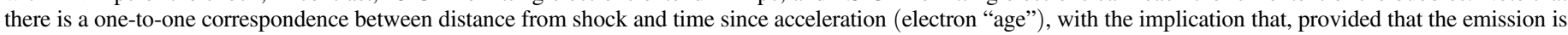

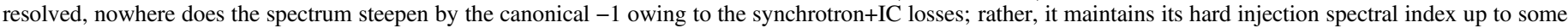
distance/time-dependent energy above which it is cut off.

emission here is by (i) the adiabatically compressed, lower-energy primary electrons that have long enough loss time that they can travel all the way from the shock and (ii) secondary electrons that result from hadronic collisions occurring in the denser shell gas.

Two important considerations for synchrotron emission from R3 are that (i) given relative transport and loss times microwave-emitting primary electrons accelerated at the shock never reach this zone; and (ii) in this zone, there is a mixture of electrons of different ages as all shell electrons simply cool in situ (with the qualification that a fraction of the electrons apparently leak from R3 into the radio plumes, R4; see below). The latter implies that, above some break energy, $E_{\mathrm{bk}}$, the integrated, steady-state shell electron spectrum of $\mathrm{R} 3$ is $d N_{e} / d E_{e} \sim E_{e}^{-3}$ (Kardashev 1962), implying a synchrotron spectrum above $\nu_{\text {bk }}$ of $F_{\nu} \sim \nu^{-1}$. This explains the steep, polarized, and rather uniform spectrum measured between 2.3 and $23 \mathrm{GHz}$ over the solid angle of the radio lobes. The break energy is implicitly defined by $t_{\mathrm{c}}\left(E_{\mathrm{bk}}\right)=t_{\mathrm{FB}}$, where $t_{\mathrm{c}}$ is the energy-dependent electron cooling time (with cooling processes dominantly synchrotron radiation and IC for the relevant electron energy range) and $t_{\mathrm{FB}}$ is the age of the bubbles over which the electrons have been accumulated in the shell. At even higher energies (for typical parameters) the spectrum suffers a further quasiexponential cutoff owing to the fact that higher-energy primaries can never reach this zone; this cutoff is softened by the presence of secondary electrons produced in situ, which can supply microwaves even at these large distances from the shock (see Dobler 2012b).

3. A zone identified with the polarized radio plumes that extend beyond the boundaries of the north and south bubbles at high latitudes. For this zone, a one-to-one correspondence between distance from the bubbles' edges (the $\mathrm{CD}$ ) and electron age can be reestablished as the electrons are systematically advected into the plumes. We thus expect, in principle, that the position-dependent electron distribution takes the form of the injection spectrum (already steepened to $\sim E_{e}^{-3}$ ), with an exponential cutoff that moves to progressively lower electron energy with increasing distance from the edges. With only two spectral data points currently available (at 2.3 and $23 \mathrm{GHz}$ ), this cutoff translates into a steepening with distance into the plumes (matching observations).

\subsubsection{Magnetic Fields}

To determine the intensity of synchrotron emission from the bubbles, we need to know the magnetic field amplitude in two environments: in the volume-filling plasma phase interior to the bubbles, R2 in Figure 1, and in the denser shell formed by local thermal instability, R3. For the latter, as mentioned, we assume that the pressure of the adiabatically compressed, nonthermal ISM components, i.e., cosmic rays + magnetic fields, reaches equilibrium with the plasma pressure in the interior, $P_{\mathrm{FB}}=2.0 \mathrm{eV} \mathrm{cm}^{-3}$ for fiducial parameters. Given the finding (Carretti et al. 2013) that CRs and magnetic fields are in rough equipartition in the bubbles (and, therefore, in the shell adopting an adiabatic index for the tangled magnetic fields of $\left.\gamma_{B}=4 / 3=\gamma_{\mathrm{CR}}\right)$, then $U_{B}^{\text {shell }} \simeq 3 / 2 P_{\mathrm{FB}} \simeq 3 \mathrm{eV} \mathrm{cm}^{-3}$ so that $B_{\text {shell }} \simeq 11 \mu \mathrm{G}$. This is in good agreement with the magnetic field amplitude of $\sim 12 \mu \mathrm{G}$ suggested by an equipartition analysis of the radio data (and supported by an independent broadband analysis that does not assume equipartition; Carretti et al. 2013) to pertain in a 200-300 pc deep "sheath" enveloping the bubbles.

With this determination, we may work backward to arrive at the adiabatically "decompressed" field pertaining in the R2 plasma, $B_{\mathrm{FB}}=\left(n_{\mathrm{FB}} / n_{\mathrm{fil}}\right)^{2 / 3} B_{\mathrm{fil}} \simeq\left(L_{\mathrm{CR}} / \dot{E}_{\mathrm{cool}}\right) B_{\mathrm{fil}} \sim 5-7 \mu \mathrm{G}$. 
Again, this is consistent with a determination for the volumefilling field amplitude made on the basis of an equipartition analysis of $2.3 \mathrm{GHz}$ radio polarization data (Carretti et al. 2013).

\subsubsection{Synchrotron Emission from the Shock Downstream}

Cosmic-ray transport downstream of the shock is a combination of the $\sim 100-150 \mathrm{~km} \mathrm{~s}^{-1}$ flow of the shocked, downstream plasma and cosmic-ray streaming along the field lines at the Alfvén velocity (Kulsrud \& Pearce 1969; Skilling 1971) of $\sim 240 \mathrm{~km} \mathrm{~s}^{-1}$. The range microwave-emitting electrons can reach in their loss time is then a function of magnetic field amplitude, which controls both synchrotron losses and the Alfvén velocity and reaches a maximum for $B \simeq$ $10 \mu \mathrm{G}$; see Figure 10. Here the cooling time, $t_{\mathrm{c}}$, is the time taken for an electron injected at the accelerator with the maximal possible energy, $E_{\max }$, to be cooled to the energy at which it synchrotron-radiates into the frequency range of concern, $E_{\mathrm{c}} \equiv m_{e} c^{2} \sqrt{\nu / \nu_{\mathrm{g}}[B]}$, where $\nu_{\mathrm{g}}[B]$ is the electron gyroradius in magnetic field $B$. The energy of an electron injected at $E_{0}$ into a constant magnetic field $B$ evolves according to

$$
E[t]=\frac{E_{0}}{1+t / \tau\left[E_{0}\right]},
$$

where $\tau[E]=1.3 \times 10^{8}(B / 10 \mu \mathrm{G})^{-2}(E / \mathrm{GeV})^{-1} \mathrm{yr}$, ignoring non-synchrotron losses (particularly IC) for illustrative purposes and assuming pitch-angle scattering on timescales short with respect to the loss times (e.g., Reynolds 2009).

With this description of the energy evolution of the electrons, one obtains that an electron spectrum injected as a power law by the accelerator, $d N_{e}\left[E_{0}\right] / d E \equiv K E_{0}^{-s}$, evolves according to

$$
\frac{d N_{e}[E, t]}{d E}=K E^{-s}(1-t / \tau[E])^{s-2} .
$$

The evolution of a cosmic-ray electron spectrum, injected with $s=2.1$, as the electrons are conveyed downstream of a shock and, simultaneously, suffer synchrotron+IC losses is illustrated in Figure 11.

Once the transport time exceeds the cooling time $t_{\mathrm{c}}$, a cutoff feature is introduced into the electron spectrum. Applied to the outflow population, $\mathrm{GeV}$ IC-emitting electrons only reach a few $\times 10^{2}$ pc past the shocks and microwave-emitting electrons reach a few kpc.

\subsubsection{Synchrotron Emission from the Compression Shell}

In contrast, lower-energy $\sim \mathrm{GHz}$ emitting electrons survive longer, reach the shell before the $\mathrm{CD}$, and are accumulated inside it (see Figure 10). Compression of the cooling plasma in this shell leads to adiabatic amplification of the frozen-in magnetic field and cosmic rays, implying a second reenergization of those electrons that actually survive to this point. The spatially coincident, enhanced gas and cosmic-ray ion populations close to the bubbles' edges or in the filaments also lead, however, to significant hadronic collisions, which, in addition to producing hadronic $\gamma$-ray emission, also inject secondary electrons (and positrons) via the decay of collisionally produced charged mesons. The edges/filaments are thus populated by both enhanced primary and secondary electron populations in addition to adiabatically compressed magnetic fields. The electrons do not escape from these regions but lose their energy in situ to synchrotron radiation. Accumulation and trapping of electrons in this region lead to mixing of cosmicray electron populations of various ages. Cooling + mixing (in projection) of differently aged electron populations will be enhanced if there are downdrafts toward the edges of the bubbles or convection within it. Mixing steepens the overall steady-state population into an $\propto E_{e}^{-3}$-type distribution (Kardashev 1962). The steep-spectrum, polarized emission detected at $2.3 \mathrm{GHz}$ (Carretti et al. 2013) is largely attributable to this population that radiates in the enhanced magnetic field region associated with the cooling shell toward the edges of the bubbles (and possibly also swept-up halo magnetic field outside the CD). We can thus evaluate the $2.3 \mathrm{GHz}$ emission from the bubbles in a thick-target, single-zone model; the emission thus calculated is displayed in Figure 12 (green curves).

A prediction of our model is the existence of a break in the shell radio spectrum corresponding to where the synchrotron $+\mathrm{IC}$ cooling of shell electrons becomes less than the age over which they have been accumulated, roughly the age of the bubbles $t_{\mathrm{FB}}$. The break occurs at a frequency of

$$
\nu_{\mathrm{br}}=1.96 \times 10^{8} \mathrm{~Hz}\left(\frac{B}{10 \mu \mathrm{G}}\right)^{-3}\left(\frac{t_{\mathrm{FB}}}{10^{8} \text { years }}\right)^{-2} .
$$

The fact that the $23-2.3 \mathrm{GHz}$ spectrum of the radio lobes is $\propto \nu^{-1.1}$ with the break necessarily below $2.3 \mathrm{GHz}$ implies corroborative evidence that the outflow structures have an age of $>\left(2.9 \times 10^{7} \mathrm{yr}\right)\left[B_{\text {shell }} /(10 \mu \mathrm{G})\right]^{-3 / 2}\left(\nu_{\mathrm{br}} / 2.3 \mathrm{GHz}\right)^{-1 / 2}$.

\subsubsection{Synchrotron Emission from the Radio Plumes}

Within the solid angle of the cooling shell that covers the bubbles, mixing of electrons of different ages implies a rather uniform, cooled spectrum. Note, however, that if some lowenergy electrons are able to escape from the bubbles entirely, leaking out in a systematic flow, a position-dependent steepening can be reestablished. This expectation is borne out by the radio+microwave phenomenology: the spectrum of polarized intensity between 2.3 and $23 \mathrm{GHz}$ is rather constant at $F_{\nu} \propto \nu^{-1.1}$ within the solid angle of the bubbles (accounting for depolarization effects at $2.3 \mathrm{GHz}$ close to the plane) but steepens going into the radio plumes that extend beyond the edges of the bubbles.

\subsubsection{Energetics of Synchrotron Emission and Secondary Electrons}

For the magnetic field amplitudes investigated, our calculations of synchrotron emission show that a power going into freshly accelerated electrons of 5\%-20\% the total cosmic-ray power at the shock is required to reproduce the observed radio and microwave emission, i.e., $L_{e} \simeq(0.05-0.2) \times L_{\mathrm{CR}}$. Here $L_{\mathrm{CR}}$, the power going into all cosmic rays at the shock, is assumed to be a fixed fraction of $15 \%$ of the total mechanical power available at the shock. Figure 12 shows the $2.3 \mathrm{GHz}$ and microwave synchrotron emission of both primary and secondary electrons. Within our favored parameter space, secondary electrons generated in the filaments explain 5\%-20\% of the bubbles' $2.3 \mathrm{GHz}$ luminosity. 


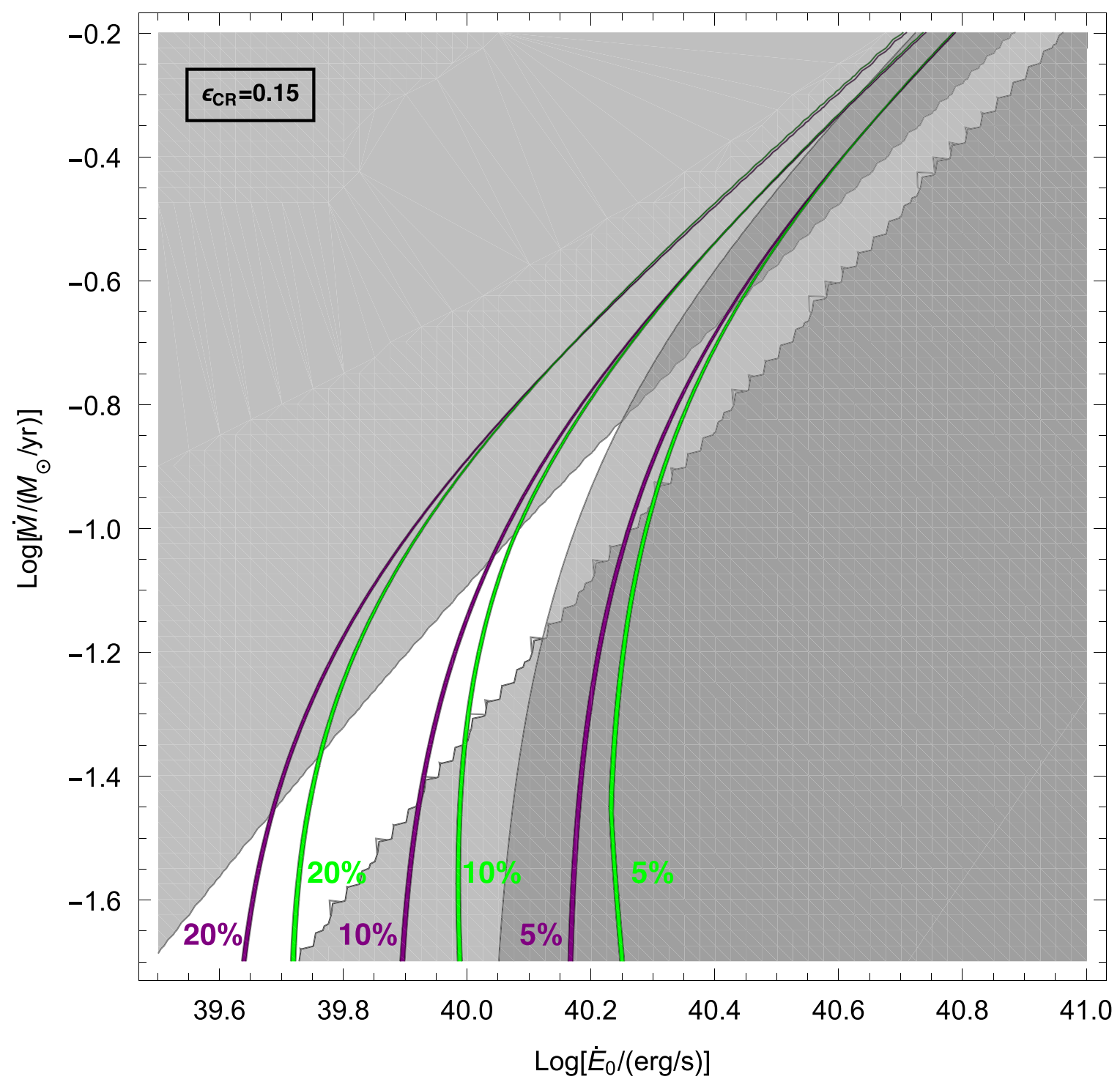

Figure 12. Predicted synchrotron emission for $P_{\text {halo }}=2 \mathrm{eV} \mathrm{cm}^{-3}, \epsilon_{\mathrm{CR}}=0.15$, and $T_{\text {shell }}=3.5 \times 10^{6} \mathrm{~K}$. Regions masked out in gray do not obey all the physical constraints as described in Figure 8. Purple curves refer to the microwave haze and green curves to the giant, $2.3 \mathrm{GHz}$ radio lobes. The curves are labeled according to where the modeled 2.3 and $23 \mathrm{GHz}$ intensity matches that observed for the nominated percentage of total cosmic-ray power going into freshly accelerated electrons, viz., $L_{e}=\{5 \%, 10 \%, 20 \%\} \times L_{\mathrm{CR}}$, where $L_{\mathrm{CR}} \equiv \epsilon_{\mathrm{CR}} E$, with $\dot{E}$ the mechanical power arriving at the shocks. The curves account for both primary and secondary electron synchrotron; here secondary synchrotron accounts for $5 \%-20 \%$ of the total $2.3 \mathrm{GHz}$ intensity over the favored parameter space. The purple curve for emission over the haze region assumes a volume-filling magnetic field of $7 \mu \mathrm{G}$ and an interstellar radiation field calculated at $3 \mathrm{kpc}$ above the plane $\left(U_{\text {ISRF }}=1.7 \mathrm{eV} \mathrm{cm}^{-3}\right.$; Porter et al. 2008); we adopt reference values for the haze intensity at $23 \mathrm{GHz}$ of $1200 \mathrm{Jy} \mathrm{sr}^{-1}$ (Su et al. 2010) and $18.5 \mathrm{kJy} \mathrm{sr}^{-1}$ for the total intensity at $2.3 \mathrm{GHz}$ (Carretti et al. 2013).

\section{CONNECTIONS TO OBSERVATION}

\subsection{Observational Evidence for Giant Shocks in the Bubbles}

\subsubsection{South Bubble}

The S-PASS $2.3 \mathrm{GHz}$ polarization observations reveal a large-scale, linear depolarization structure (strictly, a region of low polarization intensity) in the southern bubble. The feature extends in a southeast direction from the vicinity of the western edge of the south bubble at $(l, b) \sim\left(350^{\circ},-17^{\circ}\right)$ to the vicinity of the eastern edge of this bubble at $\sim\left(9^{\circ},-32^{\circ}\right)$ (see the supplementary material of Carretti et al. 2013, Section 4.1 and Figure S6). There are counterparts to this feature evident in $\gamma$ rays (where the feature appears as an edge particularly evident in 10-30 GeV Fermi data; see de Boer \& Weber 2014, Figure 3 (a); see also R. Yang 2015, private communication) and in $\mathrm{H} \alpha$ emission. ${ }^{13}$ At $2.3 \mathrm{GHz}$, this feature is likely the result of cancellation between intrinsically polarized synchrotron emission on two perpendicular, line-of-sight-superposed magnetic field structures. One of these is the front-side sheath of the south bubble, whose gross magnetic field structure extends in a roughly southwest direction, as evident from the 2.3 and $23 \mathrm{GHz}$ polarization data (reflecting the rotation of the bubble base in the same sense as the Galaxy and conservation of angular momentum in the expanding outflow; Carretti et al. 2013). The cancelling field must run southeast along the long axis of the depolarization feature.

$\overline{13}$ A. Hill (2014, private communication). 


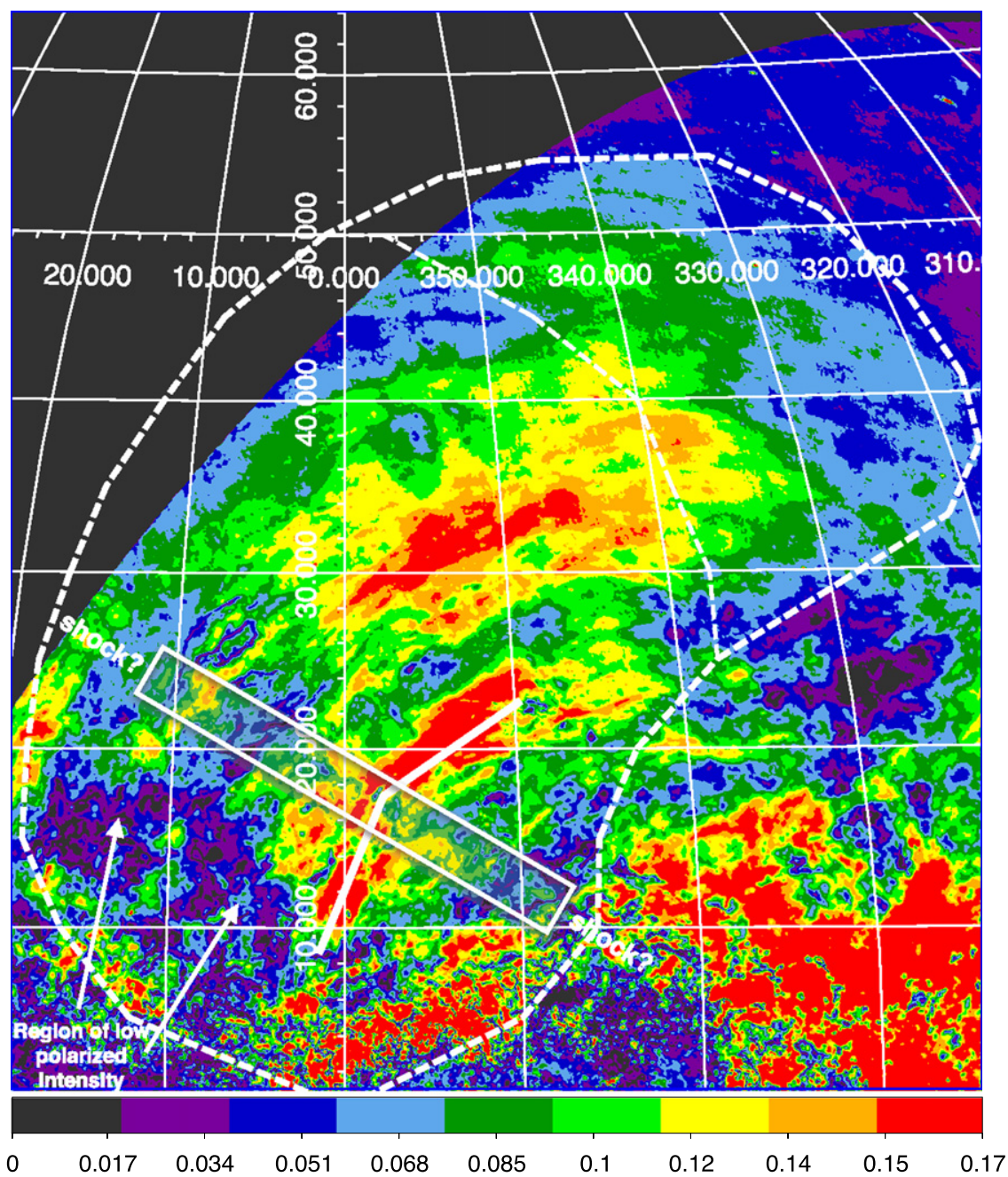

Figure 13. Inflexion in the GCS in the northern bubble traced in white. Colors show $2.3 \mathrm{GHz}$ polarization data from S-PASS (Carretti et al. 2013) in Galactic coordinates with east to the left and the edge of the black triangle to upper left corresponding to the instrumental horizon. The color bar units are Jy/beam (convolved beam is approximately $10^{\prime}$ ). The outer dashed contour delimits the northern S-PASS polarized radio lobe (Carretti et al. 2013); the northern Fermi bubble (Su et al. 2010) edge is contiguous except in the northwest, where it runs inside the boundary of the radio lobe, as shown by the thinner dashed line. The large, inclined rectangle indicates the position of a faint depolarization feature, running from northeast (top left) to southwest (bottom right) through the inflexion point, which might be attributed to a magnetic field aligned with the surface of the giant reverse shock. A region of low polarized intensity at $2.3 \mathrm{GHz}$ in the lower southeast is indicated; this region is also evident at $23 \mathrm{GHz}$.

We believe that a compelling explanation of this feature is that it represents the alignment of magnetic field lines with the shock front in the southern bubble (i.e., there is a perpendicular magnetic field configuration at the shock). Adopting a rough distance to the near side of the bubble of $5 \mathrm{kpc}$, the $z$-height of the depolarization feature is $1-3 \mathrm{kpc}$, similar to the shock location suggested by our analysis in Section 3 .

\subsubsection{North Bubble}

In the northern bubble there is only a faint feature possibly corresponding to the depolarization feature described above (see Figure 13). The north, however, is highly confused and suffers from depolarization at $2.3 \mathrm{GHz}$ significantly off the plane owing to $\rho$ Ophiucus and other local, high-latitude gas structures. There is, however, a feature of the northern bubble that is highly suggestive of a shock. The Galactic Center Spur (GCS; Sofue et al. 1989; Carretti et al. 2013; see Figure 13) is a highly collimated total- and polarized-intensity radio continuum feature that extends in a northwest direction from just east of the Galactic center to Galactic latitudes of $b \sim 25^{\circ}$.
Carretti et al. (2013) have claimed that this feature wraps around the forward surface of the northern bubble, an interpretation we adhere to.

On the basis of a geometric analysis of the overall curvature of this structure, Carretti et al. (2013) also claimed, however, that the spur provides evidence that gas is being driven aloft into the bubbles at a speed of $\sim 1000 \mathrm{~km} \mathrm{~s}^{-1}$ up to heights of at least a few kpc. This analysis was supported by an independent argument that if the $2.3 \mathrm{GHz}$ emitting electrons were accelerated (exclusively) in the nucleus, they would have to be transported at a similar speed in order for them to reach the top of the bubbles in their loss time. This high velocity over the entire bubbles is not consistent with our present model, which suggests that while gas may be injected into the base of the bubbles at $\gtrsim 500 \mathrm{~km} \mathrm{~s}^{-1}$, it is slowed to $100-150 \mathrm{~km} \mathrm{~s}^{-1}$ upon encountering the giant shocks at heights of $\sim 1 \mathrm{kpc}$.

Moreover, the assumptions leading up to the estimated $\sim 1000 \mathrm{~km} \mathrm{~s}^{-1}$ presented by Carretti et al. (2013) are no longer met in our revised scenario. In particular, we now expect injection of high-energy electrons at large scales in the bubbles 


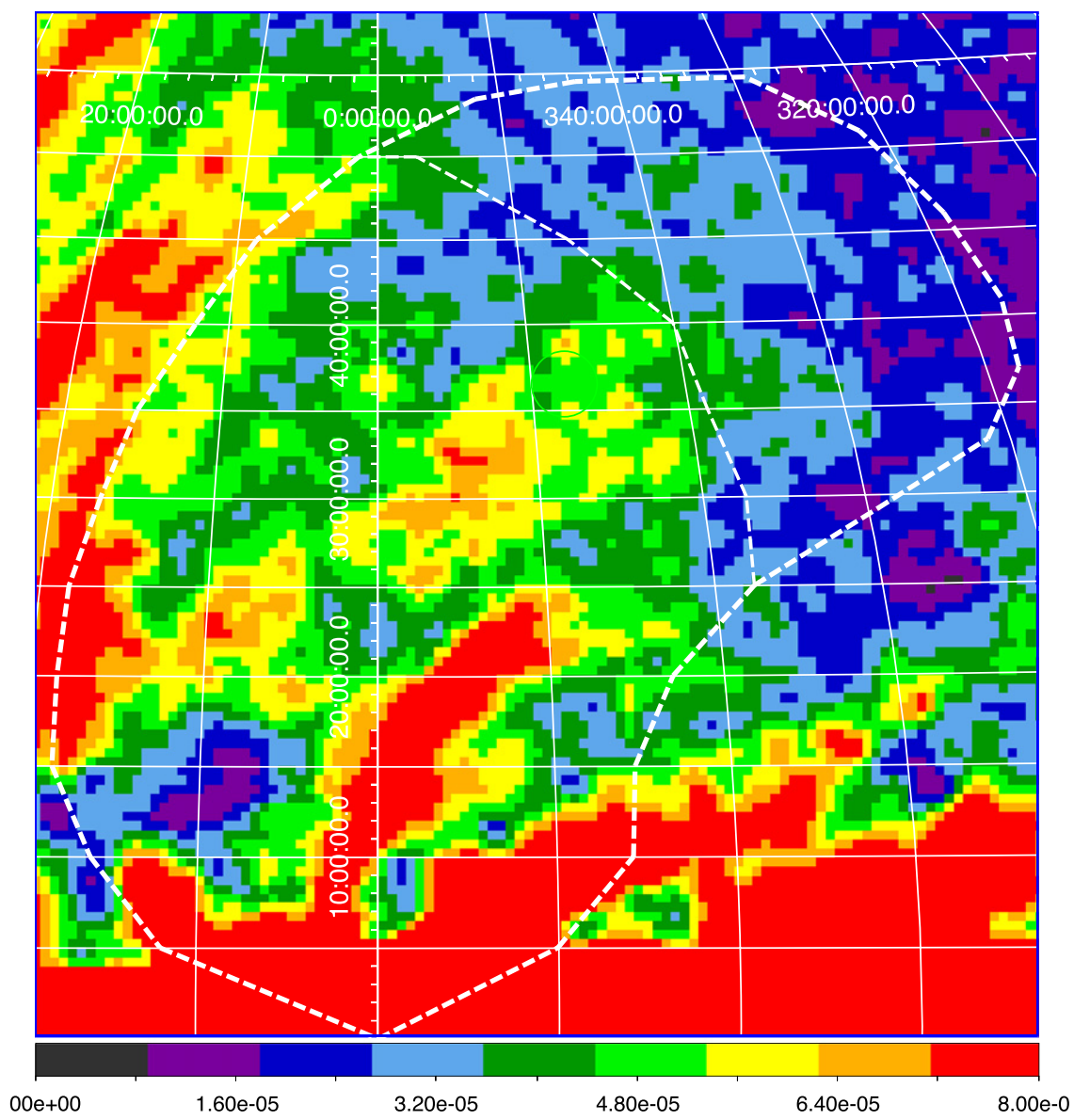

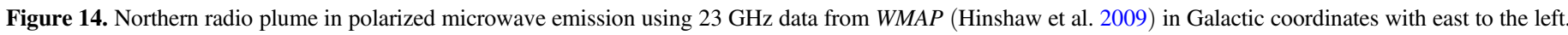

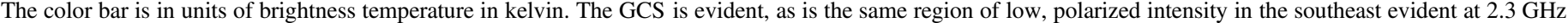
that we suspect is below the latitude of the reverse shock.

either via direct acceleration at the large-scale shocks or as secondaries. The shock in the north bubble changes our expectation for the geometry of the GCS. In fact, we expect an inflexion point in the GCS at the shock as the vertical speed of the flow is slowed by $\sim 1 / 4$ crossing the shock. Such an inflexion does seem to be present in the GCS, and it has the correct geometry: at $b \sim 17^{\circ}$ the angle between the long axis through the GCS changes from $\sim 17^{\circ}$ to $\sim 48^{\circ}$ (see Figure 13), suggesting a change of speed $\sim \tan \left[17^{\circ}\right] / \tan \left[48^{\circ}\right]=0.27$, consistent with a strong shock.

Furthermore, an independent determination (Vidal et al. 2014) of the spectral index of the polarized emission between 23 and $33 \mathrm{GHz}$ from the GCS reveals a hardening at higher latitudes for the radio spectral index $\left(b \gtrsim 18^{\circ}\right)$ from 1.52 \pm 0.51 to $0.62 \pm 0.44$ (with a similar hardening in a region coincident with part of the eastern edge of the north bubble), consistent with a giant shock - at the correct height above the plane-reaccelerating electrons, as we have described above.

Another piece of corroborative evidence in support of this interpretation of the shock geometry in the north is the following: consideration of Figure 13 shows that the southeast region of the north bubble is comparatively dim in $2.3 \mathrm{GHz}$ polarized emission. While, in general, depolarization due to magnetized plasma along the line of sight may affect emission up to latitudes of $\sim 10^{\circ}$, this region extends to $\sim 20^{\circ}$ close to the eastern edge. Moreover, the very same depolarization region is evident also at $23 \mathrm{GHz}$ (see Figure 14), a frequency high enough to be completely unaffected by Faraday depolarization in this region of the sky. We expect, therefore, that this lack of detected polarized emission betokens a lack of intrinsic emission along this line of sight. Significantly, this dim region is below the putative shock feature. This can be naturally explained if, on the one hand, the high-energy primary electrons requisite to produce the emission do not reach these heights from the nucleus and, on the other hand, are only reaccelerated to microwave-emission energies at the shock that is further from the nucleus.

\subsubsection{Why Are the Putative Shock Features Not Parallel to the Plane?}

Of course, our scenario does not explain why the reverse shock surfaces fail to parallel the Galactic plane, but two interesting (and not incompatible) explanations for this phenomenology present themselves.

1. As already remarked, the long axes of the bubbles do not run perpendicular to the plane in either hemisphere but rather appear to be pushed toward Galactic west; the radio polarization data accentuate this impression, with long plumes of emission extending beyond the edge of the $\gamma$ ray bubbles at high latitudes toward Galactic northwest and southwest. Whatever the origin for this apparent distortion, the southern and northern shocks seem to run more or less perpendicular to the inferred flow. 
2. Currently, star formation activity in the Galactic center is concentrated east of $l=0$; there is a preponderance of molecular gas on this side of the region, and Sagittarius $\mathrm{B} 2$, one of the Galaxy's most active star-forming giant molecular clouds, resides there (Morris \& Serabyn 1996). Moreover, of the three large stellar clusters in the region, one surrounds the supermassive black hole, but the other two, Quintuplet and Arches, are located to the east side. The Quintuplet, at $\sim 4 \mathrm{Myr}$ old, is likely to have already hosted a number of very massive core-collapse $\mathrm{SNe}$, and there is tantalizing evidence that some such $\mathrm{SNe}$ have occurred (Sofue 2003). So it may well be that, even if, on average, injection of mechanical energy is evenly distributed east and west of the GC, it is now predominantly coming from the east, leading to a localized increase in the outflow velocity that tends to push the shocks further into the halo on this side. This localization of the mechanical energy injection associated with star formation may be the origin of the ridge features evident from polarization data on the forward surfaces of both north and south bubbles (Carretti et al. 2013).

We will provide a more detailed and quantitative analysis of this putative shock feature in future work. Lastly here, we note that the putative southern and northern bubble shock features are remarkably mirror symmetric (as are the overall $\gamma$-ray bubbles and radio lobes+plumes).

\subsection{Observational Evidence for Condensations Coincident with the Bubbles' Edges}

In Section 1 we described UV absorption evidence (Keeney et al. 2006; Zech et al. 2008) for higher-altitude warm ionized material in the Galactic bulge that is participating in a nuclear fountain flow. We believe that this material is the end state of gas condensing in the bubbles' shell. This warm ionized material seems to be concentrated toward the edges of the bubbles, just as required if the volumetric $\gamma$-ray emissivity is to peak here and as expected for our cooling shell model. Keeney et al. (2006) determine that their clouds are likely launched into a conical outflow that is collimated into a cylinder of radius $\sim 1.6 \mathrm{kpc}$; with this (somewhat uncertain) radius determination they find that their clouds reach maximum heights $z_{\max } \simeq 12 \pm$ $1 \mathrm{kpc}$. Given the qualitative resemblance between this geometry and that of the bubbles, we note that, were the radius used in the Keeney et al. (2006) cloud trajectory calculations rescaled to $3 \mathrm{kpc},{ }^{14}$ the clouds would be inside the bubbles or the radio plumes.

Zech et al. (2008) have measured the metallicity of the fountain material found by them to be significantly supersolar metallicity $\left(\sim 1.6 Z_{\odot}\right)$. It is, thus, a very good prospect for having been launched from the GC. Moreover, the illuminating background UV source is a post-AGB star in the Messier 5 globular cluster at known distance $(7.5 \mathrm{kpc})$ and height $(5.3 \mathrm{kpc})$ above the plane, which, in projection, is coincident with the edge of the northern bubble. It seems more than plausible that the cloud is located in the compression shell (R3) and proffers a lower limit to the radius of the northern bubble

\footnotetext{
14 The original radius determination from Keeney et al. (2006) assumes both that the kinematically distinct clumps of absorbing material they detect along the sightline to Mrk 1383 will rise to the same maximum height and that they are on the front and back intersections of this sightline with the surface of the assumed cylindrical outflow. Relaxing either or both of these assumptions, the radius of the outflow could be different, in general, from $1.5 \mathrm{kpc}$.
}

of $\sim 2.7 \mathrm{kpc}$ (at $z=5.3 \mathrm{kpc}$ ), entirely consistent with the $\sim 3 \mathrm{kpc}$ radius we have adopted.

\subsection{High-speed Entrained Material at the Base of the Northern Bubble}

Fox et al. (2014) have recently reported on further UV absorption observations along the sightline to the quasar PDS $456(l, b)=(10: 4,+11: 2)$, which passes through the conically expanding lower part of the northern bubble at a height of $\sim 2.3 \mathrm{kpc}$. These observations find red- and blueshifted warm ionized material, which kinematic analysis suggests is moving at $\sim 900 \mathrm{~km} \mathrm{~s}^{-1}$, likely accelerated by the ram pressure of the dynamically dominant "wind fluid," i.e., the volume-filling, hot plasma phase. Given this supersonic velocity, this sightline must pass somewhere below the giant shocks. Indeed, the northern shock position we infer from the radio polarization observations discussed in Section 7.1.2 is safely above the sightline. The $\sim 900 \mathrm{~km} \mathrm{~s}^{-1}$ speed inferred by Fox et al. (2014) is somewhat higher than the flow speeds indicated by our model and the heights somewhat above where we predict the shocks to lie on the basis of modeling the global flow parameters. It is tantalizing, however, that this sightline is on the east side of the north bubble, where, observationally, the shock seems to have been pushed furthest from the plane. This could be a spatially and temporarily localized upward excursion in the outflow velocity connected to the recent concentration of star formation activity on the east side of the Galactic center, as already discussed. To obtain a shock $\sim 2.3 \mathrm{kpc}$ height with an upstream flow requires $\dot{E}_{0} / \dot{M} \simeq 5 \times 10^{15} \mathrm{erg} \mathrm{g}^{-1}$ or a mass loading factor $\beta \simeq 1.7$, only a little smaller than the range of $\sim 2-4$ we find for this parameter in the allowed parameter space from our modeling of the global outflow.

\subsection{Tensions with X-Ray Data?}

One possible tension between our model and X-ray observations is the following: modeling of data taken by Suzaku in high-latitude scans across the limbs of both the north and south bubbles (Kataoka et al. 2013) suggests a significantly subsolar metallicity $Z \simeq 0.2 Z_{\odot}$. This is contrary to the simple expectation were this material to be supplied by the nucleus (in which case it would be expected to be at least solar in metallicity). Interestingly, the warm plasma found by Zech et al. (2008) along the Messier 5 sightline, relatively close to the Suzaku northern scan, is supersolar in metallicity, as already remarked. One possible explanation for this apparent tension is that the Suzaku observations predominantly reveal emission from swept-up halo material on the other side of the $\mathrm{CD}$, which various pieces of evidence (as summarized by Miller \& Bregman 2014) suggest has a metallicity of $\sim 0.3 Z_{\odot}$. The northern Suzaku scan does commence significantly outside the edges of the bubbles, within the NPS feature; with six of the eight northern pointings outside the $\gamma$-ray edge and with the metallicity measured only with confidence in the aggregate (and not for individual pointings), a systematic bias toward the metallicity of exterior gas would be expected in our picture that the $\gamma$-ray edge is identified with the CD.

We also note here that the $\sim 300 \mathrm{~km} \mathrm{~s}^{-1}$ expansion speed of the bubbles estimated by Kataoka et al. (2013) is also clearly in tension with our claimed expansion speed of only a $\sim$ few $\mathrm{km} \mathrm{s}^{-1}$. The $300 \mathrm{~km} \mathrm{~s}^{-1}$ estimate, however, is not model independent: it relies on the interpretation that the increase of the 
temperature of the plasma in the vicinity of the bubbles' limbs with respect to the background halo plasma by a factor of $\sim 3 / 2$ can be explained by heating at a weak shock sweeping up the halo gas (which is assumed to be initially in hydrostatic equilibrium). In our model, the plasma inside the CD is heated by the reverse shocks low down in the nuclear outflows into both Galactic hemispheres; we do not specifically deal with the swept-up halo material here (which the Suzaku observations are biased toward, as already mentioned).

Another issue with the X-ray data may be the following: these have been interpreted by Kataoka et al. (2013) using a three-component model incorporating (i) an absorbed powerlaw isotropic background; (ii) a local (unabsorbed) singletemperature, collisional-ionization-equilibrium (CIE) plasma foreground (representing the local plasma bubble); and (iii) an absorbed, single-temperature, CIE plasma that represents the signal region (the NPS and the limb regions in either the north or south bubbles). Such a model may be too simple to adequately describe the putative cooling shell. Indeed, Weaver et al. (2000) and Strickland et al. (2002) demonstrate that fitting to X-ray plasma emission from starburst outflows often leads to unphysically low metallicity estimates. Possible circumstances leading to a too-low metallicity estimate are (i) the existence of neglected multitemperature plasma distributions, (ii) having a large and poorly constrained absorbing column, and/or (iii) nonthermal contribution to the continuum (that boosts apparent free-free emission relative to lines, thus making plasma appear to have smaller metallicity). Any or all of these may be relevant effects for the bubble plasma, in particular, we expect the gas in the cooling shells to be multitemperature, as already discussed, and catastrophic cooling of the plasma into atomic phase may also generate absorbing material.

\subsection{Forward Shock/Shell?}

Mention of halo material swept up ahead of the CD leads us to consideration of the forward shock in our model. In Section 4 we do not consider cooling in the region between the forward shock and the $\mathrm{CD}$. The implication of this is that, with the attainment of pressure balance with the external atmosphere, the forward shock degenerates into a sound wave. Even traveling at only the $\sim 280 \mathrm{~km} \mathrm{~s}^{-1}$ sound speed, however, this would have traveled $\sim 30 \mathrm{kpc} t / 10^{8} \mathrm{yr}$ and, therefore, be currently out of the picture given our estimated age for the bubbles of $\gtrsim$ few $\times 10^{8} \mathrm{yr}$. Yet, observationally, there are, in the northern Galactic hemisphere, very large angular scale $\mathrm{X}$-ray and nonthermal radio and $\gamma$-ray structures that, at least in projection, are external to the bubbles' edges and appear concentric to them. One is tempted to identify these as shock or sound waves associated with the bubbles (e.g., Figure 4 of Su et al. 2010; Figure 1 of Jones et al. 2012.)

In particular, the NPS (Bolton \& Westfold 1950) emerges north from the Galactic plane at $l \sim 20^{\circ}-30^{\circ}$ and rises to heights above $b \sim 80^{\circ}$, merging with the Loop I radio structure (Large et al. 1962; Haslam et al. 1982). It parallels the east edge of the north bubble over this extent; indeed, the NPS and Loop I, together, parallel the edge of the northern polarized radio lobe (which subsumes the north bubble) to a remarkable degree. The NPS exhibits total-intensity and polarized, nonthermal radio, and microwave emission (Sofue \& Reich 1979; Sun et al. 2014; Vidal et al. 2014), thermal X-ray emission (Snowden et al. 1997; Willingale et al. 2003), and $\sim \mathrm{GeV} \gamma$-ray emission (Casandjian et al. 2009; Ackermann et al. 2014).

It has long been argued on the basis of stellar polarization data that the NPS is a structure of the local ISM (within a few $\times 10^{2}$ pc; Bingham 1967; Berkhuijsen et al. 1971). However, a number of recent works have claimed, on the basis of various independent analyses of X-ray, radio polarization, and other data, that it is actually a Galactic-scale feature (Kataoka et al. 2013; Fang \& Jiang 2014; Sun et al. 2014; Sofue 2015; Tahara et al. 2015) or perhaps a confusion of two structures, one local and one at Galactic scales. This finding complements the idea that (some part of) the NPS is a signature of Galactic nuclear activity (Sofue 1977, 2000; Bland-Hawthorn \& Cohen 2003; Totani 2006) and perhaps even related directly to the Fermi bubbles (Guo \& Mathews 2012; Kataoka et al. 2013; Mou et al. 2014). In addition to the NPS, there are linear $\gamma$-ray and polarized radio arcs that run concentrically between the NPS and the eastern edge of the northern bubble (Su et al. 2010; Jones et al. 2012); fainter radio and X-ray spurs have also been claimed as the northwestern, southeastern, and southwestern complements of the NPS (Sofue 2000, 2015; Tahara et al. 2015). We set aside further discussion of this interesting phenomenology for a future publication. We do, however, make the claim here that accounting, in a fuller model, for cooling in the shell of material exterior to the CD implies a different channel for dissipation of the flux of internal energy of swept-up halo gas. This retards the advance of the forward surface of this shell (which, in the absence of cooling, would be the forward shock, degenerating into a sound wave with the attainment of pressure balance), leaving open the possibility that this feature could be identified with the NPS + Loop I feature. Note that the question of why the northeastern feature is so much more conspicuous than its northwest, southeast, or southwest analogs is not addressed in the current speculation.

\section{DISCUSSION AND CONCLUSION}

In this paper we have set out what we believe is essentially the correct framework for understanding the Galactic center's giant outflows. These reveal themselves in three disparate nonthermal phenomena: the $\gamma$-ray Fermi bubbles, the microwave haze, and the polarized "S-PASS" radio lobes. Requiring only that the bubbles contain the expected shocks and assuming that they are expanding into an atmosphere of finite pressure, $\sim 2 \mathrm{eV} \mathrm{cm}^{-3} \simeq 3.2 \times 10^{-12} \mathrm{dyn}^{-2}$, consistent with X-ray and other constraints, we have scanned over the parameter space for the nuclear energy and mass injection rates, $\left\{\dot{E}_{0}, \dot{M}\right\}$. There are very few other adjustable parameters in our model.

1. The effective temperature of the compression shell, $3.5 \times 10^{6} \mathrm{~K}$, is given by $\mathrm{X}$-ray observations.

2. The solid angle of each conical outflow, for which we adopt $\Omega=\pi$ as motivated by the observed geometry.

3 . The metallicity of the outflowing gas, which we have conservatively assumed to be solar but which, plausibly, may be higher. We have shown that the dynamics around the inflation of twice-solar bubbles is not very different from that of solar-metallicity bubbles.

4. The fraction of mechanical energy delivered to the reverse shocks that ends up in cosmic rays, $L_{\mathrm{CR}} / \dot{E} \equiv \epsilon_{\mathrm{CR}}$, we find falls in the range $0.05 \lesssim \epsilon_{\mathrm{CR}} \lesssim$ 0.3 with a preferred value close to $\epsilon_{\mathrm{CR}}=0.15$. These 
values are typical for determinations of the cosmic-ray acceleration efficiency of the Galaxy (expressed as a fraction of the input mechanical power from SNe; Hillas 2005; Strong et al. 2010), in giant, star-formation-driven outflows from other systems (e.g., NGC 253: Zirakashvili \& Völk 2006), and for giant shocks in galaxy clusters (Brüggen et al. 2012; Pinzke et al. 2013).

5. The fraction of cosmic-ray luminosity, in turn, that goes to cosmic-ray electrons, which we expect lies in the range $0.05 \lesssim L_{e} / L_{\mathrm{CR}} \lesssim 0.2$ from consideration of the synchrotron phenomenology (adopting $\epsilon_{\mathrm{CR}}=0.15$ ). The $\gamma$-ray phenomenology indicates an even more restrictive range $L_{e} / L_{\mathrm{CR}} \lesssim 0.1$ lest the region $\lesssim 300 \mathrm{pc}$ downstream of the reverse shocks appear too bright in IC $\gamma$-rays. This range is, again, entirely consistent with experience from modeling nonthermal emission from cluster-scale shocks (Pinzke et al. 2013; Brunetti \& Jones 2014, and references therein).

Other inputs to our modeling are the parameterization of the Galaxy's gravitational potential (Breitschwerdt et al. 1991) and the plasma cooling function (Dopita et al. 2013).

Remarkably, we have found that most of the $\left\{\dot{E}_{0}, \dot{M}\right\}$ parameter space can be excluded by measurements and/or physically motivated constraints. Within the region of parameter space that is not excluded, our model reproduces

1. the $\gamma$-ray luminosity, spectrum, and surface brightness distribution of the bubbles, mostly via hadronic emission, but with a non-negligible IC contribution;

2. the luminosity, spectrum, and extent of the hardspectrum, total-intensity microwave haze;

3. the luminosity, spectrum, and extent of the steepspectrum, polarized $\sim \mathrm{GHz}$ S-PASS lobes;

4. the extent, temperature, and density of the bubbles' plasma;

5. the size of the bubbles without fine-tuning; and

6. the mass flux and initial radius of the nuclear outflow and the plasma number density in the injection zone.

This same, residual region of parameter space presents a compelling fit to a star-formation-driven system with parameters that match the conditions in the nucleus. From our modeling, presented in Section 4, of a radiative bubble expanding into an atmosphere of pressure similar to that in the Galactic halo, for values of $\dot{E}_{0}$ and $\dot{M}$ selected by our reverse shock analysis, the asymptotic radius, temperature, and bolometric cooling luminosity of such a bubble are close to the observed values for the Fermi bubbles.

Our model explains both the hard spectral indices of the CR electron population responsible for the haze synchrotron emission and the CR proton and ion population responsible for the (hadronic) bubble $\gamma$-rays and why, within uncertainties, these are the same at $\gamma \simeq 2.1$ : these populations are both (re)accelerated at the giant shocks, which, our modeling shows, are required to have Mach numbers in the range 6-9 within the allowable $\left\{\dot{E}_{0}, \dot{M}\right\}$ parameter space.

Simultaneously, we explain the steep-spectrum, polarized radio emission that subsumes the bubbles. This has not been adequately addressed in other models: this emission is coming from an aged $\left(\gtrsim 3 \times 10^{7} \mathrm{yr}\right.$ old $)$ and mixed population of electrons that is difficult to explain in models that postulate activity $\sim 1$ Myr ago to explain the bubbles. These electrons survive long enough to travel from the internal shocks out to the edge of the bubbles, where they radiate on the strong and rather regular magnetic field compressed into the cooling shell from the inside, supplemented by secondary electrons created in situ. The phenomenon of mixing of low-energy electrons radiating at $2.3 \mathrm{GHz}$ of different ages implies a rather uniform $F_{\nu} \propto \nu^{-1}$ over the solid angle of the bubbles. Our model also allows, however, that for low-energy electrons that break out of the bubbles a further, distance-dependent steepening is incurred because a systematic flow away from the bubbles reestablishes a one-to-one correspondence between (reset) electron age and distance. This detailed phenomenology is indeed reproduced by the polarized radio lobes, which exhibit a rather constant $F_{\nu} \propto \nu^{-1.1}$ spectrum within the boundaries of the bubbles but show a systematic steepening going from the bubble interior into the radio plumes that extend beyond them (see Figure S4 of the supplementary material of Carretti et al. 2013).

For fiducial parameters, our modeling from Section 4 suggests that the bubbles are at least $\sim 5 \times 10^{8} \mathrm{yr}$ old, very similar to the cooling time of plasma at inferred bubble temperature and number density. The bubbles may be related to whatever Galaxy-scale process led to the formation of the CMZ gas torus, which, at $\sim 3 \times 10^{7} M_{\odot}$, has a very similar $\mathrm{H}_{2}$ mass to the plasma content of the bubbles. Sjouwerman et al. (1998) have claimed, on the basis of counting OH/IR stars in the inner $50 \mathrm{pc}$ around the GC, evidence for a starburst in the $\mathrm{GC} \gtrsim 1 \mathrm{Gyr}$ ago, which may be a credible event to initiate the inflation of the bubbles (though we emphasize that their current nonthermal emission and apparent slow growth can be supported by the current or recent nuclear SFR).

In theory, the bubbles would reach their asymptotic final radius after $\sim 1$ Gyr, but they may not survive this long. Indeed, they may already have begun to blow out, as evidenced by the polarized radio plumes that extend significantly beyond their upper $\gamma$-ray edges (Carretti et al. 2013). This process may be meditated by the Rayleigh-Taylor instability with the buoyant fluid of cosmic rays and hot plasma breaking through the overlying shell of relatively denser shell material at the top of the bubbles. If so, the plumes might be expected to grow at the expansion speed, $v_{\exp } \sim 5 \mathrm{~km} \mathrm{~s}^{-1}$, implying a length scale $t_{\text {cool }} \times v_{\text {exp }} \sim 2.6 \mathrm{kpc}$, which is, indeed, similar to their observed extension.

In any case, the plume phenomenology is consistent with a hadronic origin for the bubbles' $\gamma$-ray emission: in this scenario, although (lower-energy) electrons, lower-density plasma, and magnetic fields can leak out of the bubbles and synchrotron-radiate beyond them, leaking hadrons will not radiate (or only weakly) beyond the cooling shell/CD because of a lack of dense target gas there. (Given the ubiquitous CMB, $\mathrm{TeV}$ electrons would also radiate IC from the plumes were they present there, but, as emphasized, in our model these will be concentrated into a zone much farther down in the bubbles, close to their acceleration site in the giant shocks). Before moving on from this topic, we again flag the possibility that the overall extension of the bubbles/radio lobes in a direction west and away from the plane is essentially due to the motion of the Galaxy through the local group medium toward the group barycenter in the direction of Andromeda.

On the basis of our modeling we predict that there should be significant mass in $\sim 10^{4}-10^{6} \mathrm{~K}$ gas-likely exceeding $10^{7} M_{\odot}$-concentrated toward the bubbles' edges. The cooler and denser phases of this gas will be raining down under gravity. 


\subsection{Future Work}

In subsequent publications we will present or examine in more detail the detailed spectral morphology of the synchrotron emission in the bubbles, the observational evidence for the giant reverse shocks we have inferred, a detailed treatment of the dynamical production of the dense condensations, and a full treatment of the dynamic importance of cooling in the zone forward of the CD and the possibility to identify the NPS with the forward surface of a cooling shell of swept-up halo gas. We will also examine the origin of the $\gamma$-ray spectral downturn at $\sim 100 \mathrm{GeV}$ (Ackermann et al. 2014) and the hardening of the $\gamma$ ray spectrum at high latitudes (Selig et al. 2014; Yang et al. 2014). The former is not explicitly dealt with in our current model but may signify the energy where the bubbles themselves cease to trap cosmic-ray protons effectively; the latter may signify an important role for diffusive transport of cosmic rays in the upper reaches of the bubbles (Yang et al. 2014), which is, again, not accounted for in the current model. Also awaiting a detailed treatment is the dynamical importance of the strong and coherent magnetic field that appears to be wrapped over the surface of the bubble, particularly in stabilizing this surface.

\subsection{Further Speculations}

For fiducial parameters, the giant shocks are capable of accelerating cosmic-ray protons to energies around the knee in the observed cosmic-ray spectrum. Given the very hard spectrum, a total power in these particles that approaches $10^{39} \mathrm{erg} \mathrm{s}^{-1}$, and that the particles are delivered into the halo but not too far above the disk, these shocks are interesting candidates (see Parizot 2014) for the main accelerators of Galactic cosmic rays in this energy range (see Cheng et al. 2012; Lacki 2014; Taylor et al. 2014).

Our model selects a region of the $\left\{\dot{E}_{0}, \dot{M}\right\}$ parameter space implying that, if star formation driven, the outflow is mass loaded at a level $\beta \sim 2-4$, broadly consistent with observations of external, star-formation-driven outflows (Strickland \& Heckman 2009, and references threrein). Further, it is remarkable that our results indicate that the mass efflux in the outflow is $\dot{M} \sim 0.03-0.1 M_{\odot} \mathrm{yr}^{-1}$, rather close to the current rate of nuclear star formation (Crocker 2012; Longmore et al. 2013, and references therein), and in turn close to the long-time-averaged nuclear SFR. ${ }^{15}$ Moreover, on the basis of the semianalytic model presented in Section 4, we can say that the Fermi bubble system is rather delicately poised in terms of allowable values of the ratio $\dot{E}_{0} / \dot{M}$ : systems with $\dot{E}_{0} / \dot{M}$ ratios half those favored by our analysis asymptote to final radii within $\sim 10^{8} \mathrm{yr}$ that are smaller than those observed (and contain plasma that is too cold); systems with twice the favored value for this ratio ( of $\sim 1.6 \times 10^{15} \mathrm{erg} \mathrm{g}^{-1}$ ) do not asymptote to a final radius until $\sim 10^{10} \mathrm{yr}$ (and this radius is $10 \mathrm{kpc}$, significantly larger than the observed bubbles) and spend only a brief time in the "vicinity" of the observed size (implying a return to a "Why now?" fine-tuning); see Figure 4.

These observations suggest the operation of a negative feedback process to maintain the required delicate balance. An obvious mechanism to achieve this-completing the cycle-is

\footnotetext{
15 This can be rather directly and robustly determined from the fact that the total stellar mass of the nuclear bulge (the stellar population coincident with the central molecular zone) is $\sim 10^{9} M_{\odot}$, which has been formed over a timescale $10^{10} \mathrm{yr}$ (Serabyn \& Morris 1996; Figer et al. 2004).
}

that material fountaining back to the plane in the vicinity of the nucleus will trigger further star formation in this region. Star formation that is too vigorous or that produces a too large value of $\dot{E}_{0} / \dot{M}$ in the outflow (which may currently be happening on the east side of the nucleus) will tend to switch itself off because the outflowing plasma takes too long to condense and fall back from the downstream of the bubbles. The matter cycle will not be $100 \%$ efficient, of course, but the system is not closed: some amount of accretion to the nucleus will continue through the plane, and, moreover, interaction and mixing between bubble matter and the surrounding halo gas around the CD may catalyze the condensation of halo gas so that, overall, more gas fountains back to the plane than is lofted upward (Marinacci et al. 2010), some of it low metallicity.

R.M.C. is the recipient of an Australian Research Council Future Fellowship (FT110100108). R.M.C. warmly acknowledges enlightening conversations and/or correspondence with Felix Aharonian, Joss Bland-Hawthorn, Luke Drury, Christoph Federrath, Ed Jenkins, Federico Marinacci, Matt Miller, Christoph Pfrommer, Robin Shelton, Heinz Völk, and Alex Wagner and thanks Doug Finkbeiner for first mentioning to him the possible implications of the Milky Way's motion through the local medium for distorting the bubbles. Ralph Sutherland is particularly thanked for supplying plasma cooling data generated by MAPPINGS IV code prior to public release, Alex Hill for providing information about $\mathrm{H} \alpha$ emission in the southern bubble as observed by the Wisconsin H-Alpha Mapper (WHAM) southern sky survey (Haffner et al. 2010), and Ruizhi Yang for providing processed, preliminary maps of the Fermi-detected emission in the southern bubble. We are grateful to the referee for a considered and helpful report.

\section{APPENDIX \\ MODEL OF A SPHERICAL, RADIATIVE BUBBLE}

In this Appendix we develop our model for a spherical bubble (illustrated in Figure 3) driven by the energy input provided by a star-formation-driven superwind in the Galactic center. In this model we do not assume that the bubble is highly overpressured with respect to the ISM, and we also incorporate cooling. We consider equations for energy, mass, and momentum conservation in that order.

Energy equation. Let $\epsilon_{\mathrm{b}}$ and $p_{\mathrm{b}}=(\gamma-1), \epsilon_{\mathrm{b}}$ be the internal energy density and pressure inside the bubble, where $\gamma=5 / 3$ is the polytropic index. Also, let $\rho_{\mathrm{b}}$ be the bubble density between the reverse shock and the CD (assumed spatially constant), $v$ the internal velocity, and $\dot{E}$ (in ergs s${ }^{-1}$ ) the rate of energy injection into the bubble. The cooling rate of gas with temperature $T$ is $\rho^{2} \Lambda_{\rho}(T)=n^{2} \Lambda[T] \operatorname{ergs~} \mathrm{cm}^{-3} \mathrm{~s}^{-1}$, where $\Lambda_{\rho}(T)=(\mu \mathrm{m})^{-2} \Lambda[T] . \Lambda[T]$ is the cooling function based on total number density $n$ and has been evaluated from the MAPPINGS emission code (Sutherland et al. 2013). In the interior of the bubble there is a reverse shock where the ram pressure of the Galactic center wind is of order the internal pressure of the bubble. The energy equation is derived by integrating the total energy equation over the volume, whose bounding surface comprises the reverse shock and the 
outwardly moving CD. The result is

$$
\begin{aligned}
& \frac{d}{d t} \int_{V}\left(\epsilon_{\mathrm{b}}+\frac{1}{2} \rho v^{2}\right) d^{3} x+\int_{S} p_{\mathrm{b}} u_{i} n_{i} d S \\
& \quad=\dot{E}-\int_{V} \rho^{2} \Lambda_{\rho}(T) d^{3} x .
\end{aligned}
$$

In this equation we make the usual assumption of neglecting the kinetic energy of shocked gas inside the bubble in comparison to the internal energy $\left(\rho v^{2} \ll \epsilon\right)$. Let $R_{\mathrm{b}}$ be the radius of the bubble, with volume $V_{\mathrm{b}}=4 \pi / 3 R_{\mathrm{b}}^{3}$. We ignore the volume of the bubble inside the reverse shock. The energy Equation (9.1) can then be cast as an equation for the bubble pressure:

$$
\frac{d p_{\mathrm{b}}}{d t}=-3 \gamma p_{\mathrm{b}} \frac{1}{R_{\mathrm{b}}} \frac{d R_{\mathrm{b}}}{d t}+\frac{3(\gamma-1)}{4 \pi} \frac{\dot{E}}{R_{\mathrm{b}}^{3}}-(\gamma-1) \rho^{2} \Lambda_{\rho}(T) .
$$

Mass. Let $\dot{M}$ be the rate of injection of mass into the bubble, and let $\Delta \dot{M}$ be the rate of mass dropout associated with cooling. We estimate $\Delta \dot{M}$ by equating the enthalpy flux of hot gas associated with $\Delta \dot{M}$ with the energy loss via cooling. That is,

$$
\Delta \dot{M} \times \frac{5}{2} \frac{k T}{\mu \mathrm{m}}=\rho^{2} \Lambda_{\rho} \times V_{\mathrm{b}}
$$

Hence, the mass equation becomes

$$
\frac{d M_{\mathrm{b}}}{d t}=\dot{M}-\frac{2}{5} \frac{\mu \mathrm{m}}{k T} \rho^{2} \Lambda_{\rho}(T) \times \frac{4 \pi}{3} R_{\mathrm{b}}^{3} .
$$

The bubble density is given by

$$
\rho_{\mathrm{b}}=\frac{3}{4 \pi} \frac{M_{\mathrm{b}}}{R^{3}} .
$$

Momentum. Cooling in the hot, tenuous background medium is slow, so that, in the early stages of expansion, the bubble does not form a thin shell, as in the classic case of an interstellar bubble (Castor et al. 1975; Weaver et al. 1977). We assume that the pressure and velocity in the shocked ambient medium are approximately constant. In this case the expansion of the CD (i.e., the surface of the bubble) and the forward shock are governed by the conditions at the forward shock and matching the pressure of the shocked ISM to the bubble pressure. The resulting equations for the velocity of expansion of the bubble and the velocity of the external shock (radius $R_{\mathrm{s}}$ ) are, respectively,

$$
\begin{gathered}
\frac{d R_{\mathrm{b}}}{d t}=\left(\frac{p_{\mathrm{a}}}{\rho_{\mathrm{a}}}\right)^{1 / 2} \frac{\left(p_{\mathrm{b}}-p_{\mathrm{a}}\right) / p_{\mathrm{a}}}{\sqrt{\left.\gamma+(\gamma+1) p_{\mathrm{b}}-p_{\mathrm{a}}\right) / 2 p_{\mathrm{a}}}} \\
\frac{d R_{\mathrm{s}}}{d t}=\left(\frac{\gamma p_{\mathrm{a}}}{\rho_{\mathrm{a}}}\right)^{1 / 2}\left[1+\frac{\gamma+1}{2 \gamma}\left(\frac{p_{\mathrm{b}}-p_{\mathrm{a}}}{p_{\mathrm{a}}}\right)\right]^{1 / 2} .
\end{gathered}
$$

For a large pressure differential $\Delta p=p_{\mathrm{b}}-p_{\mathrm{a}} \gg p_{\mathrm{a}}$,

$$
\frac{d R_{\mathrm{b}}}{d t} \sim\left(\frac{2}{\gamma+1} \frac{\Delta p}{\rho_{\mathrm{a}}}\right)^{1 / 2}
$$

and

$$
\frac{d R_{\mathrm{s}}}{d t} \sim\left(\frac{\gamma+1}{2} \frac{\Delta p}{\rho_{\mathrm{a}}}\right)^{1 / 2}=\frac{\gamma+1}{2} \frac{d R_{\mathrm{b}}}{d d t} .
$$

When the pressure difference approaches zero,

$$
\frac{d R_{\mathrm{b}}}{d t} \rightarrow 0
$$

and

$$
\frac{d R_{\mathrm{s}}}{d t} \rightarrow\left(\frac{\gamma p_{\mathrm{a}}}{\rho_{\mathrm{a}}}\right)^{1 / 2}
$$

so that as the pressure differential tends to zero, the bubble stalls and the ambient shock propagates away from the bubble as a sound wave.

In developing a numerical solution for an expanding bubble, we integrate Equations (9.2), (9.4)-(9.7) with input parameters mass injection rate, $\dot{M}$, te energy flux, $\dot{E}$, and metallicity, $Z$ (which affects the cooling). The solution starts at a time $t=10^{5} \mathrm{yr}$, with initial values defined by the solution of these equations for a highly overpressured bubble, for which negligible mass loss and cooling has occurred. This solution is defined by

$$
\begin{gathered}
R_{\mathrm{b}}=\left[\frac{125}{264 \pi}\right]^{1 / 5} t^{3 / 5} \\
R_{\mathrm{s}}=\frac{4}{3} R_{\mathrm{b}} \\
p_{\mathrm{b}}=\frac{12}{25}\left[\frac{125}{264 \pi}\right]^{2 / 5} t^{-4 / 5} \\
M=\dot{M} t \\
E_{\mathrm{tot}}=\frac{5}{11} F_{E} t,
\end{gathered}
$$

where $E_{\mathrm{tot}}$ is the total energy within the bubble.

\section{REFERENCES}

Ackermann, M., Albert, A., Atwood, W. B., et al. (Fermi-LAT Collaboration) 2014, ApJ, 793, 64

Ade, P. A. R., Aghanim, N., Arnaud, N., et al. (Planck Collaboration) 2013, A\&A, 554, A139

Almy, R. C., McCammon, D., Digel, S. W., et al. 2000, ApJ, 545, 290

Barkov, M. V., \& Bosch-Ramon, V. 2014, A\&A, 565, A65

Bell, A. R. 1978, MNRAS, 182, 147

Berkhuijsen, E. M., Haslam, C. G. T., \& Salter, C. J. 1971, A\&A, 14, 252

Bingham, R. G. 1967, MNRAS, 137, 157

Bland-Hawthorn, J., \& Cohen, M. 2003, ApJ, 582, 246

Bolton, J. G., \& Westfold, K. C. 1950, AuSRA, 3, 19

Breitschwerdt, D., Dogiel, V. A., \& Völk, H. J. 2002, A\&A, 385, 216

Breitschwerdt, D., McKenzie, J. F., \& Voelk, H. J. 1991, A\&A, 245, 79

Brüggen, M., Bykov, A., Ryu, D., \& Röttgering, H. 2012, SSRv, 166, 187

Brunetti, G., \& Jones, T. W. 2014, IJMPD, 23, 30007

Carretti, E., Crocker, R. M., Staveley-Smith, L., et al. 2013, Natur, 493, 66

Casandjian, J.-M., Grenier, I. \& for the Fermi Large Area Telescope Collaboration 2009, arXiv:0912.3478

Castor, J., McCray, R., \& Weaver, R. 1975, ApJL, 200, L107

Cheng, K.-S., Chernyshov, D. O., Dogiel, V. A., et al. 2011, ApJL, 731, L17

Cheng, K.-S., Chernyshov, D. O., Dogiel, V. A., et al. 2012, ApJ, 746, 116

Cheng, K. S., Chernyshov, D. O., Dogiel, V. A., \& Ko, C. M. 2014b, arXiv:1411.6395v1

Cheng, K. S., Chernyshov, D. O., Dogiel, V. A., \& Ko, C. M. 2015, arXiv:1503.03939 
Cooper, J. L., Bicknell, G. V., Sutherland, R. S., \& Bland-Hawthorn, J. 2008, ApJ, 674, 157

Crocker, R. M. 2012, MNRAS, 423, 3512

Crocker, R. M., \& Aharonian, F. 2011, PhRvL, 106, 101102

Crocker, R. M., Bicknell, G. V., Carretti, E., Hill, A. S., \& Sutherland, R. S. 2014, ApJL, 791, L20

Crocker, R. M., Jones, D. I., Aharonian, F., et al. 2011a, MNRAS, 411, L11

Crocker, R. M., Jones, D. I., Aharonian, F., et al. 2011b, MNRAS, 413, 763

de Boer, W., \& Weber, M. 2014, arXiv:1407.4114

Dobler, G. 2012a, ApJ, 750, 17

Dobler, G. 2012b, ApJL, 760, L8

Dobler, G., \& Finkbeiner, D. P. 2008, ApJ, 680, 1222

Dobler, G., Finkbeiner, D. P., Cholis, I., et al. 2010, ApJ, 717, 825

Dopita, M. A., Sutherland, R. S., Nicholls, D. C., Kewley, L. J., \& Vogt, F. P. A. 2013, ApJS, 208, 10

Everett, J. E., Schiller, Q. G., \& Zweibel, E. G. 2010, ApJ, 711, 13

Everett, J. E., Zweibel, E. G., Benjamin, R. A., et al. 2008, ApJ, 674, 258

Fabian, A. C., Sanders, J. S., Williams, R. J. R., et al. 2011, MNRAS, 417, 172

Fang, T., \& Jiang, X. 2014, ApJL, 785, L24

Figer, D. F., Rich, R. M., Kim, S. S., Morris, M., \& Serabyn, E. 2004, ApJ, 601, 319

Finkbeiner, D. P. 2004, ApJ, 614, 186

Fox, A. J., Bordoloi, R., Savage, B. D., et al. 2014, arXiv:1412.1480

Fujita, Y., Ohira, Y., \& Yamazaki, R. 2013, ApJL, 775, L20

Gold, B., Odegard, N., Weiland, J. L., et al. 2011, ApJS, 192, 15

Guo, F., \& Mathews, W. G. 2012, ApJ, 756, 181

Haffner, L. M., Reynolds, R. J., Madsen, G. J., et al. 2010, ASPC, 438, 388

Haslam, C. G. T., Salter, C. J., Stoffel, H., \& Wilson, W. E. 1982, A\&AS, 47, 1

Hillas, A. M. 2005, JPhG, 31, 95

Hinshaw, G., Weiland, J. L., Hill, R. S., et al. 2009, ApJS, 180, 225

Jones, D. I., Crocker, R. M., Reich, W., et al. 2012, ApJL, 747, L12

Kardashev, N. S. 1962, SvA, 6, 317

Kataoka, J., Tahara, M., Totani, T., et al. 2013, ApJ, 779, 57

Keeney, B. A., Danforth, C. W., Stocke, J. T., et al. 2006, ApJ, 646, 951

Kretschmer, K., Diehl, R., Krause, M., et al. 2013, A\&A, 559, A99

Kroupa, P. 2001, MNRAS, 322, 231

Kulsrud, R., \& Pearce, W. P. 1969, ApJ, 156, 445

Lacki, B. C. 2014, MNRAS, 444, L39

Large, M. I., Quigley, M. J. S., \& Haslam, C. G. T. 1962, MNRAS, 124, 405

Longmore, S. N., Bally, J., Testi, L., et al. 2013, MNRAS, 429, 987

Marinacci, F., Binney, J., Fraternali, F., et al. 2010, MNRAS, 404, 1464

McClure-Griffiths, N. M., Green, J. A., Hill, A. S., et al. 2013, ApJL, 770, L4

Mertsch, P., \& Sarkar, S. 2011, PhRvL, 107, 091101

Miller, M. J., \& Bregman, J. N. 2014, arXiv:1412.3116

Molinari, S., Bally, J., Noriega-Crespo, A., et al. 2011, ApJL, 735, L33

Mori, M. 1997, ApJ, 478, 225

Morris, M., \& Serabyn, E. 1996, ARA\&A, 34, 645

Mou, G., Yuan, F., Bu, D., Sun, M., \& Su, M. 2014, ApJ, 790, 109

Muno, M. P., Baganoff, F. K., Bautz, M. W., et al. 2004, ApJ, 613, 326
Parizot, E. 2014, arXiv:1410.2655

Pinzke, A., Oh, S. P., \& Pfrommer, C. 2013, MNRAS, 435, 1061

Porter, T. A., Moskalenko, I. V., Strong, A. W., Orlando, E., \& Bouchet, L. 2008, ApJ, 682, 400

Reynolds, S. P. 2009, ApJ, 703, 662

Selig, M., Vacca, V., Oppermann, N., \& Enin, T. A. arXiv:1410.4562

Serabyn, E., \& Morris, M. 1996, Natur, 382, 602

Shopbell, P. L., \& Bland-Hawthorn, J. 1998, ApJ, 493, 129

Sjouwerman, L. O., Habing, H. J., van Langevelde, H. J., Lindqvist, M., \& Winnberg, A. 1998, in Proc. IAU Symp. 184, The Central Regions of the Galaxy and Galaxies, ed. Y. Sofue (Dordrecht: Kluwer), 129

Skilling, J. 1971, ApJ, 170, 265

Snowden, S. L., Egger, R., Freyberg, M. J., et al. 1997, ApJ, 485, 125

Sofue, Y. 1977, A\&A, 60, 327

Sofue, Y. 2000, ApJ, 540, 224

Sofue, Y. 2003, PASJ, 55, 445

Sofue, Y. 2015, MNRAS, 447, 3824

Sofue, Y., \& Reich, W. 1979, A\&AS, 38, 251

Sofue, Y., Reich, W., \& Reich, P. 1989, ApJL, 341, L47

Stocke, J. T., Keeney, B. A., Danforth, C. W., et al. 2013, ApJ, 763, 148

Strickland, D. K., \& Heckman, T. M. 2009, ApJ, 697, 2030

Strickland, D. K., Heckman, T. M., Weaver, K. A., Hoopes, C. G., \& Dahlem, M. 2002, ApJ, 568, 689

Strong, A. W., Porter, T. A., Digel, S. W., et al. 2010, ApJL, 722, L58

Su, M., \& Finkbeiner, D. P. 2012, ApJ, 753, 61

Su, M., Slatyer, T. R., \& Finkbeiner, D. P. 2010, ApJ, 724, 1044

Sun, X. H., Gaensler, B. M., Carretti, E., et al. 2014, MNRAS, 437, 2936

Sutherland, R., Dopita, M., Binette, L., \& Groves, B. 2013, Astrophysics Source Code Library, record ascl: 1306.008

Tahara, M., Kataoka, J., Takeuchi, Y., et al. 2015, arXiv:1501.04405

Taylor, A. M., Gabici, S., \& Aharonian, F. 2014, PhRvD, 89, 103003

Thoudam, S. 2013, ApJL, 778, L20

Totani, T. 2006, PASJ, 58, 965

van der Marel, R. P., Besla, G., Cox, T. J., Sohn, S. T., \& Anderson, J. 2012, ApJ, 753, 9

Vidal, M., Dickinson, R., \& Leahy, J. 2014, arXiv:1410.4438

Wardle, M., \& Yusef-Zadeh, F. 2014, ApJL, 787, L14

Weaver, K. A., Heckman, T. M., \& Dahlem, M. 2000, ApJ, 534, 684

Weaver, R., McCray, R., Castor, J., Shapiro, P., \& Moore, R. 1977, ApJ, 218,377

Willingale, R., Hands, A. D. P., Warwick, R. S., Snowden, S. L., \& Burrows, D. N. 2003, MNRAS, 343, 995

Yang, H.-Y. K., Ruszkowski, M., Ricker, P. M., et al. 2012, ApJ, 761, 185

Yang, R.-Z., Aharonian, F., \& Crocker, R. 2014, A\&A, 567, A19

Zech, W. F., Lehner, N., Howk, J. C., et al. 2008, ApJ, 679, 460

Zirakashvili, V. N., \& Völk, H. J. 2006, ApJ, 636, 140

Zubovas, K., King, A. R., \& Nayakshin, S. 2011, MNRAS, 415, 21

Zubovas, K., \& Nayakshin, S. 2012, MNRAS, 424, 666 